\title{
ANÁLISE INTRABLOCOS DE EXPERIMENTOS EMPARCELAS SUBDIVIDIDAS COM TRATAMENTOS PRINCIPAIS EM BLOCOS INCOMPLETOS PARCIALMENTE BALANCEADOS
}

\author{
AUGUSTO RAMALHO DE MORAIS \\ Engenheiro Agrônomo
}

Orientador: Prof:. Dr: MARIA CRISTINA STOLF NOGUEIRA

\begin{abstract}
Tese apresentada aे Escola Superior de Agricultura "Luiz de Queiroz" da Universidade de São Paulo, para obtençãa do título de Doutor em Agronomia, Area de concentraça: Estatística e Experimentaçăo Agronômica.
\end{abstract}

\author{
$P$ I $R A C I C A B A$ \\ Estado de Säo Paulo - Brasil \\ Agosto - 1992
}


Ficha catalografica preparada pela serão de Livros da Divisao de Biblioteca e Documentasão - PCAP/USP

\section{Morais, Augusto Ramalho de}

M827a Análise intrablocos de experimentos em parcelas subdivididas con tratamentos prineipaín em blocos incompletos parcialmente balarceados. Piracicaba, 1992. $115 \mathrm{p}$.

Tese - ESALQ

Bibliografia.

1. Analise de varianoia 2. Delineanento experimento 3. Estatistica experimental I. Escola Supexior de Adricultura Luiz de Gueiroz, Piracicaba.

CDD 519.535 
ANALISE INTRABLOCOS DE EXPERIMENTOS EM PARCELAS SUBDIVIDIDAS COM TRATAMENTOS PRINCIPAIS EM BLOCOS INCOMPLETOS

PARCIALMENTE BALANCEADOS

AUGUSTO RAMALHO DE MORAIS

Aprovada em: 14.09 .1992

Comissão julgadora:

Prof. Dr. Maria Cristina Stolf Nogueira

ESALQ/USP

Prof. Dr. Humberto de Campos

ESALQ/USP

Prof. Dr. Antonio Francisco Iemma

ESALQ/USP

Prof. Dr. Gilnei de Souza Duarte

ESAL

Prof. Dr. Sergio do Nascimento Kronka

FCAJ/UNESP

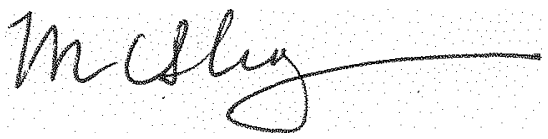

Prof. Dr. MARIA CRISTINA STOLF NOGUEIRA

Orientador 
A minha mäe Maria Aparecida, a meu pai Cipriano (in momoriam) A meus avos (Joaquim, Adalgiza, Manoel) in memorian e Umbelina

\section{OEEREGO}

Ao Grupo Espirita Vovo Christiano, pela forca interior

A verdadeira amizade e eterna, e aumenta a cada dia, apesar das distancias.

A minha esposa. Icléa,

As minhas filhas: Cristiane e Elisa 


\section{AGRADECIMENTOS}

\section{A VIDA}

A Dra. Maria Cristina Stole Nogueira, professora do Departamento de Matemática e Estatistica (DME) pela valiosa orientação e amizade.

Aos professores Antonio F. Iemma, Cássio R. M. Godoi, Clarice G.B. Demetrio, Decio Barbin, Humberto de Campos e Rodolfo Hoffmann pela receptividade e ensinamentos ministrados.

A Escola Superior de Agricultura "Luiz de Queiroz" (ESALQ) da USP e ao Centro Nacional de Pesquisa de Mitho e Sorgo da EMBRAPA pela oportunidade concedida para a realização do curso.

Ao Conselho Nacional de Desenvolvimento Científco e Tecnologico (CNPq) pela bolsa concedida.

A Escola Superior de Agricultura de Lavras na pessoa do Prof. Gilnei de Souza Duarte que nos iniciou nos estudos, pelo desenvolvimento e continuaça do trabalho.

Aos colegas de curso Paulo Cecon, Amauri Machado, Creusa, Maria Cecllia, Antoni Guidoni, Sônia e Luiz Eloj (in memoriam, Joel, Rui e Eufrazio pela amizade.

Aos funcionários do DME da ESALQ/USE pela amizade e cooperação.

$\lambda$ minha esposa Iclea e minhas filhas Cristiane e Elisa pelo companheirismo, compreensão e incentivo no curso e na vida.

Aqueles que, mesmo sem eu o saber, auxilizram para a realizaça do curso. 
iv.

SUMARIO

Página

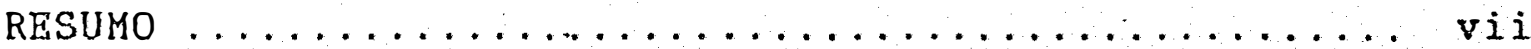

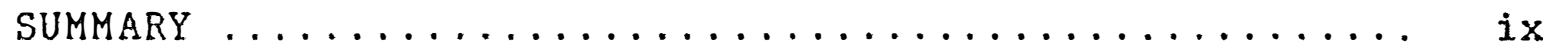

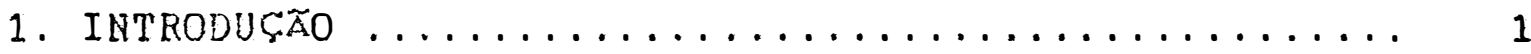

2. REVISÃO DE LITERATURA ..................

2.1. Experimentos em parcelas subdivididas ........ 4

2.1.1. Caracterizaça ................ 4

2.1.2. Analise de Variancia .............. 5

2.1.3. Estimabilidade ................ 6

2.2. Experimentos em Blocos incompletos parcialmente balanceados $(\mathrm{PBIB}) \ldots \ldots \ldots \ldots \ldots \ldots$

2.2.1. Caracterização e definiçăo ........... 9

2.3. PBIB com duas classes de associados - PBIB(2) . 11

2.3.1. Caracterizaça ................ 11

2.3.2. Classificaçăo ................ 13

2.3.3. Tipo Érupo divisıvel ............ 13

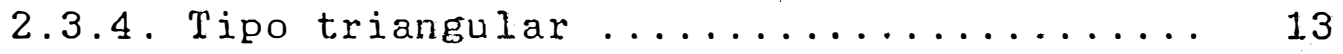

2.3.5. Tipo guadrado latino .............. 14

2.3.6. Geometria paroial ( $r ; k, t) \ldots \ldots 15$

2.4. Experimentos en reticulados quadrados ........ 16

2.4.1. Caracterização ................ 16

2.4.2. Classiticaç̃o ............... 17

2.5. Anslise da Variancia intrablocos ........... 17

2.6. Estimabilidade ................... 21

2.7. Experimentos de parcelas subdivididas em blocos

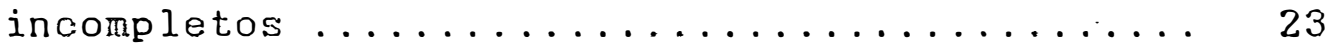

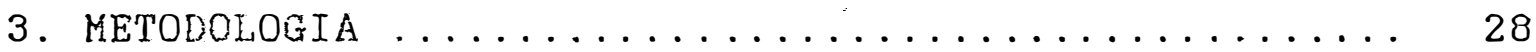

3.1. Caracterizaçăo ................ 28

3.2. Un modelo lineax .................. 29

3.3: Sistema de equações normais ............. 31

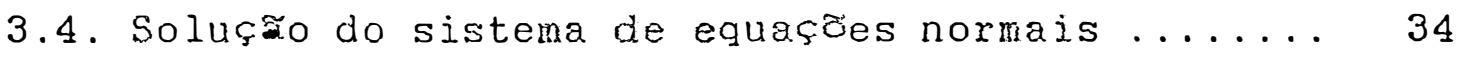

3.4.1. PBIB com m classes de associados ...... 35

3.4.1.1. Estjmaçăo dos efeitos de tratamentos principais ........ 36 
Página

3.4.1.2. Estimaço dos efeitos de tratamentos secundários ........ 43

3.4.1.3. Estimacão dos efeitos de interaça ................. 44

3.4.2. PBIB com duas classes de associados ... 46

3.4.3. Caso especial: Experimentos em blocos .. incompletos balanceados ............ 49

3.4.4. Experimentos reticulados quadrados .... 50

3.5. Matriz de variancias e covariancias ......... 52

3.5.1. Teste de homogeneidade ........... 53

3.5.2. Teste de uniformidade ............ 54

3.6. Propriedades das soluçðes .............. 55

3.6.1. Esperança matemática de $\hat{\tau} \ldots \ldots \ldots \ldots \ldots 55$

3.6.2. Matriz de dispersão para os tratamentos principais ................. 55

3.6.3. Esperança matemática de $\hat{\tau}^{*} \ldots \ldots \ldots \ldots . . \ldots 6$

3.6.4. Matriz de dispersão para os tratamentos secundários .................. 57

3.6.5. Esperanca matemática da interaça $\hat{\delta} \ldots . \quad 57$

3.6.6. Matriz de dispersăo para a interaçăo ... 58

3.7. Análise da variânoia $\ldots \ldots \ldots \ldots \ldots \ldots \ldots \ldots \ldots 61$

3.7.1. Somas de quadrados .............. 61

3.7.2. Soma de quadrados de resíduo ......... 63

3.7.3. Esperança matemática das somas de guadrados ..................... 65

3.7.3.1. Esperança matemática de SQC ... 65

3.7.3.2. Esperança matemática de $\operatorname{SQT}(a j) \quad 66$

3.7.3.3. Esperança matemática de SQB ... 67

3.7.3.4. Esperança matemática de $\mathrm{SQT}^{*} \ldots 68$

3.7.3.5. Esperança matemática de SQI ... 69

3.7.3.6. Esperança matemática de SQTot. 70

3.7.3.7. Esperança matemática de SQPar. 71

3.7.3.8. Esperança matemática de $\operatorname{SQR}(a) \quad 71$

3.7.3.9. Eenerence Matematica de SQR (h) 7 ? 
Página

3.7.4. Decomposiçãa da soma de quadrados de

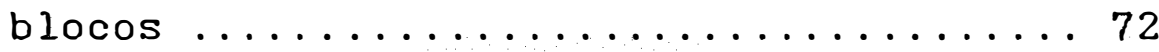

3.8. Distribuiça e independência das formas

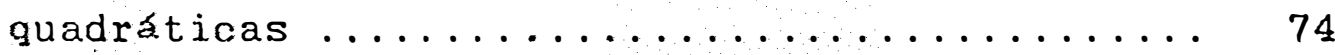

3.9. Esquema de análise da variancia .......... 77

3.10. Estimabilidade .................. 77

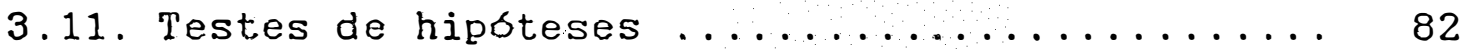

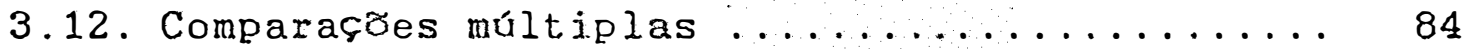

3.12 .1 . Um contraste entre duas médias de tratamentos principais ........... 85

3.12.2. Um contraste entre duas médias de

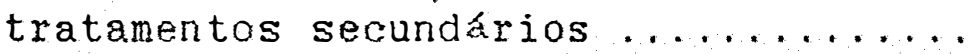

3.12.3. Um contraste entre duas medias de tratamentos secundários, dado um trata-

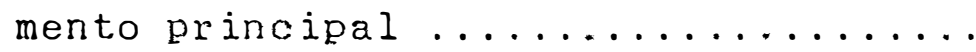

3.12.4. Um contraste entre duas medias de tratamentos principais, dado um tratamen-

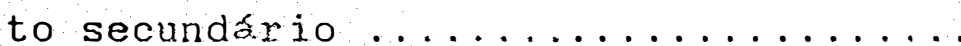

3.12.5. Um contraste entre dois efejtos estimados de tratamentos secundários, dado um tratamento principal .......... 88

3.12.6. Um contraste entre dois efeitos estimados de tratamentos principais, dado um tratamento secundário ........ 89

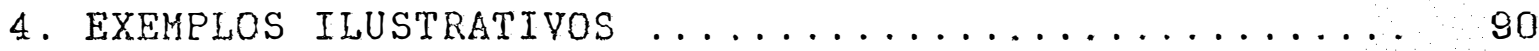

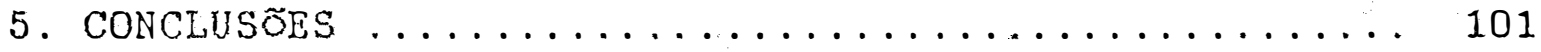

6. REFERENCIAS BIBIIOGRAEICAS ............... 103

7. APENDICE 1. Núcleos das formas quadraticas ....... 110 APENDICE 2. Um programa de análise ............ 114 
vii.

\title{
ANALISE INTRABLOCOS DE EXPERIMENTOS EM PARCELAS SUBDIVIDIDAS COM TRATAMENTOS PRINCIPAIS EM BLOCOS INCOMPLETOS PARCIALMENTE BALANCEADOS
}

\author{
Autor: AUGUSTO. RAMALHO DE MORAIS \\ Orientador: PROF ${ }^{a}$ DR. MARIA CRISTINA STOLE NOGUEIRA
}

RESUMO

O objetivo deste trabalho foi desenvolver uma metodologia adequada para análise de experimentos em parcelas subdivididas com tratamentos principais dispostos segundo uma estrutura de blocos incompletos parcialmente balanceados (PBIB), caracterizados pelos seguintes parámetros: $v$ tratamentos principais; b blocos; $r$ repetisees de cada tratamento principal; k parcelas em cada bloco; u tratamentos secundários, e ainda, $\lambda_{1}, \ldots, \lambda_{m}, n_{\star}, \ldots, n_{m}$, $p_{j k}^{i}(i, j, k=1, \ldots, m)$ delinidos de acordo com BOSE \& NAIR (1939).

$$
\begin{aligned}
& \text { Para esse fim, adotou-se o modelo matenático: } \\
& y_{i j l}=\mu+b_{j}+t_{i}+t_{i}+\delta_{i l}+\delta_{i j l}
\end{aligned}
$$

onde, $\operatorname{para} i=1,2, \ldots, v ; j=1,2, \ldots, b ; 1=1,2, \ldots, u$;

$y_{i j l}$ é a observacão correspondente ao i-ésimo tratamento principal com o l-esimo tratamento secundário no j-ésimo bloco;

He uma constante que representa a media seral;

$t_{i} \in$ o efeito do $i$-esimo tratamento principal;

$b_{j}$ é o efeito do j-ésimo bloco;

$t_{\imath}^{*}$ é o efeito do l-ésimo tratamento secundário;

$\delta_{i l} \in$ o efeito da interaça entre o i-ésimo tratamento principal e o 1-ésimo tratamento secundário;

${ }_{i j l}$ é o erro experimental associado à observaça $y_{i j l}$, normaimente distibuicu, lal que 
viii.

$$
\begin{aligned}
& E\left(\overline{8}_{i, j l}\right)=0 \\
& \operatorname{Cov}\left(\mathscr{s}_{i j L} ; \alpha_{i j l}\right)= \begin{cases}\sigma_{a}^{2}+\alpha_{b}^{2}, & \text { se } i=i, j=j, 1=1 \\
\sigma_{\alpha}^{2}, & , \text { se } i=i, j=j, 1 \neq 1 \\
0, & , \text { caso contrário }\end{cases}
\end{aligned}
$$

Para maior facilidade nas deducoses teoricas, utilizou-se o modelo na forma matricial y $=x+\varepsilon$, sendo y um vetor de observaçes; $X$ a matriz do delineamento; $\theta$ um vetor de parametros e $\varepsilon$ um vetor de variaveis aleatorias não observaveis, assumidas serem normalmente distrituidas com $\varepsilon \sim R(\Phi, \Sigma)$.

Utilizando-se o metodo dos minimos quadrados, sob as condiçes anteriores, foram determinados: o sistema de equaçชes normais; as soluçতes para os efeitos ajustados de tratamentos principais, tratamentos secundarios e interasão; as matrizes de dispersão para os efeitos los parametros; somas de quadrados e suas esperancas matenáticas; as distribuiçes das formas quadraticas; variancias para contrastes entre medias de tratamentos principais, tratamentos secundarios e interacão e funç্s estiraveis.

Algumas das conclustes abtidas foram:

a) As expressos dos estimadores dos efeitos de tratamentos principais obtidas, foram analogas aquelas encontradas para os experimentos em blocos incompletos balanceados e, as expresşes referentes aos tratamentos secundarios e a interafão foram semelhantes àquelas correspondentes nos experimentos em parcelas subdivididas em blocos casualizados.

b) As funcoes estimaveis de tratamentos principais dado um tratamento secundário foram obtidas a partir de combinaç̧es lineares das observasoes presentes no mesmo bloco, sendo algumas não estimáveis.

Exemplos numericos foram apresentados para ilustrasão da metodologia proposta. 


\title{
INTRABLOCF ANALYSIS OF SPLIT-PLOT EXPERIMENTS WITH MAIN TREATMENTS IN PARTIALLY BALANCED INCOMPLETE BLOCKS
}

\author{
Author: AUGUSTO RAMALHO DE MORAIS \\ Adviser: PROF. DR. MARIA CRISTINA STOLF NOGUEIRA
}

SUMMARY

The objetive of this study was to develop one suitable methodology for the intrablock analysis of experiments in split-plot involving a two-way treatment structure when the design structure for whole plot experimental units is a partially balanced incomplete block design. This experiment is characterized by the parameters: $\mathrm{v}$ is the number of main treatment (treatment whole plots); b is the number of blocks; $r$ is the number of replications; $k$ is the number of plot per blcck; $u$ is the number of secondary treatment (treatment of the subplots) and $\lambda_{1}, \ldots$, $\lambda_{m}, n_{1}, \ldots, n_{m}, p_{j k}^{i}(i, j, k=1, \ldots, m)$ defined according to BOSE and NAIR (1939).

For this, the following mathematical model was considered:

$$
y_{i j l}=\mu+b_{j}+t_{i}+t_{i}^{*}+\delta_{i l}+\delta_{i j l}
$$

where, for $i=1,2, \ldots, v ; j=1,2, \ldots, b ; 1=1,2, \ldots, u$;

$y_{i j l}$ denote the observation of the subplot containing the 1 -th secondary treatment of the $i$-th main treatment in the $j$-th block;

$\mu$ denote the effect of the constant general mean;

$t_{i}$ denote the $i-t h$ main treatment effect;

$b_{j}$ denote the $j-t h$ block effect;

$t_{i}$ denote the $1-t h$ secondary treatment effect;

$\delta_{i l}$ denote the interaction between $i-t h$ main treatment and 1-th secondary treatment effect; 
$*_{i j l}$ denote the error inherent to the ijl th subplot, with $E\left(8_{i j l}\right)=0$

$$
\operatorname{Cov}\left(\varangle_{i j l}, \varangle_{i j j^{\prime}}\right)= \begin{cases}\sigma_{a}^{2}+\sigma_{b}^{2}, & \text { if } i=i^{\prime}, j=j^{\prime}, 1=1 \\ \sigma_{a}^{2} & , \text { if } i=i^{\prime}, j=j^{\prime}, 1 \times I^{\prime} \\ 0 & , \text { otherwise }\end{cases}
$$

The linear model used can be expressed as

$\mathrm{y}=\mathrm{X} \theta+\varepsilon$, where $\mathrm{y}$ is an vru-component vetor of observations; $X$ a real matrix (of order vru $x$ p) of known coefficients; $\theta$ a vetor of the parameters unknown and $\varepsilon$ is a vetor of non-observable random variables also termed as error component, which is assumed to be normally distributed as $\varepsilon \sim N(\Phi, \Sigma)$.

Using the least square procedure under such conditions, in order to determine: the system of normal equations; the solutions of the parameters effects; their dispersion matrix for treatment effects; the sum of squares of the parameters; their mathematical expectations; the distributions of the quadratic forms; as well as the justification of the $F$ test, and the variances of the contrasts among means of treatments.

Some of the conclusions obtained were:

i) The estimates of main treatments and blocks effects were analogous to those existent in the literature of the balanced incomplete block; while the estimates of sub treatments and interactions were analogous to those correspondents in the split-plot randomized block design.

ii) The estimable linear functions of the main treatments effects in the same sub treatment were obtained through the linear combinations of the observations present in the same block.

$$
\text { Numerical examples were presented to }
$$
illustrate the proposed methodology. 


\section{INTRODUC̆ÃO}

Un dos problemas fundamentais ao se planejar um experimento, está na escolha correta do tipo de delineamento que melhor se adapte as condiços experimentais especificas do problema em estudo. Na experimentaça, quando se têm dois ou mais fatores para serem estudados simultaneamente, uma das opçres \& a utilizaça do experimento em parcela subdividida.

o experimento em parcela subdividida envolve uma estrutura de tratamentos, con dois ou mais fatores, com uma estrutura de delineamento em blocos incompletos, apresentando, pelo menos, dois tamanhos diferentes de unidades experimentais: as unidades experimentais grandes, chamadas de parcelas principais ou parcelas e as unidades menoces, chamadas de subparcelas. Nas parcelas são casualizados os tratamentos primários e nas subparcelas os tratamentos secundários, segundo MILLIKEN \& JOHNSON (1984).

Esse tipo de experimento e largamente utilizado nas pesquisas agricola, industrial e biologica, mas pode ser usado em outras áreas. Os experimentos. em parcelas subdivididas são úteis em situaç̋es, tais como: a) quando os niveis de um ou mais fatores exigem grandes quantidades do material experimental (por exemplo, métodos de preparo do solo); b) quando informaçতes prévias asseguram que as diferenças entre os niveis de un dos fatores são maiores do que as do outro fator; c) quando se deseja maior precisão para comparaços entre niveis de um dos fatores; d) quando existe um fator de maior importancia e outro de importancia secundária, sendo que este é inciulic pala 
aumentar a extensao dos resultados e e) nas situaçes práticas onde $\&$ dificil a instalacão do experimento no esquema fatorial.

O experimento em parcela subdividida proposto por F. Yates, vem tendo uma grande utilidade para pesquisadores das mais diversas áreas de pesquisa. Uma farta bibliografia sobre o assunto, sua aplicaço e a metodologia de análise está ressaltada em diversos livros textos, tais como, LEONARD \& CIARK (1939), REMPTHORNE (1952), COCHRAN \& COX (1957), STEEL \& TORRIE (1960), HICKS (1973) e PIMENTEL GOMES (1985), dentre outros.

Segundo IEMMA (1981), existe uma lacuna quanto a experimentos em parcelas subdivididas em blocos incompletos. O autor, considerou o caso de experimentos em parcelas subdivididas quando os tratamentos principais estão dispostos na estrutura de delineamento em blocos incompletos balanceados (BIB).

Entretanto, existem situaçres em que o delineamento BIB, nem sempre é viável, pois pode exigir um número elevado de repetiços, tornando-se praticamente impossivel sua utilização.

0 presente trabalho tem como objetivo apresentar uma analise intrablocos, para experimentos em parcelas subdivididas quando os tratamentos principais estão dispostos segundo uma estrutura de delineamento em blocos incompletos parcialmente balanceados, no que se refere a: a) obtenção de soluçes para os efeitos de tratamentos; b) variancias para contrastes entre os efeitos de tratamentos; c) dedução das formulas das somas de quadrados que comprem a análise da variancia e a composicão do teste $F$. 


\section{REVISAOO DE LITERATURA}

Um delineamento experimental é composto de duas estruturas básicas: a) uma "estrutura de tratamentos" que consiste de um conjunto de tratamentos, combinacres de tratamentos ou de populaçes que o pesquisador deseja estudar ou comparar; b) uma "estrutura de delineamento" que consiste no agrupamento das unidades experimentais em grupos homogêneos ou blocos.

A estrutura de delineamento de um experimento e obtida pelo tipo de agrupamento das unidades experimentais em grupos tao uniforme quanto possivel, de acordo com MILLIKEN \& JOHNSON (1984). Se todas as unidades experimentais sao muito homogeneas, entao existe somente um grupo ou bloco de observaçres, e as unidades experimentais podem ser consignadas para os tratamentos completamente ao acaso. Tal estrutura de delineamento é chamada de delineamento inteiramente casualizado.

Se mais do que um grupo de unidades experimentais equerido, de modo que as unidades dentro de um grupo sao muito mais homogeneas que as unidades experimentais entre grupos, entao a estrutura de delineamento é de um delineamento em blocos casualizados. Assim, um delineamento experimental envolve: i) a escolha da estrutura de tratamentos, $i i)$ a escolha da estrutura de delineamento e iii) o método de casualização. Uma estrutura de delineamento em blocos incompletos ocorre quando o número de tratamentos excede o número de unidades experimentais no bloco, assim como, um conjunto completo de tratamentos nao ocorre dentro de cada bioco. 
4.

Neste trabalho, ao se referir a experimentos en parcelas subdivididas estara se reportando a um delineamento experimental com uma estrutura de tratamentos com dois fatores e uma estrutura de delineamento em blocos incompletos caracterizado por dois tamanhos diferentes das unidades experimentais.

\subsection{Experimentos en parcelas subdivididas}

\subsubsection{Caracterizaça}

o experimento em parcela subdividida teve seu inficio com $F$. Yates, por volta de 1933 , segundo LEONARD \& CIARE (1939).

0 experimento em parcela subdividida e constituido a partir de uma estrutura de delineamento em blocos incompletos e uma estrutura de tratamento envolvendo dois ou mais fatores ou tipos de tratamentos. Assin, a combinaço de estrutura de delineamento e estrutura de tratamento para o experimento em parcela subdividida gera diferentes tamanhos de unidades experimentais.

Os experimentos em parcelas subdivididas tem sido utilizados principalmente na analise de dados originados de pesquisas agrlcolas, industriais e biologicas. Este procedimento está apresentado e discutido em vários livros textos, tais como, KEMPTHORNE (1952), STEEL \& TORRIE (1960), COCHRAN \& COX (1957), PIMENTEL GOMES ( 1985$)$ e DAS \& GIRI (1986); em trabalhos de dissertasão e tese, tais como, CONDE (1974), PINHO (1975) e SANCHES (1980), dentre outros. Eles se caracterizam cono sendo uma variaçăo do experimento fatorial com dois fatores, onde os tratamentos principais ( T) das parcelas sao dispostos segundo um tipo qualquer de delineamento experimental, como: blocos casualizados, quadrado latino etc, e os tratamentos secundários (T') das subparcelas săo dispostos, aleatoriamente, dentro de cada parcela.

PIMENTEL GOMES (1985), aconselhou o uso de experimentos em rarrelas subdivididas em alguns dos casos em 
que se estudam dois tipos diferentes de tratamentos. Dentre suas múltiplas aplicaçres, destaca como exemplo, variedades e espaçamentos. Nesse caso, cada parcela contendo um certo espaçamento, é repartida em subparcelas, cada uma contendo uma das variedades.

De acordo com CAMPOS (1984), existem dois tipos de experimentos, no tocante a estruturaça das subparcelas: a) parcelas subdivididas no esparo, quando em cada parcela há uma subdivisão em diversas partes, cada uma delas constituindo as subparcelas, que sera casualizadas dentro de cada parcela e b) parcelas subdivididas no tempo, quando as subparcelas são constitusdas pelas amostras tomadas periodicamente em cada parcela, sendo cada anostra uma subparcela.

\subsubsection{Analise da variancia}

A analise de um experimento em parcelas subdivididas foi discutia por vários autores, entre outros, FEDERER (1955), HARTER (1981), CAMPOS (1984) e NOGUEIRA (1991), os quais, apresentaram exemplos práticos do uso desses experiments nos delineamentos: inteiramente ao acaso e em blocos casualizados, considerando independencia entre os erros a nivel de subparcelas e de parcelas.

$\mathrm{Na}$ análise estatistica de parcelas subdivididas, considera-se, tamben, o fato de que, as observaçzes efetuadas em diferentes subparcelas pertencentes a um dado tratamento prinoipal, são esperadas que sejam correlacionadas, de acordo com CHARRABARTI (1962). E, conforme LEAL (1979), supze-se, que entre os erros experimentais, a nivel de subparcelas, do tipo $e_{i j s}$ e $e_{i j s}$, para quaisquer duas subparcelas distintas (na mesma parcela), existe uma correlação constante $\rho$, e que os erros entre subparcelas em diferentes parcelas não são correlacionados.

De acordo com CHARRABARTI (1962), COCHRAN \& COX (1957) e LEAL (1979), para esses experimentos tem-se:

$$
\underline{E}\left(e_{i j s}\right)=0, \quad \operatorname{Vax}\left(a_{i j s}\right)=\operatorname{Var}\left(y_{i j s}\right)=\sigma^{2}
$$




$$
\operatorname{Cov}\left(e_{i j \varepsilon^{\prime}} e_{i j^{\prime},}\right)=\left\{\begin{array}{c}
\rho \sigma^{2}, \quad \text { para } i=i, j=j, \quad s x^{\circ} \\
0, \quad \text { para os outros casos }
\end{array}\right.
$$

Consideraçzes práticas sobre os experimentos em parcelas subdivididas foram apresentadas por COCHRAN \& COX (1957) e FEDERER (1955), que mostraram ser vantajoso o seu uso, se: a) os efeitos dos tratamentos aplicados às subparcelas ( $T^{\prime}$ ) e os de interação ( $T \times T^{\prime}$ ) são de maior interesse que os efeitos de tratamentos aplicados as parcelas ( $T$ ) e b) os efeitos de $\mathrm{T}$ são medidos com grandes quantidades de material. E mostraram como desvantagens: a) às vezes o erro das parcelas é medido com menor precisão do que o erro de subparcelas e b) as diferentes comparaças entre tratamentos possuem variancias distintas.

0 uso de experimentos em parcelas subdivididas foi abordado por LEAL (1979), como uma alternativa, na análise de experimentos com medidas repetidas, no caso em que as medidas tomadas em intervalos distintos tem variancia homogenea e correlaçes constantes, ou seja, a matriz de variancias e covariancis e do tipo uniforme.

Essa pressuposiç̃o está en acordo com STEEL \& TORRIE (1960) e LITTLE \& HILLS (1978), os quais consideraxam que experimentos com observaçes medidas sucessivamente, sob a mesma unidade experimental, durante um certo intervalo de tempo, se assemelham em varios aspectos com experimentos em parcelas subdivididas, nos quais cada unidade e dividida em sub-unidades experimentais distintas.

REGAZZI (1984), abordou o caso dos experimentos com parcelas subdivididas em blocos casualizados, com tratamentos secundarios ocorrendo em apenas alguns dos tratamentos principais.

\subsubsection{Estimabilidade}

No tocante às funças lineares estináveis, yuatro dasos para comparasas do modias de tratementos form 
7.

considerados por FEDERER (1955), LITTLE \& HILLS (1978) e PIMENTEL GOMES (1985), e podem ser resumidos no sequinte quadro:

Comparaça entre duas médias

dos efeitos estimados entre
Estimativas das variancias

Tratamentos nas parcelas ( $T$ )

$2 \mathrm{E}_{\mathrm{a}} / \mathrm{KR}$

Tratamentos nas subparcelas ( $T^{\prime}$ )

$2 E_{b} / I R$

Tratamentos $T$ ' num mesmo nivel de $T$

$2 E_{b} / R$

Tratamentos $T$ num mesmo nivel de $T$.

$2\left[E_{a}+(K-1) E_{b}\right] / K R$

onde: $I$ = número de tratamentos primários;

$\mathrm{K}=$ número de tratamentos secundários;

$\mathrm{R}=$ número de repetiçßes;

$E_{a}=$ QHikesiduo(a), err a nível de parcelas;

$E_{b}=$ QMResiduo(b), erro a nivel de subparcelas.

ANDERSON \& BANCROFT (1952), COCHRAN \& COX

(1957) e STEEL \& TORRIE (1960), incluiram, ainda, um contras- te para comparar duas medias dos tratamentos ( $T$ ) em diferen-tes niveis de 'T'.

Em CHARRABARTI (1962), encontrou-se o seguinte esquema para algumas funçes lineares nos parametros, seus estimadores e suas respectivas variancias: 


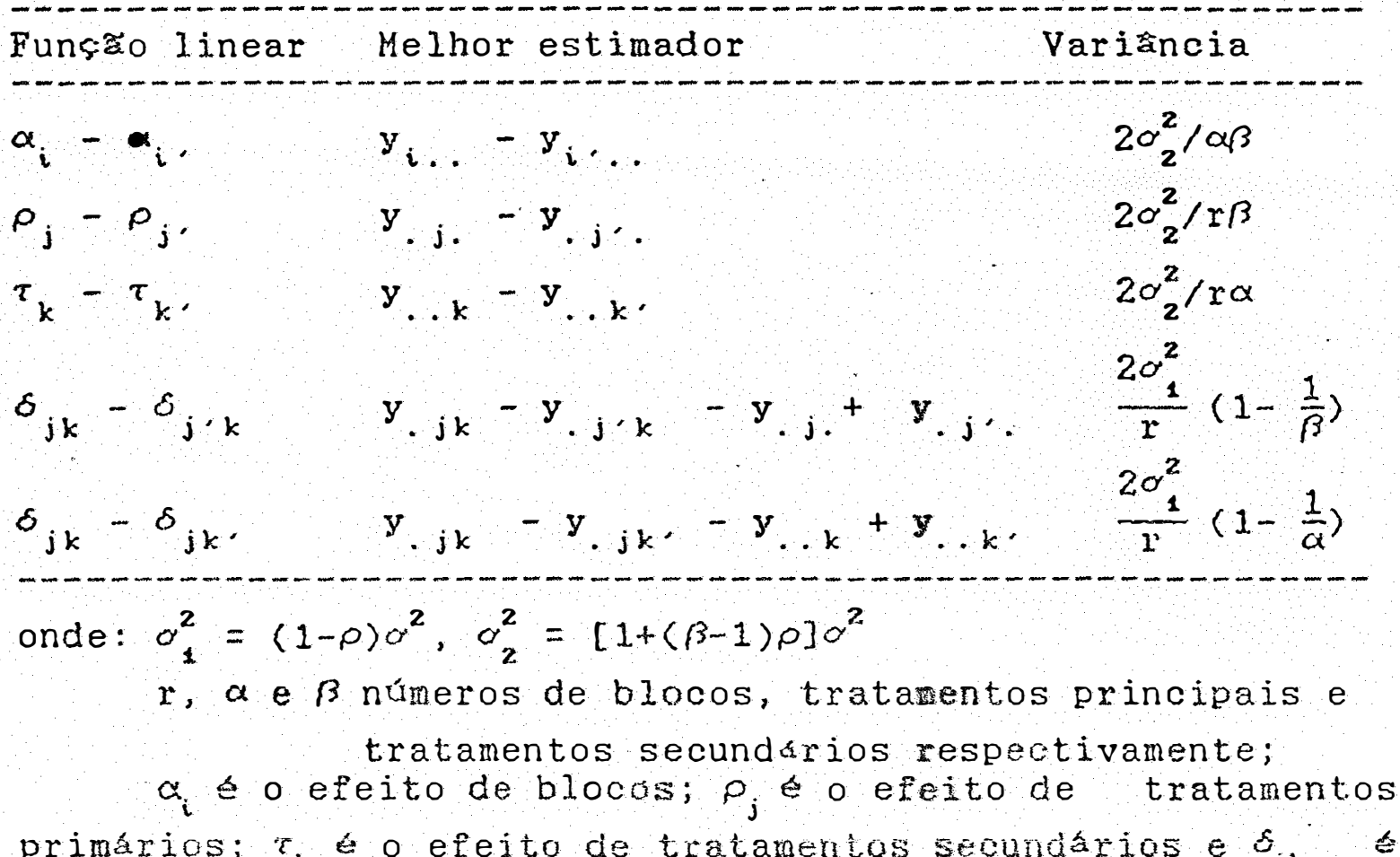
o efeito da interação;

$y_{i}$, média do i-ésimo bloco; $y$, ,. média do j-esimo tratamento primário; $\mathrm{y} \ldots k$ media do k-esimo tratamento secundário e y.jk media da interaça.

Em algumas situaçðes FEDERER (1955), propōs que, se os niveis do pator $A$ szo comparados em um delineamento em blocos casualizados, o residuo(a) (QM interaça repetiça $\times$ A) e o erro apxopriado para compração do efeito $A$, enquanto o residuo(b), que é composto pelas interaços de repetiçăo $\times$ fator $B$ mais repeticão $\times A \times B$, - erro apropriado para testar a interaça $A x A$ e para testar o efeito $B$.

A eficiência dos experimentos em parcelas subdivididas, de acordo com FEDERER (1955), em relação ao delineamento em blocos casualizados sobre as comparaçses B e A $x$ B foi dada por

$$
\frac{(v-1) E_{a}+v(u-1) E_{b}}{(v u-1) E_{b}}=\frac{E_{b}^{\prime}}{E_{b}}
$$

onde $\mathrm{y}=$ número de tratamentos prinnipais: $n=$ número de 
tratamentos na subparcela, $E_{a}=Q M$ erro(a) e $E_{b}=Q M$ erro(b). E, a eficizncia sobre o efeito A ou comparaçes nas parcelas foi dada por $E_{e}^{\prime} / E_{a}$.

\subsection{Experimentos em blocos incompletos parcialmente bal anceados (PBIB)}

\subsubsection{Caracterizactao e definicáo}

Os delineamentos em blocos incompletos parcialmente balanceados ou simplesmente PBIB foram introduzidos por BOSE \& NAIR (1839), como uma generalizacão dos delineamentos em blocos incompletos balanceados (BIB), iniciados por YATES (1936), como uma forma de arranjar um grande número de variedades.

Segundo BOSE \& NAIR (1939) e NAIR (1952), um delineamento em blocos incompletos $e$ dito ser parcialmente balanceado se: i) existem v tratamentos arranjados em b blocos com b unidades experimentais com diferentes tratamentos em cada bloco; ii) cada tratamento ocorre em r blocos; iii) com relação a um tratamento qualquer os demais podem ser divididos em $m$ grupos de tamanhos $n_{2}, n_{2}, \ldots, n_{m}$ de tal forma que os tratamentos do i-ésimo grupo ocorram juntos com um dado tratamento em $\lambda_{i}$ blocos. Os tratamentos do i-esimo grupo são denominados de i-ésimos associados do tratamento em questão, sendo os valores de $n_{1}, n_{2} ; \ldots, n_{m}, \lambda_{1}, \lambda_{2}$, $\ldots, \lambda_{m}$ independentes do tratamento considerado; iv) Se o tratamento $A$ é o i-ésimo associado do tratamento $B$, então o tratamento $B$ e o i-ésimo associado de $A$. Se $A$ e $B$ são os i-ésimos associados, então o número de tratamentos que são comuns aos j-ésimos associados de $A$ e aos k-ésimos associados de $B$ e $\mathrm{p}_{j k}^{i}$, e são independentes do par de tratamentos considerado. Tambem, tem-se que $p_{j k}^{i}=p_{k j}^{i}$.

Os simbolos $v, b, r, k, \lambda_{1}, \lambda_{2}, \ldots, \lambda_{m}, n_{1}$, $n_{2}, \ldots, n_{m}$ são denominados parâmetros do primeiro tipo ou parametros do delineamento, $e$ os numeros $\mathrm{p}_{j k}^{i}(i, j, k=1$, $2, \ldots . m$ m) são denominados de parâmetros do segundo tipo. Assim, existem $2 m+4$ parâmetros do primeiro tipo e $m^{2}(m+1) / 2$ 
parámetros do segundo tipo. Por conveniência, assume-se que qualquer tratamento é zero-ésimo associado dele mesmo e de nenhur outro.

Os autores mencionaram, ainda, que no caso particular, quando $\mathrm{m}=1$, o delineamento se transforma em blocos incompletos balanceados; o mesmo ocorrendo quando $\lambda_{1}$ $=\lambda_{2}=\ldots=\lambda_{m}=\lambda$.

De acordo com BOSE \& NAIR (1939), RAO (1947) e NAIR (1952) as sequintes propriedades se verificam entre os parametros de um PBIB:

i) $\mathrm{vr}=\mathrm{bk}$;

ii) $n_{1}+n_{2}+\ldots+n_{m}=v-1$;

iii) $n_{1} \lambda_{1}+n_{2} \lambda_{2}+\ldots+n_{m} \lambda_{m}=r(k-1)$;

$$
\begin{aligned}
& \text { iv) } \sum_{k=1}^{m} p_{j k}^{i}=\left\{\begin{array}{l}
n_{j} \text { para } i \not k_{j} \\
n_{j}-1 \text { para } i=j
\end{array}\right. \\
& \text { v) } n_{i} p_{j k}^{i}=n_{j} p_{i k}^{j} .
\end{aligned}
$$

o conceito de esquema de associaça representa

uma regra fundamental na análise e classificaça dos delineamentos parcialmente balanceados. Este conceito foi introduzido por BOSE \& SHIMAMOTO (1952), e a definição dos delineamentos em PBIB foi refeita neste contexto.

As definiçðes de um esquema de associação com m-classes de associados e de um delineamento PBIB, segundo NIGAM et alii (1989) são dadas a seguir.

DEFINIÇO: Dados v tratamentos, uma relação é dita ser um esquema de associaça com $m$-classes se satisfaz às seguintes condiçes:

i) Quaisquer dois tratamentos são primeiros, segundos, .... ou m-ésimos associados e a relacão de associação é simetrica. Isto $\varepsilon$, se o tratamento $\alpha \hat{\varepsilon}$ o i-ésimo associado do tratamento $\beta$, então $\beta \in$ tamber o $\dot{i}$-ésimo associado de $\alpha$.

ii) Cada tratamento $\approx$ possue $n_{i}$ i-śsimos assooidos e 0

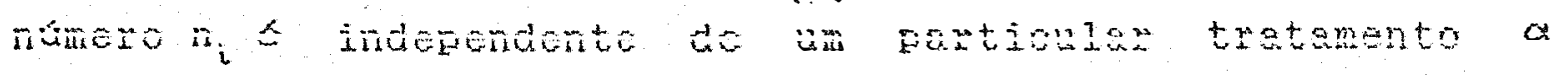


escolhido.

iii) Se quaisquer dois tratamentos a e $\beta$ são os $i$-esimos associados, então o número de tratamentos que são j-ésimos associados de $\approx$ e $k$-śsimos associados de $\beta \Leftarrow p_{j k}^{i}$ e o número $\mathrm{p}_{j k}^{i} \Leftarrow$ independente do par do i-ésimo associado $\alpha$ e $\beta$.

DEFINIÇÃO: Dado um esquema de m-associados, um delineamento PBIB com m-classes de associa̧ão, baseado no esquema de associação, é um arranjo de $\mathbf{v}$ tratamentos em b blocos tais que: i) Cada bloco contêm $k$ distintos tratamentos, com $k$ < Y;

ii) Cada tratamento ocorre em $r$ blocos;

iii) Quaisquer dois tratamentos que são i-ésimos associados ocorrem juntos em $\lambda_{i}$ blocos $(i=1,2, \ldots, m)$, onde $\lambda_{i} e$ uma constante independente do par de tratamentos.

\subsection{PBIB com duas classes de associados - PMIBC2)}

\subsubsection{Caracterizacia}

Do ponto de vista prático, os delineamentos em blocos incompletos parcialmente balanceados com duas classes de associados, ou simplesmente PBIB(2), são os mais importantes e os mais comumente utilizados e por isso säo de especial interesse; uma lista desses delineamentos foi preparada por BOSE et alii (1954) e foi posteriormente revista por CLATHORTHY (1967) e DEY (1988), entre outros. Nesse caso, quando $m=2$, existem oito parame- tros do primeiro tipo $\left(v, b, r, k, \lambda_{1}, \lambda_{2}, n_{1}, n_{2}\right)$, e seis do segundo tipo $\left(p_{j k}^{i} ; i, j, k=1,2\right)$. Os parametros do segundo tipo podem ser apresentados cono elementos de duas matrizes simetricas, tais como

$$
P_{1}=\left[\begin{array}{cc}
p_{11}^{1} & p_{12}^{1} \\
p_{21}^{1} & p_{22}^{1}
\end{array}\right] \text { e } P_{2}=\left[\begin{array}{cc}
p_{11}^{2} & p_{12}^{2} \\
p_{21}^{2} & p_{22}^{2}
\end{array}\right]
$$

A relação de associaçăo entre as tratamentos, referida na deliniça de PBIB, pode ser estabelecida atraves de una regra, que informa que tipo de associados são dois dados tratamentos. Quando $\mathrm{m}=2$, segundo Buse \& sHimimuto 
(1952) a regra especifica se dois tratamtos são primeiros ou segundos associados. Como ilustracão os autores consideraram $v=10$ tratamentos, $m=2$, e os tratamentos $a, b$, $c, d, e, f, g, h, i, j$ estão arranjados no seguinte esquema:

$\begin{array}{lllll}* & \mathrm{a} & \mathrm{b} & \mathrm{c} & \mathrm{d} \\ \mathrm{a} & * & \mathrm{e} & \mathrm{f} & \mathrm{g} \\ \mathrm{b} & \mathrm{e} & * & \mathrm{~h} & \mathrm{i} \\ \mathrm{c} & \mathrm{f} & \mathrm{h} & * & \mathrm{j} \\ \mathrm{d} & \mathrm{g} & \mathrm{i} & \mathrm{j} & *\end{array}$

Supondo que a regra de associaça s: dois tratamentos s̃o primeiros associados somente se eles ocorrem juntos na mesma coluna do esquema, em caso contrário são segundos associados. Pode-se verificar que cada tratamento possui 6 primeiros associados $\left(n_{1}=6\right)$ e 3 segundos associados $\left(n_{2}=3\right)$. Para os tratamentos a, $c, 1$ e h, tem-se:

Tratamento Primeiros associados Segundos associados
a
$b, c, d, e, f, g$
$i, j, h$
c
$a, b, d, f, h, j$
$e, g, i$
i
$b, e, h, d, g, j$
$a, c, f$
h
$b, e, i, c, e, j$
$a, d, b$

Os tratamentos a e c são primeiros associados.

o número de tratamentos que são primeiros associados de a e também primeiros associados de $c$ é 3 (b, d,f), portanto, $\mathrm{p}_{11}^{1}=3$. Verifica-se que o número de tratamentos que $s \widetilde{a}$ segundos associados de a e c é 1 (i), portanto $p_{22}^{1}=1$. Também, e e g são os únicos tratamentos comuns aos primeiros associados de a e segundos associados de $c$, logo $\mathrm{p}_{12}^{1}=2$. Desse modo, facilmente, verifica-se a condiçăo de constância de $\mathrm{p}_{11}^{1}, \mathrm{p}_{12}^{1}$ e $\mathrm{p}_{22}^{1}$, e a simetria de $\mathrm{p}_{12}^{1}=\mathrm{p}_{21}^{1}$, para qualquer par de tratamentos.

Do mesmo modo, tomando-se dois tratamentos segundos associados, vexifica-se que $\mathrm{p}_{11}^{2}=4, \mathrm{p}_{12}^{2}=\mathrm{p}_{21}^{2}=2$ e $\mathrm{p}_{22}^{2}=0$. 


\subsubsection{Classificacáo}

Os delineamentos PBIB(2) com duas classes de associados foram classificados por BOSE \& SHIMAMOTO (1952) de acordo com a natureza do esquema de associaça entre os tratamentos, em cinco tipos: grupo divisivel, triangular, quadrado latino, clclico e simples. Alem deles NIGAM et alii (1989) classificaram em quatro tipos: grupo divisivel, triangular, quadrado latino e geometria parcial e consideraram os três primeiros como os mais importantes.

\subsubsection{Tipo grupo divisivel}

Um PBIB com duas classes de associados e do tipo grupo divisivel (GD) se $v=m n$ tratamentos satisfazem o seguinte esquema de associasão: os $v$ tratamentos são divididos em m grupos de tamanho $n$, tal que, quaisquer dois tratamentos do mesmo grupo sao os primeiros associados e os de grupos diferentes são segundos associados. Os parâmetros do esquema sao $n_{1}=n-1, n_{2}=n(m-1)$ e

$$
P_{1}=\left\{p_{j k}^{1}\right\}=\left[\begin{array}{cc}
n-2 & 0 \\
0 & n(m-1)
\end{array}\right] \quad P_{2}=\left\{p_{j k}^{2}\right\}=\left[\begin{array}{ll}
0 & n-1 \\
n-1 & n(m-2)
\end{array}\right]
$$

Os autovalores de $N^{\prime}$ são $\theta_{0}=k r, \theta_{1}=r-\lambda_{1}$ e $\theta_{2}=\mathrm{kr}-\mathrm{v \lambda _{2 }}$ com multipliaidade $\rho_{0}=1, \rho_{1}=m(n-1)$ e $\rho_{2}=m-1$ respectivamente.

De acordo com os autovalores $\theta^{\prime} \mathrm{s}$, BOSE \& CONNOR (1952) classificaram os delineamentos do tipo GD em tros classes:

i) singular $(S)$, se $\theta_{1}=0$ ou $r=\lambda_{1}$

ii) semiregular (SR), se $\theta_{1}>0, \theta_{2}=0$ ou $r>\lambda_{1}$ e $r k=v \lambda_{2}$ iii) regular (R), se $\theta_{1}, \theta_{2}>0$ ou $r>\lambda_{1}$ e rk> $>\lambda_{2}$

\subsubsection{Tipo triangular}

Um PBIB com duas classes de associados é do tipo triangular se os $\mathrm{v}=\mathrm{n}(\mathrm{n}-1) / 2$ tratamentos satisfazem 0 seguinte esquema de associaça: os $\mathrm{v}$ tratamentos são axranjados om uma matria do n linhas por n colunas, tal gue, 
a diagonal principal é deixada em branco e as $n(n-1) / 2$ posiços do triángulo acima da diagonal são ocupadas pelos $n(n-1) / 2$ tratamentos. Do mesmo modo, as $n(n-1) / 2$ posiçбes do triangulo abaixo da diagonal são ocupadas, de modo que a matriz seja simetrica. Os tratamentos que ocorrem na mesma linha e mesma coluna são primeiros associados, e caso contrário, são segundos associados.

Os parametros do esquema sao $n_{1}=2(n-2)$, $\mathrm{n}_{2}=(\mathrm{n}-2)(\mathrm{n}-3) / 2$,

$P_{1}=\left[\begin{array}{ll}n-2 & n-3 \\ n-3 & (n-3)(n-4) / 2\end{array}\right]$ e $P_{2}=\left[\begin{array}{ll}4 & 2 n-8 \\ 2 n-8 & (n-4)(n-5) / 2\end{array}\right]$

onde $n \geq 5$; quando $n=4, \mathrm{p}_{12}^{2}=0$, e o delineamento $\leqslant$, tamb $\hat{s}_{m}$, do tipo GD.

Os autovalores da matriz de incidência $\mathrm{NN}^{\circ}$ alsm do $\theta_{0}=k r$, seo $\theta_{1}=r+(n-4) \lambda_{1}-(n-3) \lambda_{2} e \theta_{2}=\left(x-2 \lambda_{2}+\lambda_{2}\right)$, com multiplicidade $\hat{\rho}_{1}=(n-1)$ e $\hat{\rho}_{2}=n(n-3) / 2$ respectivamente.

Por exemplo, quando $n=4$, os seis tratamentos podem ser arranjados como:

$\begin{array}{llll}* & 1 & 2 & 3 \\ 1 & * & 4 & 5 \\ 2 & 4 & * & 6 \\ 3 & 5 & 6 & *\end{array}$

Os primeiros associados de 1 s a os tratamentos: $2,3,4,5$ e o segundo associado e o tratamento: 6 .

\subsubsection{Tipo quadrado latino}

Um PBIB com duas classes de associados é do tipo quadrado latino com $i$ restriçres $\left(L_{i}\right)$, se os $v=n^{2}$ tratamentos satisfazem ao seguinte esquema de associacão: os $\mathrm{v}=\mathrm{n}^{2}$ tratamentos são arranjados em uma matriz quadrada, de ordem $n$, e i-2 quadrados latinos mutuamente ortogonais, de dimensöes $n \times n$, são superpostos na matriz quadrada para identificar os primeiros e segundos associados de qualquer tratamento. Dois tratamentos são primeiros associados se e

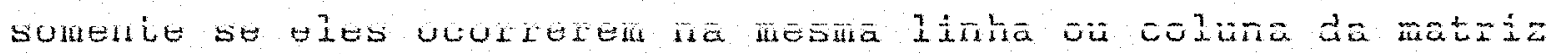


ou corresponderem a mesma letra em qualquer dos $i-2$ quadrados latinos superpostos. Nos demais casos, eles são segundos associados. Para o esquema $L_{2}$, dois tratamentos $s \widetilde{a}_{0}$ primeiros associados se eles ocorrerem na mesma linha ou coluna. Para esse esquema tem-se

$$
\begin{aligned}
v=n^{2}, n_{1}=i(n-1), & n_{2}=(n-i+1)(n-1), \\
P_{1} & =\left[\begin{array}{ll}
i^{2}-3 i+n & (i-1)(n-i+1) \\
(i-1)(n-i+1) & (n-i)(n-i+1)
\end{array}\right] \\
P_{2} & =\left[\begin{array}{ll}
i(i-1) & i(n-i) \\
i(n-i) & (n-i)^{2}+(i-2)
\end{array}\right]
\end{aligned}
$$

Os autovalores da matriz de incidencia NN. alem de $\theta_{0}=k r$ s ̃o $\theta_{1}=r+(n-i) \lambda_{1}-(n-i+1) \lambda_{2}$ e $\theta_{2}=r-i \lambda_{1}+(i-1) \lambda_{2}$ com multiplicida $\rho_{1}=i(n-1)$ e $\quad p_{2}=(n-i+1)(n-1)$ respectivamente.

- esquema $L_{z}$, é o caso once os tratamentos estao arranjados en una sequencia quadrada. Por exemplo, se $n=3$, tem-se

$$
\left[\begin{array}{lll}
1 & 2 & 3 \\
4 & 5 & 6 \\
7 & 8 & 9
\end{array}\right]
$$

\subsubsection{Geometria parcial $(r, k, t)$}

Uma geométria parcial ( $r, k, t$ ) é definida como um sistema de $r$ pontos e b linhas com suas relaçós de incidercia satisfazendo os seguintes axiomas:

Axioma 1. Qualquer dois pontos distintos são incidentes con nzo mais que uma linha.

Axioma 2. Cada ponto $e$ incidente com $r$ linhas.

Axioma 3 . Cada linha 6 incidente com $k$ pontos.

Axioma 4. Se o ponto $P$ não e incidente em $p$, entao existem exatamente $t$ linhas através de $P$ que interceptam $p$.

Identificando $\mathbf{v}$ pontos como $\mathbf{v}$ tratamentos $\mathrm{e} b$ linhas como b blocos, pode-se veripicar que qualquer geométria parcial corresponde a um PBIB do tipo simples com duas ciasses de associados e com os paranetros: 
$v=k[(r-1)(k-1)+t] / t, \quad b=r[(r-1)(k-1)+t] / t, r, k, \lambda_{1}=1, \lambda_{2}=0$, $n_{1}=r(k-1), \quad n_{2}=(r-1)(k-1)(k-t) / t, \quad p_{11}^{1}=(t-1)(r-1)+k-2$, $p_{12}^{1}=(r-1)(k-1), p_{11}^{2}=r t, p_{12}^{2}=r(k-t-1), 1 \leq t \leq m i n(r, k)$.

Para o esquema de associação acima, quaisquer dois pontos sao primeiros associados quando. sâo incidentes com uma linha da geometria, e são segundos associados guando não são incidentes com a linha.

Os autovalores da matriz de incidência $N^{\prime}$ são $\theta_{0}=r k, \quad e_{1}=r+k-t-1$ e $\theta_{2}=0$ com multiplicidades $\rho_{0}=1$, $P_{1}=[r k(r-1)(h-1)] / t(k+r-t-1)$ e $P_{2}=v-1-P_{1}$, respectivamente. Como as multiplicidades dos autovalores necessitam ser números inteiros, segue-se que uma condiça necessária para a existencia de uma geometria parcial $(r, k, t)$ e que $r k(r-1)(k-1) / t(k+r-t-1)$ seja um inteiro positivo.

A definiçăo de cala tipo bem como seus respectivos esquemas pode ser vista com mais detalhes em BARBOSA (1986), BOSE \& SHIMAMOTO (1952) e RIBOLDI (1988), entre outros.

\subsection{Experimentos em reticulados quadrados}

\subsubsection{Caracterizaça}

o delineamento reticulado quadrado permite dispor $v=k^{2}$ tratamentos em blocos de $k$ unidades or bloco. Das, que o número de tratamentos tem que ser um quadrado perfeito e, que os tratamentos de um bloco em uma repetiça se distribuen em diferentes blocos nas outras repetiçఠes, constituindo as chamadas repetiços ortogonais ou arranjos básicos. Assim, se $k$ for um número primo ou potencia de primo, têm-se $k+1$ repetiç̋es ortogonais, e o conjunto das $k+1$ repetiços constitui o reticulado quadrado balanceado.

$$
\text { Um reticulado quadrado simples "simple }
$$

lattice" é um PBIB com duas classes de associados baseado no esquema de associaça $I_{2} ;$ do mesmo modo o reticulado quadrado triplo é baseado no esquema $I_{b}$. Esses esquemas

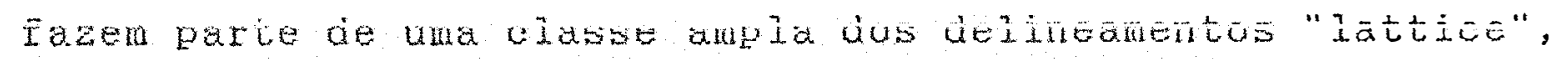


introduzida por YATES (1936), que foram também chamados de delineamentos "quasifatorial".

\subsubsection{Classificacao}

Os reticulados quadrados podem ser classificados quanto a repetiç̃o dos axranjos básicos, de acordo com COCHRAN \& COX (1957), em:

i) simples ou duplo: quando são utilizados somente duas repetiçes do reticulado balanceado;

ii) triplo ou trlplice: quando são utilizados somente tres repetiçres;

iii) quádruplo: quando são utilizados somente quatro repetiçres do esquema balanceado ou quando é utilizado o reticulado simples duplicado;

iv) quintuplo: quando sº utilizados cinco repetictes;

v) para um maior número de repetiços, os autores sugerem:

- seis repetiçes: usar o reticulado triplice duplicado ou o reticulado simples triplicado;

- oito repetiçes: usar o reticulado quadruplo duplioado ou o reticulado simples quatro vezes;

- nove repetiçes: usar o reticulado tríplice triplicado;

- dez repetiçoes: usar o reticulado quintuplo duplicado ou o reticulado simples cinco vezes.

RAGHAVARAO (1971), considerou que os delineamentos reticulados quadrados parcialmente balanceados, pertencem ao esquema de associaşa do tipo quadrado latino com i restriçes $\left(L_{i}\right)$, com os parametros $n_{1}=i(n-1), n_{2}=$ $(n-i+1)(n-1)$ e $p_{11}^{i}=i^{2}-3 i+n$. Considerou ainda, que quando os $v=n^{2}$ tratamentos podem ser repetidos $s$ vezes, obte $\epsilon_{m}-s e$ um delineamento com os parametros $v=n^{2}, b=s n i, r=s i, k=n, \quad \lambda_{1}=s$ e $\lambda_{2}=0$.

\subsection{Analise da varigracia intrablocos}

A análise intrablocos, em que somente as comparaçes entre parcelas do mesmo bloco são usadas nas estimativas dos efeitos de tratamentos, para os delineamentos PBIB pode ser vist em CHAKRABARII (1862), RAGHAVARAO 
(1971), PIMENTEL GOMES (1985), DAS \& GIRI (1986) \& IEMMA (1987), dentre outros.

PIMENTEL GOMES (1985), salientou que a análise intrablocos pode ser utilizada para qualquer tipo de experimento em blocos incompletos e se baseia en metodos exatos.

Sabe-se que o sistema de equaças normais, eliminando-se o efeito de blocos, é dado por

$$
\mathrm{C} \hat{\tau}=Q \text {, }
$$

onde: $C=R-N K^{-1} N^{\prime}=r I_{(V)}-N N^{\prime} / k$,

$$
\mathrm{NN}=\left[\begin{array}{cccc}
r & \lambda_{12} & \cdots & \lambda_{1 v} \\
\lambda_{12} & r & \cdots & \lambda_{2 v} \\
\cdots & \cdots & \cdots & \cdots \\
\lambda_{1 v} & \lambda_{2 v} & \cdots & \mathrm{r}
\end{array}\right]
$$

Assim, $C$ e uma matriz singulax de caractexistica (ou posto incompleto) $v-1$, constituida pelos seguintes elementos:

$$
c_{i i^{\prime}}= \begin{cases}r(k-1) / k & \text { se } i=i^{\prime} \\ -\lambda_{i, i} / k & \text { se } i \neq j^{\prime}\end{cases}
$$

sendo $\lambda_{i i}$ o número de vezes que os tratamentos $i e i$. ocorrem juntos num mesmo bloco.

$E \circ Q \quad 0$ vetor dos totais ajustalos de tratamentos, constituido pelos seguintes elementos

$$
Q_{i}=T_{i}-A_{i} / k
$$

onde $T_{i}$ e a total das observaçes do tratamento $i$ e $A_{i} \Leftrightarrow$ a soma dos totais das observaçes dos blocos que contêm o tratamento i.

$\mathrm{Na}$ obtença de estimadares dos efeitos ajustados de tratamentos de um PBIB, uma dificuldade que ocorre, está em apresentar regras gerais que facilitem a analise. Para contornar esse problema, una das altexnativas é o uso de restriçoes não estimáveis na solução, de modo que se complete o post• da matriz C.

ROSF \& NATR (1939): impondo a restricã não 
estimável $\sum_{i=1}^{v} \hat{t}_{i}=0$ para os $\operatorname{PBIB}(2)$, apresentaram a seguinte expressão para $\hat{t}_{i}$ :

$$
\hat{t}_{i}=k\left[B_{2 z} Q_{i}-B_{12} S_{1}\left(Q_{i}\right)\right] / \Delta_{1}, i=1,2, \ldots, v,
$$

onde:

$$
\begin{aligned}
B_{22} & =r(k-1)+\lambda_{2}+\left(\lambda_{2}-\lambda_{1}\right)\left(p_{11}^{1}-p_{11}^{2}\right) ; B_{12}=\lambda_{2}-\lambda_{1} ; \\
\Delta_{1} & =A_{12} B_{22}-A_{22} B_{12} ; \\
A_{12} & =r(k-1)+\lambda_{2} ; A_{22}=\left(\lambda_{2}-\lambda_{1}\right) p_{12}^{2} ;
\end{aligned}
$$

$S_{1}\left(Q_{i}\right) \Leftarrow a$ soma dos $Q^{\prime}$ s correspondentes aos $n_{1}$ tratamentos que são primeiros associados do tratamento $i$.

Mas, se $n_{1} \geq n_{z}$, uma expressão mais conveniente $\Leftrightarrow$ dada por

$$
\hat{t}_{i}=k\left[B_{21} Q_{i}-B_{11} S_{2}\left(Q_{i}\right)\right] / \Delta_{2}
$$

onde: $B_{21}=r(k-1)+\lambda_{1}+\left(\lambda_{1}-\lambda_{2}\right)\left(p_{22}^{2}-p_{22}^{1}\right) ; B_{11}=\lambda_{1}-\lambda_{2}$;

$$
\begin{aligned}
& \Delta_{2}=A_{11} B_{21}-A_{21} B_{11} ; \\
& A_{11}=r(k-1)+\lambda_{1} ; A_{21}=\left(\lambda_{1}-\lambda_{2}\right) p_{12}^{1} ;
\end{aligned}
$$

$S_{2}\left(Q_{i}\right)$ é a soma dos $Q$ 's relativos aos $n_{2}$ tratamentos que são segundos associados do i-ésimo tratamento.

Expressoes semelhantes as de BOSE \& NAIR (1939) forain apresentadas por RA (1947), com a diferenca de que define $S_{j}\left(Q_{i}\right)(i=1, \ldots, v ; j=1, \ldots, m)$ como a soma dos $Q^{\prime} s$ para o i-ésimo tratamento e seus $j$-esimos associados. Assim, as expressð̃es de $\hat{v}_{i}$ são dadas por:

$$
\hat{v}_{i}=k\left[\left(B_{22}+B_{12}\right) Q_{i}-B_{12} S_{1}\left(Q_{i}\right)\right] / \Delta_{1}
$$

quando $n_{1} \leq n_{2}$, e

$$
\hat{v}_{i}=k\left[\left(B_{21}+B_{11}\right) Q_{i}-B_{11} S_{2}\left(Q_{i}\right)\right] / \Delta_{2}
$$

quando $n_{1} \geq n_{2}$.

Para os experimentos em reticulados quadrados PIMENTEL GOMES ( 1354 ), apresentou a seguinte expressão para obter o efeito de $\hat{t}_{i}$

$$
\hat{t}_{i}=\frac{1}{m} Q_{i}+\frac{1}{m(m-1) k}\left[S_{1}\left(Q_{i}\right)+S_{2}\left(Q_{i}\right)+\ldots+S_{m}\left(Q_{i}\right)\right]
$$


onde: $S_{j}\left(Q_{i}\right)$ e a soma dos $Q^{\prime} s$ de todos os $k$ tratamentos que ocorrem no mesmo bloco da $j$-ésima repetiça $(j=1, \ldots, m)$ em que aparece o i-ésimo tratamento.

Para o caso geral, com m classes de associados, CHAKRABARTI (1982) apresentou para o i-ésimo tratamento a seguinte expressão:

$$
r(k-1) \hat{t}_{i}=k Q_{i}+\sum_{j=1}^{m} S_{j}\left(Q_{B}\right) \sum_{i=1}^{m} \lambda_{i} f_{i j}
$$

ande: $S_{j}\left(Q_{\theta}\right) \in$ a soma dos $Q^{\prime} s$ correspondentes aos $n_{j}$ tratamentos que são j-ésimos associados do s-ésimo tratamento; e $P_{i j}$ que satisfaz a expressæo: $S_{i}\left(\hat{\tau}_{\theta}\right)=f_{i 1} S_{1}\left(Q_{B}\right)+f_{i 2} S_{2}\left(Q_{g}\right)+\ldots+f_{i m} S_{m}\left(Q_{g}\right)$.

De acordo com JOHN (1980), uma soluça do SENR $C \hat{\tau}=Q$ e $\hat{\tau}=\Omega Q$, onde $\Omega$ e uma matriz inversa generalizada simetrica de $C$.

E, ainda, RIBOLDI (1988) considerou gue uma matriz inversa de Moore-Penrose $(\Omega)$ possui os seguintes elementos:

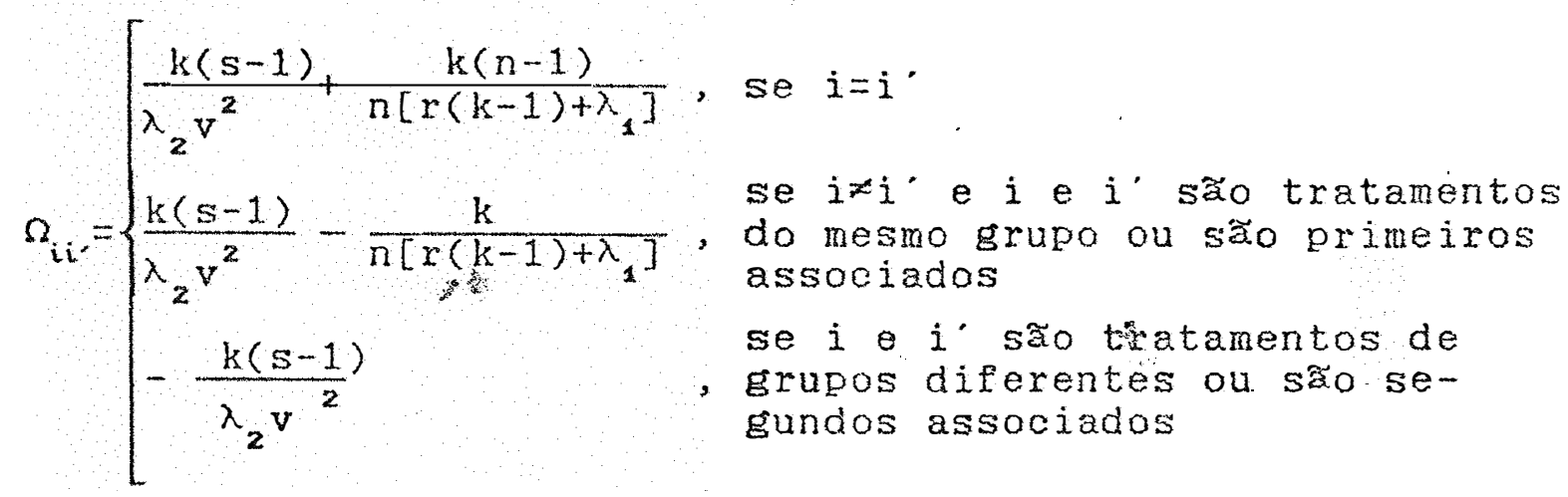

A soma de quadrados de tratamentos ajustada para blocos, foi obtida por $\operatorname{SQT}(a j)=.\hat{\tau} \cdot Q=\sum_{i=1}^{V} \hat{\tau}_{i} Q_{i}$ de acordo com PIMENTEL GOMES (1954) e CHAKRABARTI (1962), entre outros.

Detalhes de sua utilizaça na prática, bem como vários aspectos teóricos, como deducão de expressós para o efeito de tratamentos săo dados em JoHN (1980),

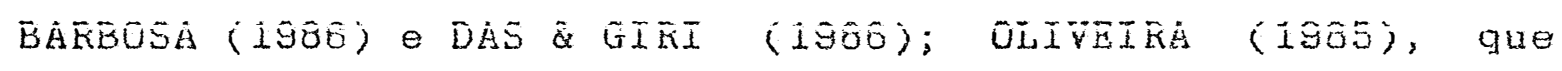


deduziu expressరes para a estimativa dos efeitos de tratamentos, no caso de PBIB com tratamentos comuns em todos os blocos e, RIBOLDI (1988), que apresentou a decomposiça da soma de quadrados de tratamentos ajustada em $v-1$ contrastes ortogonais.

\subsection{Estimabilidade}

Na experimentação agronōmica, é de grande importancia a obtença das médias dos tratamentos; nos experimentos em PBIB, um problema que ocorre, está em verificar se os contrastes entre médias ajustadas são estimáveis. Para isso, sao necessários alguns conceitos sobre delineamentos conexos.

Um delineamento em blocos é definido como conexo por RAGHAVARAO (1971), se dados dois quaisquer tratamentos, por exemplo 1 e $i '$, é possivel construir uma cadeia de tratamentos $i=i_{0}, i_{1}, \ldots, i_{n}=i$, tais que, todos os pares consecutivos de tratamentos ocorram juntos em um bloco. Uma regra prática foi apresentada por SEARLE (1971): i) forma-se uma tabela de dupla entrada, representando os blocos e os tratamentos; $i i)$ nos pontos correspondentes à interseça do bloco $j$ com o tratamento $t$, coloca-se um $X$ se o tratamento $i$ ocorre no bloco $j$; iii) partindo-se de qualquer $X$ e em qualquer sentido unem-se os X's por linhas hor $i-$ zontais e verticais; iv) procedendo-se assim, se todos os $X ' s$ forem unidos sem interrupção, o delineamento e conexo, caso contrário, não será conexo.

Ainda, segundo CHAKRABARTI (1962), RAGHAVARAO (1971) e NIGAM et alii (1989), um delineamento em blocos com $v$ tratamentos será conexo somente se o posto da matriz C do sistema de equaçoses normais reduzidas (SENR) possuir caracteristica $x[C]=v-1$; e OGAWA (1974), demonstrou que, se o delineamento é conexo, então todos os contrastes entre médias ajustadas de tratamentos são estimáveis.

KAGEYAMA (1982), apresentou a seguinte regra 
prática para verificar se um $\operatorname{PBIB}(2)$ é conexo: i) se $\lambda_{1}>\lambda_{2}$ então $\lambda_{2}$ e $p_{11}^{2}$ não devem ser nulos ao mesmo tempo; ii) se $\lambda_{1}<\lambda_{2}$, entao $\lambda_{1}$ e $p_{22}^{1}$ náo devem ser nulos ao mesmo tempo. De acordo com essas condiçes, o autor determinou que: i) os delineamentos do tipo triangular, quadrado latino e clclicos säo sempre conexos; ii) os delineamentos do tipo grupo divisivel com $\lambda_{1}<\lambda_{2}$ s $\widetilde{Z}_{0}$ sempre conexos, e quando $\lambda_{1}>\lambda_{2}$ ser $\tilde{a}_{0}$ conexos somente se $\lambda_{2}$ näo for nulo.

A variancia de um contraste elementar de tratamentos, nos delineamentos com duas classes de associados, conforme BOSE \& NAIR (1939), RAO (1947) e NAIR (1952), e dada por:

$v\left[\hat{t}_{i}-\hat{t}_{i}\right]=\left\{\begin{array}{l}2 \mathrm{kB}_{21} \sigma^{2} / \Delta_{1}, \text { se i e i s so primeiros associados } \\ 2 \mathrm{kB} \mathrm{a}_{2} \sigma^{2} / \Delta_{2}, \text { se } i \text { e i s sa sesundos associados }\end{array}\right.$ Sendo, $B_{22}=B_{21}+\lambda_{1}-\lambda_{2}$ e $\lambda_{1}>\lambda_{2}$ entao os tratamentos primeiros associados tem uma variancia menor do que os segundos assooiados, pois $\Delta_{1}=\Delta_{2}$.

Na solusão de BOSE \& SHIMAMOTO (1952), tem-se $v\left[\hat{t}_{i}-\hat{t}_{i}\right]=\left\{\begin{array}{l}2\left(k-c_{1}\right) \alpha^{2} / x(k-1), \text { se } i \text { e i são } 1^{\text {os }} \text { associados } \\ 2\left(k-c_{2}\right) \alpha^{2} / r(k-1), \text { se } i \text { e i' são } 2^{\text {os associados }}\end{array}\right.$ E ainda, BARBOSA (1386), apresentou as seguintes expressoes:

(i) $s e n_{1}<n_{2}$

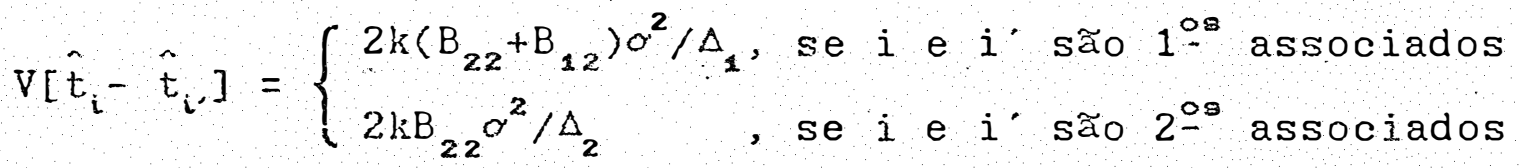
(ii) se $n_{1}>n_{2}$

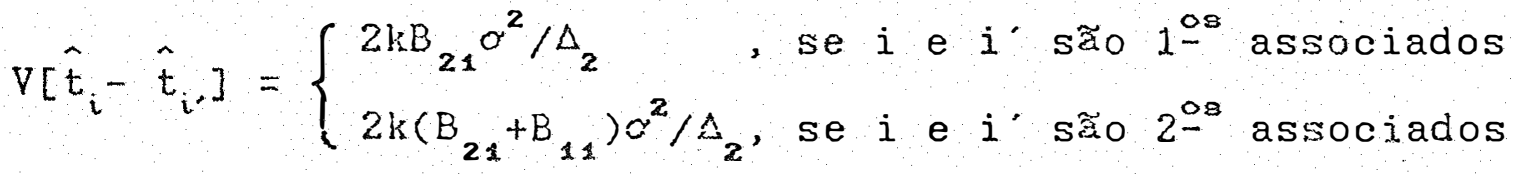
Como cada um dos v tratamentos têm $n_{i}$ i-ésimos associados, entzo tem-se vn pares de tratamentos. Mas, cada par e contado duas vezes, logo exiten $\mathrm{vn}_{\mathrm{l}} / 2$ comparařzes entre os tratamentos primeiros associados e $\mathrm{vn}_{2} / 2$ 
comparaçరes entre os segundos associados. Uma variancia media de todas comparaçరes entre contrastes elementares, de acordo com BOSE \& NAIR (1939) é dada por:

$$
V=2 k c^{2}\left[(v-1) B_{21}+n_{2} B_{11}\right] /\left[(v-1) \Delta_{2}\right]
$$

Para o caso geral dos delineamentos com m classes de associados, CHAKRABARTI (1962) considerou que se

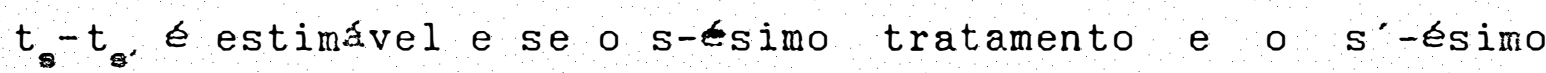
tratamento são i-ésimos associados, então o melhor estimador

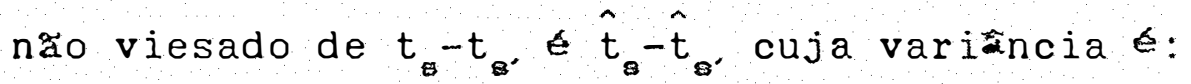

$$
V\left(\hat{t}_{-}-\hat{t}\right)=2 \sigma^{2}\left[k-\sum_{l=1}^{m} \lambda_{l} f_{l i}\right] / r(k-1)
$$

Verifica-se facilmente, que os contrastes elementares na sao estimados com a mesina variancia.

\subsection{Experimentos de parcelas subdivididas em blocos} incompletos

Conforme IEMMA (1981), existe uma certa lacuna quanto as publicaçชes sobre os experimentos em parcelas subdivididas conduzidos em blocos incompletos.

De acordo com GILL (1978), experimentos nos quais animais ou individuos são distribuidos aleatoriamente e então são realizadas avaliaçठes em vários tempos ou espaços nos níveis de um fator ou combinaçoses de dois ou mais fatores, tem-se tornado muito comuns. Conforme o autor, cada animal poderia ser tomado como um bloco incompleto, pois nesse tipo de experimento, um único animal receberia alguns dos tratamentos.

Em algumas zreas de pesquisa, como fisiologia reprodutiva, o autor considerou que as causas de variaça são muitas vezess simplificadas na análise dos dados. Essas simplificaç̋es, não justificadas, são: i) análise dos dados considerando a estrutura fatorial, em vez de uma análise em parcelas subdivididas; ii) análise não considerando a correlação entre os erros, induzida pela repetição das

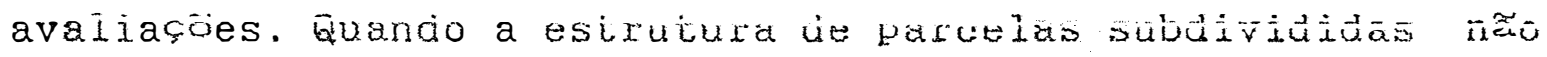


24.

é reconhecida, uma aparente significancia entre médias de tratamentos, pode ser grosseiramente distorcida, e como consequencia, a sensibilidade dos testes pode ser seriamente reduzida.

Quando a correlação entre erros e ignorada, GILL (1878) considerou ue as inferências podem ou não ser distorcidas, dependendo da heterogeneidade das variancias e covariancias dos dados. En muitos experimentos uma suposição de uniformidade é razoável, como o caso onde os animais são tratados igualmente, em relação ao fator $A$. Se os individuos, provavelmente no mostram resposta uniforne, eles podem ser arranjados nos níveis do fator $A$ dentro de cada bloco. Os blocos incompletos são então, grupos de individuos homogêneos; todos os membros de un bloco possuem o mesmo nivel de um pator.

ROBINSON (1387), considerou um experimento envolvendo $t$ niveis do tratamento principal e $s$ niveis do tratamento secundário, sendo o número de subparcelas em cada parcela uma restriçao, ou seja, $k$ 's s. Assim, cada nivel do fator principal foi aplicado em $r$ parcelas e os $s$ niveis do tratamento secundário foram arranjados segundo um esquema de blocos incompletos balanceados, considerando cada parcela como um bloco incompleto. Em um particular nivel do fator $A$, cada nivel do fator $B$ ocorre $R$ vezes e cada par de niveis do fator $B$ ocorre na mesma parcela $\lambda$ vezes, onde $R=r k / s$ e $\lambda=R(k-1) /(s-1)$.

Usando a notação de BOSE \& NAIR (1939) e RAO (1947), verificou-se que o delineamento proposto acima, é um caso especial de um PBIB com os seguintes parâmetros: $\lambda_{1}=\lambda$, $\lambda_{2}=0, n_{1}=s-1, n_{2}=s(t-1) \quad e$

$$
p_{i j}^{1}=\left[\begin{array}{cc}
s-2 & 0 \\
0 & s(t-1)
\end{array}\right], \quad p_{i j}^{2}=\left[\begin{array}{ll}
0 & s-1 \\
s-1 & s(t-2)
\end{array}\right] \text {. }
$$

Para exemplificar um experimento desse tipo, o autor considerou um ensaio fatorial $3 \times 3$ com duas subparcelas ell cacia parcela. 0 esquella hãu casualizacio füi. 
25.

\begin{tabular}{|c|c|c|}
\hline$a_{0} b_{0} \quad a_{a} b_{1}$ & $a_{1} b_{a} \quad a_{1} b_{1}$ & $a_{2} b_{0} \quad a_{2} b_{1}$ \\
\hline$a_{0} b_{a} \quad a_{0} b_{2}$ & $a_{1} b_{0} \quad a_{1} b_{2}$ & $a_{2} b_{0} \quad a_{2} b_{1}$ \\
\hline$a_{0} b_{1} \quad a_{0} b_{2}$ & $a_{1} b_{1} \quad a_{1} b_{2}$ & $a_{2} b_{1} \quad a_{2} b_{2}$ \\
\hline
\end{tabular}

Com relação a experimentos nos quais os animais ou os individuos $\mathbf{s}$ a fixados aleatoriamente nos niveis de um fator, GILL (1978) considerou o caso onde os animais são tomados como blocos incompletos, e apresentou. o seguinte modelo matemático:

$$
y_{i j k}=\mu+\alpha_{i}+D_{\langle i\rangle j}+\beta_{k}+(\alpha \beta)_{i k}+(D \beta)_{(i) j k}+E_{i j k}
$$

para $i=1,2, \ldots, a ; j=1,2, \ldots, r$ (por $i) ; k=1,2, \ldots, b$ e $n=a r b$;

onde: $y_{i j k} \quad \hat{o}$ o valor observado do $i$-êsimo nivel do tratamento $A \cdot n \cdot j$-ésimo animal (bloco) que recebeu o $k$-ssimo nivol do tratamento $B$;

$\approx_{i} \quad t o$ efeito do i-ésimo nivel do tratamento A ao qual os animais sao designados ao acaso;

$D_{(i) j} e$ o efeito aleatorio do j-ésimo animal dentro do i-esimo nivel do tratamento $A$, e considerado como o erro (a);

$\beta_{k} \Leftrightarrow$ o efeito do k-esimo tratamento $B$ nos animais;

$(\alpha \beta)_{i k} \quad e$ o efeito da interaço entre o i-ésimo nivel do tratamento $A$ e o k-esimo nivel do tratamento $B$, sobre os animais;

$(D R)_{(i) j k} \Leftrightarrow 0$ efeito da interaça entre animais e niveis do tratamento $B$, que deve ser isolado do erro (b);

$E_{i j k} \quad \Leftrightarrow$ érro aleatorio atriburdo a observaçao $y_{i j k}$, correspondente ao erro (b).

De acordo com CHARRABARTI (1962), GILL (1978) considerau aue o erro experimental para parcelas, e da se- 
guinte natureza $[1+(b-1) p] \approx^{2}$ e, para subparcelas $(1-p) \sigma^{2}$, com todas as covariancias nulas.

o seguinte quadro da analise da variancia fol apresentado por GILL (1978), con as respectivas esperancas dos quadrados medios, mostrando a correlacão constante entre duas subparcelas de uma mesma parcela.

\begin{tabular}{|c|c|c|}
\hline Causas de Variaçao & G.L. & $E(Q . M)$. \\
\hline Tratamento (A) & $a-1$ & $\sigma^{2}[1+(b-1) \rho]+b r \sum_{i=1}^{\alpha} \alpha_{i}^{2} / \theta_{A}$ \\
\hline Indiv/A (erro a) & $a(x-1)$ & $\sigma^{2}[1+(b-1) p]$ \\
\hline Entre Individuos & ar -1 & $---1---1------1$ \\
\hline Tempo ou Espaco (B) & $b-1$ & $\sigma^{2}(1-\rho)+\theta_{D B}^{2}+a \sum_{k=1}^{b} \beta_{k}^{2} / \theta_{B}$ \\
\hline$A \times B$ & $(a-1)(b-1)$ & $\sigma^{2}(1-\rho)+\sigma_{D B}^{2}+1 \sum_{i} \sum_{k}(\alpha \beta)_{i k}^{2} / \vartheta_{A B}$ \\
\hline Ind $/ A \times B=\operatorname{Erro}(b)$ & $a(r-1)(b-1)$ & $\sigma^{2}(1-\rho)+\alpha_{D B}^{2}$ \\
\hline Dentro de Indiv. & $\operatorname{ar}(b-1)$ & \\
\hline
\end{tabular}

Quanto aos testes de hipoteses, o autor considerou as seguintes hipoteses e seus respectivos testes: i) Interacaso, $H_{0}:(\alpha \beta)_{i k}=0$ para tado i, $\mathrm{k}$ Rejeita-se $H_{0}$ se $F=Q M_{A B} / Q M_{E} \geq F[\alpha ;(a-1)(b-1) ; \dot{a}(r-1)(b-1)]$. ii) fator $B, H_{0}: \beta_{k}=0$, parg todo $k$ Rejeita $H_{0}$ se $F=Q M_{B} / Q M_{E} \geq F[\alpha ;(b-1) ; a(x-1)(b-1)]$. iii) Fator $A, H_{a}: \alpha_{i}=0$, para todo $i$ Rejeita-se $H_{0}$ se $F=Q M_{A} / Q M_{D / A} \geq F[a ;(a-1) ; a(r-1)]$.

IEMMA (1981), considerou um experimento em parcelas subdivididas no qual os $v$ tratamentos principais estivessem presentes em $r$ dos a blocos incompletos equilibrados $(r<a)$; onde cada bloco era formado por $k$ parcelas e cada par de tratamentos principais ocorresse em $\lambda$ 
blocos. Considerou, também, a existencia de correlaça constante ( $(0)$, entre duas subparcelas de uma mesma parcela, e independéncia entre subparcelas de parcelas distintas.

Sob essas condiçres e através do método dos mínimos quadrados, o autor determinou: o sistema de equaços normais, as matrizes de dispersão para os parametros, os componentes de variancia para os efeitos fixos e aleatórios do modelo e os critérios para os testes das hipóteses de nulidade usuais e para comparaç̧es múltiplas pelo teste de Tukey.

Para verificar a mortalidade de Aphis rumicis MOORE \& BLISS (1942), estudaram sete inseticidas de contato, com os tratamentos aplicados nas trés primeiras linhas de um quadrado latino $7 \times 7$. Essas linhas foram consideradas como um experimento em bocos incompletos balanceados com trés repetiços, e em cada parcela form aplicados quatro concentraçres dos inseticidas.

Um exemplo foi apresentado por KEMPTHORNE (1947), de um experimento em reticulado quadrado com 25 tratamentos, na cultura da batata; os tratamentos foram constituidos por 22 materiais organicos e 3 testemunhas (um tratamento controle, representado trés vezes. Os 22 tratamentos organicos foram: cinco tipos de adubos, quatro materiais contendo sedimentos de esgoto e dois outros materiais organicos; sendo que cada um deles foi testado em dois niveis de aplicaşa. Cada parcela foi dividida em quatro subparcelas, onde foram alocadas combinaçzes de sulfato de amonia (N), superfosfato (P) e muriato de potássio ( $K)$. 
28.

\section{METODOLOGTA}

\subsection{Caracterizaço}

A metodologia a ser aplicada tem por finalidade a obtença da analise de dados, abtidos de um experimento em parcelas subdivididas, com os tratamentos principais dispostos segundo um delineamento em blocos incompletos parcialmente balanceados (PBIB).

Sem perda de generalidade, pode-se mostrax um delineamento em blocos incompletos parcialmente balanceados, do tipo reticulado quadrado com guatro tratamentos, no esquema de parcelas subdivididas quando os tratamentos principais estão dispostos em PBIB, atraves do seguinte esquema experimental não casualizad :

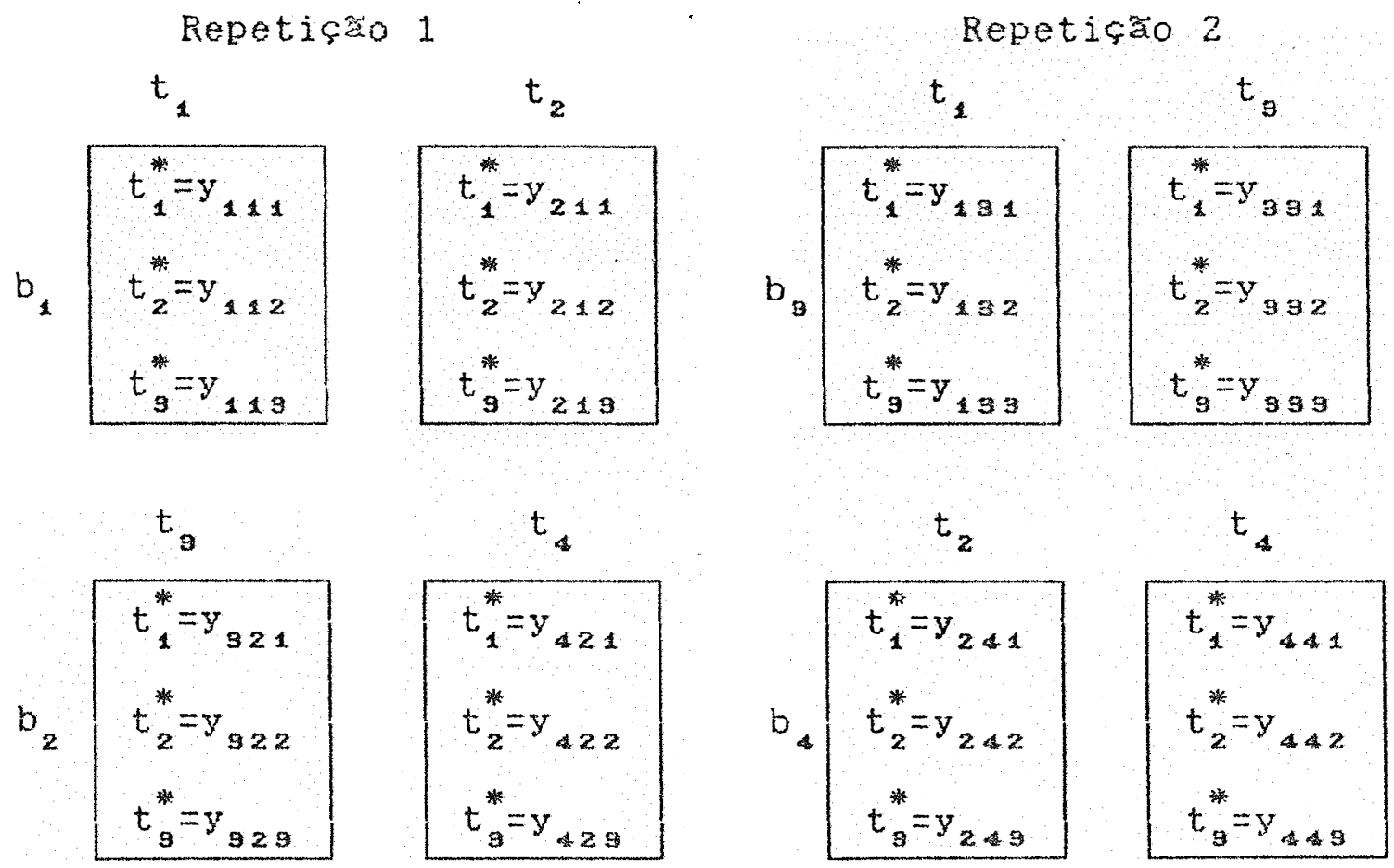

A ixaça dos três tratamentos secundários nas subpaxcelas tem apenas a finalidade didatica de apresentar o 
exemplo e dar uma melhor visualizaça do esquema experimental; na realidade eles devem ser casualizados dentro de cada parcela. No exemplo, tem-se: $v=4$ tratamentos principais $\left(t_{1}, t_{2}, t_{3}, t_{4}\right) ; b=4 \operatorname{blocos}\left(b_{1}, b_{2}, b_{3}, b_{4}\right) ; k=2$ tratamentos principais por bloco; $r=2$ repetiçes; $u=3$ tratamentos secundarios $\left(t_{1}^{*}, t_{2}^{*}, t_{3}^{*}\right)$ aplicados em cada tratamento principal. Um exemplo desse tipo de experimento, $a \quad 0$ caso citado por MIRANDA EILHO et alii (1986), cujo objetivo foi avaliar o potencial de populaçes de milho, onde nas parcelas foram alocadas as diferentes populaçes em um delineamento $P B I B$ e os cruzamentos reciprocos nas subparcelas.

\subsection{Um modelo Iinear}

Para o desenvolvimento da metodologia, adotouse o seguinte modelo linear matemático:

$$
y_{i j l}=\mu+b_{j}+t_{i}+t_{l}+\delta_{i l}+\delta_{i j l}
$$

com: $1=1,2, \ldots$ v tratamentos principais;

$\mathrm{j}=1,2, \ldots$ b blocos;

$1=1,2, \ldots$ u tratamentos secundários.

onde: $y_{i j l} e$ o valor observado na subparcela correspondente ao l-esimo tratamento secundario, dentro do i-esimo tratamento principal, no j-esimo bloco;

$\mu \quad$ e uma constante que representa a media geral;

$b_{j}$ e o efeito do j-ésimo bloco;

$t_{i}$ e ofeito do i-esimo tratamento principal;

$t_{i}$ e efeito do l-esimo tratamento secundario;

$S_{\text {il }} e$ efeito da interaç̃o entre o i-esimo tratamento principal e o l-ésimo tratamento secundario;

$*_{i j l} \quad$ o erro aleaterio atribuido a observacão $y_{i j l}$, considerado como o componente do residuo.

Na forma matrinial; o modelo linear e dado 
por :

$$
\mathrm{y}=\mathrm{X} \theta+\varepsilon
$$

onde: y un vetor de realizaçoes de variáveis aleatórias, com dimensoes (vru) $\times(1)$;

$X$ e uma matriz dos coeficientes dos parametros do modelo (matriz do delineamento), com dimensoes (vru) $x(1+v+b+u+v u)$;

- $\varepsilon$ um vetor de parametros descontecidos do modelo, com dimensoes $(1+v+b+u+v u) \times(1)$;

c 6 um vetor de variáveis aleatórias não observáveis, com dimensరes (vru) $x$ (1), as quais são assumidas serem independentes e normalmente distribuidas com $\varepsilon \sim N(\phi, \Sigma)$, cuja estrutura de erros $\varepsilon$ dada por:

$$
\operatorname{Cov}\left(y_{i j l} ; y_{i, j l}\right)= \begin{cases}\sigma_{a}^{2}+\sigma_{b}^{2}, & \text { se } i=i, j=j \text { e } 1=1 \\ \sigma_{a}^{2}, & \text { se } i=i, j=j \text { e } 1 \neq 1 \\ 0 & \text { em caso contrário } \quad(a .3)\end{cases}
$$

Conforme PIMENTEL GOMES (1968) e IEMMA (1981), efetuou-se a partiçăo da matriz $X$, da seguinte maneira:

$$
X=\left[X_{2}: X_{2}: x_{3}\left|X_{4}\right| X_{5}\right]
$$

onde:

$\mathrm{X}_{1}$ e o vetor dos coeficientes associados a constante $\mu$, de dimensðes (vru) $x$ (1);

$X_{2} E$ a matriz dos coeficientes associados aos blocos, de dimensoes (vru) $x$ (b);

$\mathrm{X}_{\mathrm{a}}$ E a matriz dos coeficientes associados aos tratamentos principais, de dimensoes (vru) x (v);

$X_{4}$ e a matriz dos coeficientes associados aos tratamentos secundários, de dimensoes (vru) x (u);

$x_{5}$ E a matriz dos coeficientes assooiados às interaç̧es $\left(t t^{\prime}\right)_{i l}$ ou $\delta_{i l}$, de dimensठ̋s (vru) x (vu).

A partição do vetor dos parametros $\theta$ correspondente à partiçăo da matriz X é dada por:

$$
\theta^{\prime}=\left[\mu \mid \beta^{\prime}: \tau^{\prime} \tau^{*}, \delta^{\prime}\right]
$$

onde, 


$$
\beta=\left[\begin{array}{l}
n_{1} \\
b_{2} \\
\cdots \\
b_{v}
\end{array}\right]_{1} \tau=\left[\begin{array}{c}
t_{1} \\
t_{2} \\
\cdots \\
t_{v}
\end{array}\right]_{1} ; \tau^{*}=\left[\begin{array}{c}
t_{1}^{*} \\
t_{2}^{*} \\
t_{u}^{*}
\end{array}\right]_{1} \delta=\left[\begin{array}{c}
\delta_{11} \\
\delta_{12} \\
\sigma_{v u}
\end{array}\right]_{1}
$$

Considerou-se a năo existência de uma correlaç̃o entre as subparcelas de uma mesma parcela e a independência entre subparcelas de parcelas distintas.

3.3. Sistema de equacbos normais

Aplicando o método dos minimos quadrados, determinou-se o seguinte sistema de equaçes normais (SEN):

$$
x^{\prime} x \theta=x^{\prime} y
$$

o qual resultou en

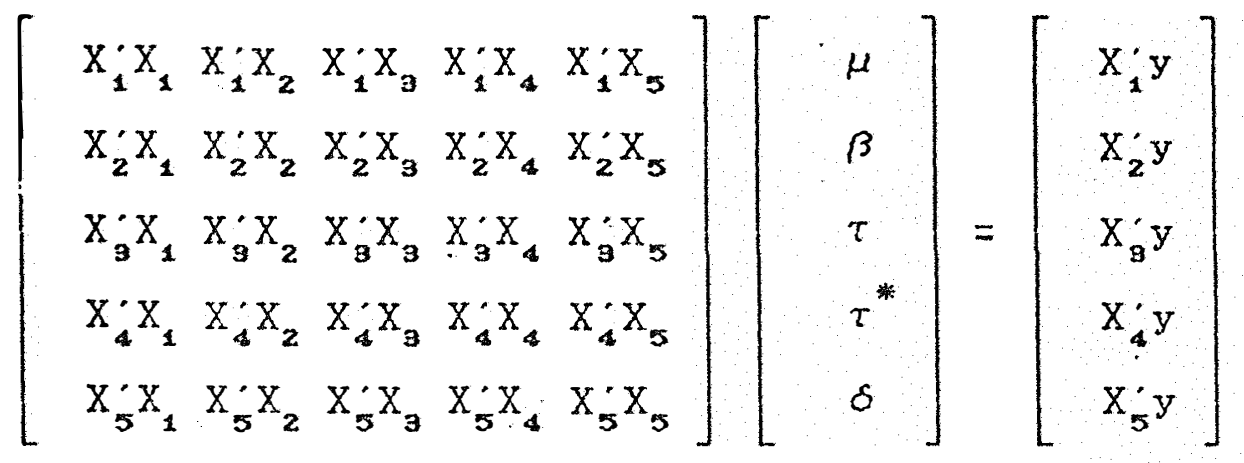

onde as submatrizes são designadas por:

$x_{1}^{\prime} x_{1}$ E uma submatriz, de dimensöes (1) $x$ (1), constituida por bku $=$ vru $=n$ que $e$ o número total de unidades experimentais ou de subparcelas;

$\mathrm{X}_{1} \mathrm{X}_{2}$ e uma submatriz, de dimensठies (1) $\mathrm{x}$ (b), representada por $K=k u E_{b}$, correspondente ao vetor associado ao número de subparcelas en cada bloco, cujo $E$ e um vetor de uns;

$\mathrm{X}_{1}^{\prime} \mathrm{X}_{3} \Leftrightarrow$ uma submatriz, de dimensões (1) $x(v)$, representada 
por $R=r u{ }_{i} E_{v}$, correspondente ao vetor associado ao número de repetiçชes dos tratamentos principais, cujo $E$ é um vetor de uns;

$\mathrm{X}_{1}^{\prime} \mathrm{X}_{4}$ é uma submatriz, de dimensðes ( 1 ) $\mathrm{x}$ ( $u$ ), representada por $U=\operatorname{vr} E_{1}$, correspondente ao vetor associado ao número de repetiçðes dos tratamentos secundários, cujo E é um vetor de uns;

$\mathrm{X}_{1}^{\prime} \mathrm{X}_{5}$ é uma submatriz, de dinenszes (1) $\mathrm{x}$ (vu), representada por $L=r E_{v u}$, correspondente ao vetor associado ao número de repetiços das interaçoes, cujo $E$ é um vetor de uns;

$\mathrm{X}_{2} \mathrm{X}_{2}$ é uma submatriz diagonal $\{k u, k u, \ldots, k u\}$, de dimensठes (b) $x(b)$, representada por $R$, correspondente a matriz do número de subparcelas em cada bloco;

$\mathrm{X}_{2} \mathrm{X}_{4}$ e uma submatriz, de dimensoes (b) $x$ ( $u$ ), representada por $A=k E$, correspondente a matriz do número de tratamentos principais por bloco, cujo $E \&$ uma submatriz de uns;

$\mathrm{X}_{2} \mathrm{X}_{5}$ é uma submatriz, de dimensठes (b) $x$ (vu), representada por $V$, correspondente a matriz de incidência da interação (tt) $i l$ no bloco $j$, constituida por $v_{i, i l}$, onde:

$$
v_{j, i l}=\left\{\begin{array}{l}
1, \text { se a interaçao }\left(t t^{\prime}\right)_{i l} \text { ocorre no bloco } j, \\
0, \text { caso contráxio; }
\end{array}\right.
$$

$\mathrm{X}_{\mathbf{a}}^{\prime} \mathrm{X}_{\mathbf{2}}$ é uma submatriz, de dimenszes ( $v$ ) $\mathrm{x}$ (b), correspondente a matriz de incidência do i-ésimo tratamento principal no j-ésimo bloco, denominada $N$, cujos elementos que a compoem så $\mathrm{n}_{i j}$, onde:

$n_{i j}=\left\{\begin{array}{l}u, \text { se o tratamento principal } i \text { ocorre no bloco } \mathrm{j} ; \\ 0, \text { caso contrário: }\end{array}\right.$

$\mathrm{X}_{3} \mathrm{X}_{3}$ \& uma submatriz diagonal $\{\mathrm{ku}, \mathrm{ku}, \ldots, \mathrm{ku}\}$, de dimenşes (v) $x(v)$, representada por $R$, correspondente ao número do repetisoes de cada tmatamento principal; 
$\mathrm{X}_{3}^{\prime} \mathrm{X}_{4}$ ó uma submatriz, de dimensoes (v) $x(u)$, correspondente ao número de repetiçães de cada par $\left(t t^{\prime}\right)_{i l}$ da interaça, representada por $P=r \cdot{ }_{v}{ }_{u}$, cujo $E \Leftarrow$ uma submatriz de uns;

$\mathrm{X}_{3}^{\prime} \mathrm{X}_{5} \&$ uma submatriz, de dimensठes (v) $\mathrm{x}$ (vu), representada por $S$, corres[ondente a matriz de incidencia dos tratamentos principais nos pares $\left(t t^{\prime}\right)_{i l}$, onde:

$$
s_{i, i l}=\left\{\begin{array}{l}
r, \begin{array}{l}
\text { se a combinasão }\left(t t^{\prime}\right)_{i l} \text { o c orre no tr a tamento } \\
\text { principal 1; }
\end{array} \\
0, \text { caso contrário: }
\end{array}\right.
$$

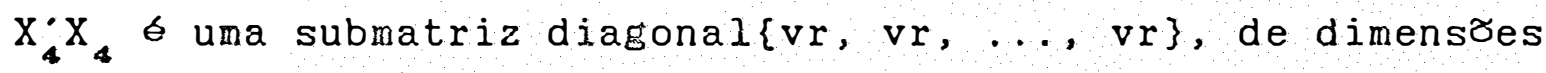
(u) $x(u)$, correspondente ao número de repetiçes dos tratamentos secundários, representada por $U$;

$\mathrm{X}_{4}^{\prime} \mathrm{X}_{5}$ é uma submatriz, de dimensठes (u) $\mathrm{x}$ (vu), representada por $H=\left[\begin{array}{l|l|l|l}r I_{(u)} & r I_{(u)} & \ldots & r I_{(u)}\end{array}\right]$, e correspondente a matriz de incidência dos tratamentos secundarios nos pares $\left(t t^{\prime}\right)_{i l}$, cujo $I \subseteq$ uma matriz identidade de dimenses $(u) x(u)$;

$X_{5}^{\prime} X_{5} 6$ uma submatriz diagonal $\{r, r, \ldots, r\}$, de dimens (vu) $x$ (vu), associada ao número de repetiçбes dos pares $\left(t t^{\prime}\right)_{i l} d a$ interaçă, representada por L;

$x_{1}^{\prime} y$ ó um vetor referente ao total geral observado, de dimensaes (1) $x(1)$, representado por $G$;

$\mathrm{X}_{2}^{\prime} \mathrm{y}$ é um vetor com os totais de blocos, de dimensoes (b) $x$ (1), representado por $B$, sendo $B^{\prime}=\left[B_{1}, B_{2}, \ldots, B_{b}\right]$;

$X_{a}^{\prime} y \circlearrowleft$ um vetor com os totais de tratamentos principais, de dimensठes ( $v$ ) $x$ ( 1 ), representado por $T$, sendo $\mathrm{T}^{\prime}=\left[\mathrm{T}_{1}, \mathrm{~T}_{2}, \ldots, \mathrm{T}_{\mathrm{v}}\right]$

$\mathrm{X}_{4}^{\prime} \mathrm{y} \Leftrightarrow$ um vetor com os totais de tratamentos secundarios, de dimenszes ( $u$ ) $x(1)$, representado por $\mathrm{T}^{*}$, sendo $\mathrm{T}^{*}=\left[\mathrm{T}_{1}^{*}, \mathrm{~T}_{2}^{*}, \ldots, \mathrm{T}_{\mathrm{u}}^{*}\right]$;

$x_{5}^{\prime} y \Leftrightarrow u m$ vetor com os totais de interacão, de dimensces (vu) $x$ (1), representado por $D$, sendo 
34.

$$
D^{\prime}=\left[\mathrm{TT}_{11}^{*}, \ldots, \mathrm{TT}_{1 \mathrm{u}}^{*}, \ldots, \mathrm{TT}_{\mathrm{V} 1}^{*}, \ldots, \mathrm{TT}_{\mathrm{Vu}}^{*}\right] \text {. }
$$

Desse modo, as partiçes efetuadas em (a.5), estao escritas na seguinte forma:

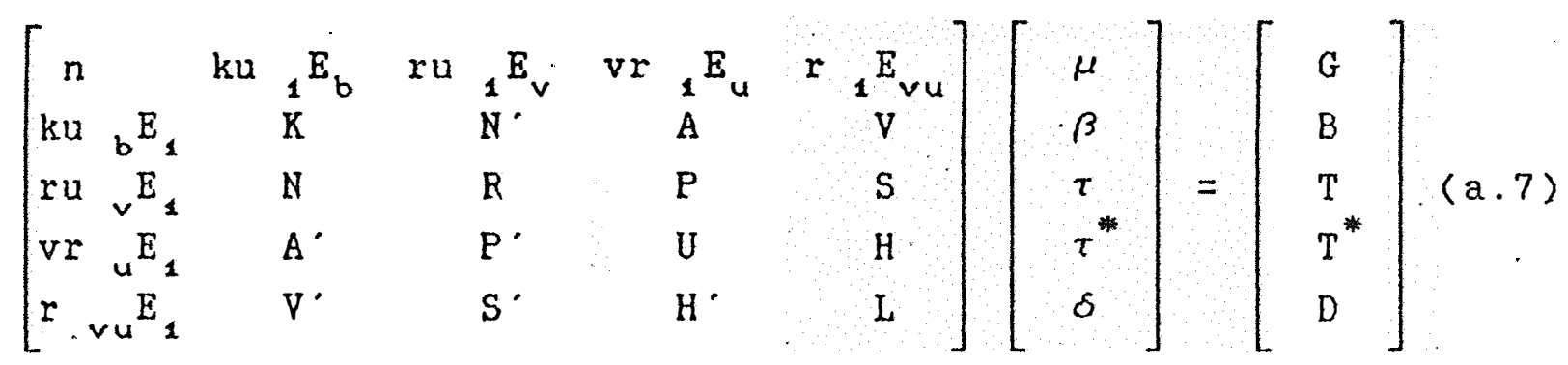

Efetuando-se as multiplicaçres sugeridas em (a.6), o sistema de equaços normais pode ser escrito do seguinte modo

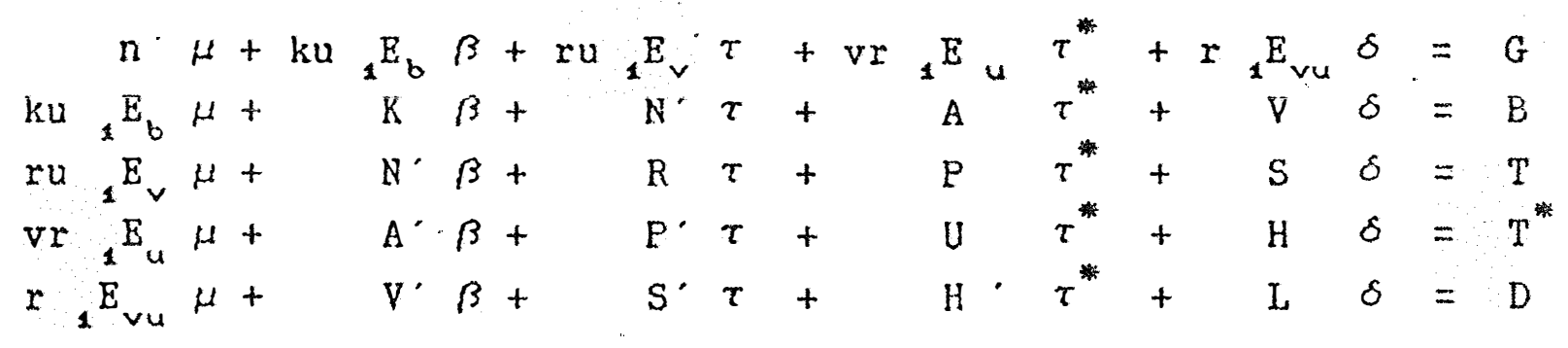

\subsection{Solucao do sistema de equaçes normais}

- número de equaç⿸es do sistema de equaçres normais em $(a .5) \Leftrightarrow 1+b+v+u+v u$; mas, verifica-se que a soma das equaçres $\beta$, a soma das equaçðes $\tau$ e a soma das equaçres $\tau^{*}$ são idênticas à equação $\mu$, implicando em três relaçరes linearmente dependentes entre as linhas de $X ' X$. Nas equaços $\hat{o}$, as somas em relaça a $\mathrm{k}$, são iguais às equaços $\tau$ para $i=1, \ldots, v$ representando outras $v$ relaçđes iineares entre as linhas de $X^{\prime} X$. Do mesmo modo, as somas em relação a $i$, são iguais às equaçæes $\tau^{*}$ para $1=1, \ldots, u$; entretanto, dessas u relaçzes lineares representadas agora, somente u-1 delas são linearmente independentes daquelas já descritas. Portanto, o número de relaças lineares dependentes e $3+v+u-1=v+u+2$.

Assin, 0 posto de $X^{\prime} X$ é $r[X]=v u+b-1 \leq \min \{n, p\}$, sendo $a$ o número de partametros, entzo as equaczes normais de 
(a.5) năo tem soluçăo única. Pois, $X^{\prime} X$ é singular, $e$ portanto não existe $\left(X^{\prime} X\right)^{-1}$ e o SEN é indeterminado, mas é sempre consistente (IEMMA, 1888).

Uma solução de minimos quadrados é $e^{\circ}=\left(X^{\prime} X\right)^{\circ} X^{\prime} y$, para qualquer inversa generalizada de $X^{\prime} X$. Uma outra alternativa a estratégia de "completar" o posto da matriz $X$. Isto tem sido feito de vários modos; dentre êles, cita-se a restriçăo nas soluçðes. A restriçăo tem como objetivo completar o posto da matriz $X ' X$, tornando-a invertivel, e assim determinando um $\hat{\theta}$ único.

Então, completando-se o posto da matriz X, com $\mathrm{p}-\mathrm{r}[\mathrm{X}]=\mathrm{v}+\mathrm{u}+2$ linhas linearmente independentes das linhas da matriz $X$, que constituem um conjunto de funçres paramétricas conjuntamente não estimáveis e independentes entre si.

Un conjunto de restriçoes é formado pelas sequintes funçôes lineares năo estimáveis:

$$
\sum_{j=1}^{b} \hat{b}_{j}=\sum_{i=1}^{v} \hat{t}_{i}=\sum_{\substack{l=1 \\ \text { Ressalta-se que, se outro conjunto de }}}^{u} \hat{t}_{i=1}^{*}\left(\hat{\delta}_{i l}\right)=\sum_{i=1}^{u}\left(\hat{\delta}_{i l}\right)=0 \text { (a.8) }
$$
çres é adotado, obtém-se outra soluçăo, mas $X \theta^{\circ}$ é invariante para qualquer $\theta^{0}$ solusăo de (a.6), segundo IEMMA (1988).

\subsubsection{PBIB com $m$ classes de associados}

De acordo com as restriçres impostas em (a.8) e face às estruturas das matrizes envolvidas, o SEN resultou em

$$
\begin{aligned}
& n \hat{\mu}=\mathrm{G} \\
& \mathrm{ku}{ }_{b} \mathrm{E}_{1} \hat{\mu}+\mathrm{K} \hat{\beta}+\mathrm{N} \hat{\tau}=\mathrm{B} \quad(\mathrm{a} .10) \\
& \mathrm{ru} \mathrm{v}_{1} \hat{\mu}+\mathrm{N} \hat{\beta}+\mathrm{R} \hat{\tau} \quad=\mathrm{T} \text { (a.11) } \\
& \text { vr }{ }_{4}{ }_{1} \hat{\mu}+\hat{U \tau}^{*}+\mathrm{T}^{*} \quad(\mathrm{a} .12) \\
& r_{V r} E_{1} \hat{\mu}+V^{\prime} \hat{\beta}+S^{\prime} \hat{\tau}+H^{\prime} \hat{\tau}^{*}+L^{\hat{\delta}}=D \text { (a.13) }
\end{aligned}
$$

gue resulta em 


$$
\begin{aligned}
n \hat{\mu} & =G \\
\left(K-N R^{-1} N\right) \hat{\beta} & =\left(B-N R^{-1} T\right) \\
\left(R-N K^{-1} N\right) \hat{\tau} & =\left(T-N K^{-1} B\right) \\
U 1 \hat{\mu}+U \hat{\tau}^{*} & =T^{*} \\
L 1 \hat{\mu}+V^{\prime} \hat{\beta}+S^{\prime} \hat{\tau}+H^{\prime} \hat{\tau}^{*}+L \hat{\delta} & =D
\end{aligned}
$$

onde (a.16) representa o sistema de equacres normais reduzido (SENR) ou sistema de equaças normais para efeitos ajustados de tratamentos principais.

Resultado analogo pode-se obter, pois o principal interesse $\&$ o efeito dos tratamentos, eliminando-se os efeitos de $\hat{\mu}$ e de $\hat{\beta} \in m(a .10)$ e (a.11), ou pre-multiplicando o SEN de (a.7) por:

$$
\left[\begin{array}{ccccc}
1 & 0 & 0 & \varnothing & 0 \\
0 & I_{b} & -N^{\prime} R^{-1} & 0 & 0 \\
\theta & -N K^{-1} & I_{v} & 0 & 0 \\
0 & \varnothing & 0 & I_{u} & 0 \\
0 & \varnothing & \varnothing & 0 & I_{v u}
\end{array}\right]
$$

\subsubsection{Estimaço dos efeitos de tratamentos principais}

Da teoria geral de blocos incompletos, discutida por PIMENTEI GOMES (1968), JOHN (1980), dentre outros, pode-se escrever o SENR de (a.16) na forma:

$$
\text { C } \hat{\tau}=Q
$$

onde,

$$
C=R-N K^{-1} N^{-}
$$

E uma matriz de dimensơs ( $v$ ) $x(v)$, com caracteristica $v-1$, e com os seguintes elementos:

$$
c_{i i^{\prime}}=\left\{\begin{array}{ll}
r u(k-1) / k & , \text { se } i=i^{\prime} \\
-u \lambda_{i i}, / k, & \text { se } i \neq i
\end{array}, i, i^{\prime}=1,2, \ldots, v\right.
$$

sendo $\lambda_{i i}$ o número de vezes que os tratamentos principais $i$ $e i$. ocorrem juntos no mesmo bloco. 


$$
Q=T-N K^{-1} B
$$

6 o vetor de totais ajustados de tratamentos principais, e 6 composto pelos sequintes elementos:

$$
Q_{i}=T_{i}-A_{i} / k
$$

sendo $A_{i}$ a soma dos totais dos blocos que contem o tratamento principal i.

Dado que a matriz C é singular, o SENR possui infinitas soluçశes. Uma solução de minimos quadrados, pode ser a utilização de inversas generalizadas (RAO, 1973; JOHN, 1980; IEMMA, 1988), ou introduzindo-se restriçбes (PIMENTEL GOMES, 1968; IEMMA, 1981; OLIVEIRA, 1985; DAS \& GIRI, 1986). De acordo com o SENR $C \hat{\tau}=Q$, e conforme a caracterização da matriz C, dada em (a.19), para um dado tratamento principal s tem-se a equaça seguinte equaça normal reduzida para $\tau$ :

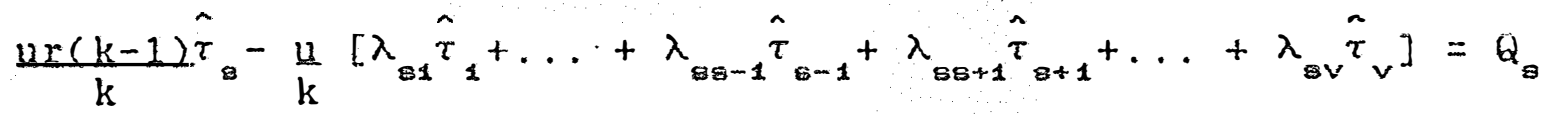
sendo $s=1,2, \ldots, v$ tratamentos principais; ou

$$
u r(k-1) \hat{\tau}_{8}-u \sum_{i=1}^{m} \lambda_{i} S_{i}\left(\hat{\tau}_{8}\right)=k Q_{8}
$$

onde $S_{i}\left(\hat{\tau}_{s}\right)$ corresponde a soma dos efeitos dos tratamentos principais que são os $i-e s i m o s$ associad so tratamento $s$, e $\lambda_{i}$ \& um dos parsmetros do primeiro tipo, definido por BOSE \& NAIR (1939), com $i=1,2, \ldots, m$ associados.

Verifica-se, que em (a.24), o efeito do tratamento principal $\hat{\tau}_{s}$ na forma como é colocado, está em funçăo de seus associados, que tambem não são conhecidos.

Segundo CHAKRABARTI (1962), se um conjunto de tratamentos obedece à uma estrutura de PBIB, então as seguintes igualdades se verificam:

$$
S_{j} S_{i}\left(\hat{\tau}_{s}\right)= \begin{cases}\sum_{j=1}^{m} p_{i j}^{i} S_{j}\left(\hat{\tau}_{s}\right) & \text { se } j \neq t_{i} \\ n_{i} \tau_{s}+\sum_{j=1}^{m} p_{i}^{j} S_{j}\left(\hat{\tau}_{s}\right), & \text { se } j=i\end{cases}
$$


38.

Assim, a equação (a.24), usando o procedimento de CHAKRABARTI (1962), pode ser escrita como:

$$
\mathrm{kS}_{j^{\prime}}\left(Q_{B}\right)=u r(k-1) S_{j^{\prime}}\left(\hat{\tau}_{\theta}\right)-u \sum_{i=1}^{m} \lambda_{i} S_{j^{\prime}} S_{i}\left(\hat{\tau}_{B}\right)
$$

onde $S_{j}\left(Q_{s}\right)$ representa a soma dos $Q^{\prime} s$ dos $j^{\prime}$-ésimos associados do s-ósimo tratamento principal:

a) Para $j^{\prime}=1$, a equação (a.26) fica como:

$$
k S_{i}\left(Q_{B}\right)=u r(k-1) S_{1}\left(\hat{\tau}_{B}\right)-u \sum_{i=1}^{m} \lambda_{i} S_{1} S_{i}\left(\hat{\tau}_{B}\right)
$$

onde,

$$
\begin{aligned}
& \sum_{i=1}^{m} \lambda_{i} S_{i} S_{i}\left(\hat{\tau}_{s}\right)=\lambda_{1} S_{1} S_{1}\left(\hat{\tau}_{8}\right)+\lambda_{2} S_{i} S_{2}\left(\hat{\tau}_{8}\right)+\ldots+\lambda_{m} S_{1} S_{m}\left(\hat{\tau}_{s}\right) \\
& \text { Usando as igualdades de (a.25), tem-se } \\
& \sum_{i=1}^{m} \lambda_{i} S_{1} S_{i}\left(\hat{\tau}_{B}\right)=\lambda_{1}\left[n_{1} \hat{\tau}_{\theta}+p_{11}^{1} S_{\alpha}\left(\hat{\tau}_{\theta}\right)+p_{11}^{2} S_{2}\left(\hat{\tau}_{\theta}\right)+\ldots+p_{11}^{m} S_{m}\left(\hat{\tau}_{\theta}\right)\right]+ \\
& +\lambda_{2}\left[p_{12}^{1} \mathrm{~S}_{1}\left(\hat{\tau}_{\theta}\right)+\mathrm{p}_{12}^{2} \mathrm{~S}_{2}\left(\hat{\tau}_{8}\right)+\ldots+\mathrm{p}_{12}^{\mathrm{m}} \mathrm{S}_{\mathrm{m}}\left(\hat{\tau}_{s}\right)\right]+ \\
& +\ldots \ldots \ldots \ldots \ldots \ldots \ldots \ldots \ldots \ldots \ldots \ldots \ldots \ldots \ldots \ldots \ldots \ldots \ldots \ldots+ \\
& +\lambda_{m}\left[p_{1 m}^{1} S_{1}\left(\hat{\tau}_{s}\right)+p_{1 m}^{2} S_{2}\left(\hat{\tau}_{s}\right)+\ldots+p_{1 m}^{m} S_{m}\left(\hat{\tau}_{s}\right)\right] \\
& \text { Mas, sabe-se por BOSE \& NAIR (1939), que: } \\
& \sum_{j=1}^{m} p_{j j}^{i}= \begin{cases}n_{j}-1, & \text { se } j=i \\
n_{j}, & \text { se } j \neq i\end{cases}
\end{aligned}
$$
anterior, tem-se que:

$$
\begin{aligned}
& \sum_{i=1}^{m} \lambda_{i} S_{1} S_{i}\left(\hat{\tau}_{s}\right)=\lambda_{1}\left[n_{1} \hat{\tau}_{8}+\left(n_{1}-1-p_{12}^{1}-\cdots-p_{1 m}^{1}\right) S_{1}\left(\hat{\tau}_{8}\right)+\right. \\
& +\left(n_{1}-p_{12}^{2}-\cdots-p_{1 m}^{2}\right) s_{2}\left(\tilde{\tau}_{s}\right)+\ldots+ \\
& +\left(n-p_{1}^{m} \cdots-p m_{1 m}^{m} S(\hat{\tau})\right]+ \\
& +\lambda_{2}\left[p_{12}^{1} S_{1}\left(\hat{\tau}_{s}\right)+p_{12}^{z} S_{2}\left(\hat{\tau}_{g}\right)+\ldots+p_{12}^{m} S_{m}\left(\hat{\tau}_{s}\right)\right]+
\end{aligned}
$$

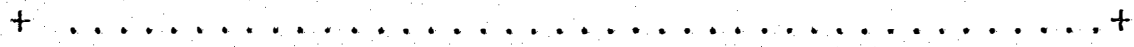

$$
\begin{aligned}
& +\lambda_{m}\left[p_{1 m}^{1} S_{1}\left(\hat{\tau}_{8}\right)+p_{1 m}^{2} S_{2}\left(\hat{\tau}_{8}\right)+\cdots+p_{1 m}^{m} S_{m}\left(\hat{\tau}_{8}\right)\right]
\end{aligned}
$$




$$
\begin{aligned}
& \sum_{i=1}^{m} \lambda_{i} S_{1} S_{i}\left(\hat{\tau}_{s}\right)=\lambda_{1}\left[n_{1}\left[\hat{\tau}_{s}+S_{1}(\hat{\tau})+S_{2}\left(\hat{\tau}_{s}\right)+\ldots+s_{m}\left(\hat{\tau}_{s}\right)\right]+\right. \\
& -\left(1+p_{12}^{1}-\cdots-p_{1 m}^{1}\right) s_{1}\left(\hat{\tau}_{s}\right)-\ldots- \\
& \left.-\left(p_{12}^{m}-\cdots-p_{1 m}^{m}\right) s_{m}\left(\hat{\tau}_{s}\right)\right\}+ \\
& +\lambda_{2}\left[p_{12}^{1} S_{1}\left(\hat{\tau}_{\theta}\right)+p_{12}^{2} S_{2}\left(\hat{\tau}_{\theta}\right)+\ldots+p_{12}^{m} S_{m}\left(\hat{\tau}_{\theta}\right)\right]+ \\
& +\ldots \ldots \ldots \ldots \ldots \ldots \ldots \ldots \ldots \ldots \ldots+ \\
& +\lambda_{m}\left[p_{1 m}^{1} S_{1}\left(\hat{\tau}_{8}\right)+p_{1 m}^{2} S_{2}\left(\hat{\tau}_{8}\right)+\ldots+p_{1 m}^{m} S_{m}\left(\hat{\tau}_{8}\right)\right]
\end{aligned}
$$

Utilizando-se a restriça

$$
\hat{\tau}_{B}+S_{1}\left(\hat{\tau}_{\theta}\right)+S_{2}\left(\hat{\tau}_{s}\right)+\ldots+S_{m}\left(\hat{\tau}_{s}\right)=0
$$

abtem-se:

$$
\begin{aligned}
& \sum_{i=1}^{m} \lambda_{i} S_{1} S_{i}\left(\hat{\tau}_{s}\right)=-\lambda_{1}\left[\left(n_{1}-p_{11}^{1}\right) S_{1}\left(\hat{\tau}_{3}\right)+\left(n_{1}-p_{11}^{2}\right) S_{2}\left(\hat{\tau}_{3}\right)+\ldots\right. \\
& \left.\cdots+\left(n_{1}-p_{11}^{m}\right) S_{m}\left(\hat{\tau}_{s}\right)\right]+ \\
& +\lambda_{2}\left[p_{12}^{1} S_{1}\left(\hat{r}_{s}\right)+p_{12}^{2} S_{2}\left(\hat{\tau}_{s}\right)+\ldots+p_{12}^{m} S_{m}\left(\hat{\tau}_{s}\right)\right]+ \\
& +\ldots \ldots \ldots \ldots \ldots \ldots \ldots \ldots \ldots \ldots \ldots \ldots \ldots \ldots \ldots \ldots \ldots \ldots \ldots+ \\
& +\lambda_{m}\left[p_{1 m}^{1} S_{1}\left(\hat{\tau}_{s}\right)+p_{1 m}^{2} S_{2}\left(\hat{\tau}_{8}\right)+\ldots+p_{1 m}^{m} S_{m}\left(\hat{\tau}_{s}\right)\right] \\
& =-\left[\lambda_{1} n_{1}-\lambda_{1} p_{11}^{1}-\lambda_{2} p_{12}^{1}-\ldots-\lambda_{m} p_{1 m}^{1}\right] S_{1}\left(\hat{\tau}_{\theta}\right)- \\
& -\left[\lambda_{1} n_{1}-\lambda_{1} p_{11}^{2}-\lambda_{2} p_{12}^{2}-\ldots-\lambda_{m} p_{1 m}^{2}\right] S_{2}\left(\hat{\tau}_{s}\right)-
\end{aligned}
$$

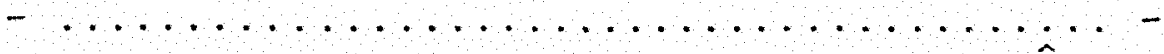

$$
\begin{aligned}
& -\left[\lambda_{1} n_{1}-\lambda_{1} p_{11}^{m}-\lambda_{2} p_{12}^{m}-\cdots-\lambda_{m} p_{1 m}^{m}\right] S_{m}\left(\hat{\tau}_{s}\right) \\
& \begin{aligned}
\sum_{i=1}^{m} \lambda_{i} S_{1} S_{i}\left(\hat{\tau}_{s}\right)= & -\left[\lambda_{1} n_{1}-\sum_{l=1}^{m} \lambda_{l} p_{1 l}^{l}\right] S_{1}\left(\hat{\tau}_{s}\right)- \\
& -\left[\lambda_{1} n_{1}-\sum_{l=1}^{m} \lambda_{l} p_{1 l}^{2}\right] S_{2}\left(\hat{\tau}_{s}\right)-\ldots . \\
& -\left[\lambda_{1} n_{1}-\sum_{l=1}^{m} \lambda_{l} p_{1 l}^{m}\right] S_{m}\left(\hat{\tau}_{s}\right) .
\end{aligned}
\end{aligned}
$$

Substituindo a expressão (a.30) em (a.27), obtem-se a seguinte equação: 


$$
\begin{aligned}
k S_{1}\left(Q_{s}\right)=u & {\left[r(k-1)+\lambda_{1} n_{1}-\sum_{l=1}^{m} \lambda_{l} p_{1 l}^{1}\right] S_{1}\left(\hat{\tau}_{8}\right)+} \\
& +u\left[\lambda_{1} n_{1}-\sum_{l=1}^{m} \lambda_{l} p_{1 l}^{2}\right] S_{2}\left(\hat{\tau}_{s}\right)+\cdots+ \\
& +u\left[\lambda_{1} n_{l}-\sum_{l=1}^{m} \lambda_{l} p_{1 l}^{m}\right] S_{m}\left(\hat{\tau}_{s}\right) .
\end{aligned}
$$

Procedendo-se desse modo para as demais expressoes relativas aos $\mathrm{j}$-osimos associados (restantes) de $\tau_{8}$, obtem-se:

b) Para $j=m$, a equaç̃o (a.26) fica como:

$$
\mathrm{kS}_{m}\left(Q_{s}\right)=u r(k-1) S_{m}\left(\hat{\tau}_{s}\right)-u \sum_{i=1}^{m} \lambda_{i} S_{m} S_{i}\left(\hat{\tau}_{s}\right)
$$

onde,

$$
\begin{aligned}
& \sum_{i=1}^{m} \lambda_{i} S_{m} S_{i}\left(\hat{\tau}_{s}\right)=\lambda_{1} S_{m} S_{1}\left(\hat{\tau}_{s}\right)+\lambda_{2} S_{m} S_{2}\left(\hat{\tau}_{s}\right)+\ldots+\lambda_{m} S_{m} S_{m}\left(\hat{\tau}_{s}\right) \\
& \text { Usando as igualdades de (a.25), tem-se } \\
& \sum_{i=1}^{m} \lambda_{i} S_{m} S_{i}\left(\hat{\tau}_{s}\right)=\lambda_{1}\left[p_{m 1}^{1} S_{1}\left(\hat{\tau}_{s}\right)+p_{m s}^{2} S_{2}\left(\hat{\tau}_{s}\right)+\ldots+p_{m 1}^{m} S_{m}\left(\hat{\tau}_{\theta}\right)\right]+ \\
& +\lambda_{2}\left[\mathrm{p}_{m 2}^{1} \mathrm{~S}_{1}\left(\hat{\tau}_{8}\right)+\mathrm{p}_{m 2}^{2} \mathrm{~S}_{2}\left(\hat{\tau}_{8}\right)+\ldots+\mathrm{p}_{m 2}^{m} \mathrm{~S}_{m}\left(\hat{\tau}_{8}\right)\right]+
\end{aligned}
$$

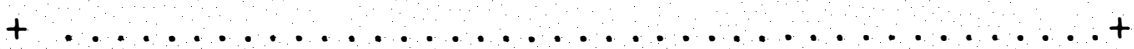

$$
\begin{aligned}
& +\lambda_{m}\left[n_{m} \hat{\tau}_{s}+p_{m m}^{1} S_{1}\left(\hat{\tau}_{s}\right)+p_{m m}^{2} S_{2}\left(\hat{\tau}_{s}\right)+\ldots+p_{m m}^{m} S_{m}\left(\hat{\tau}_{s}\right)\right] \\
& \text { Mas, de (a.28) tem-se que: }
\end{aligned}
$$

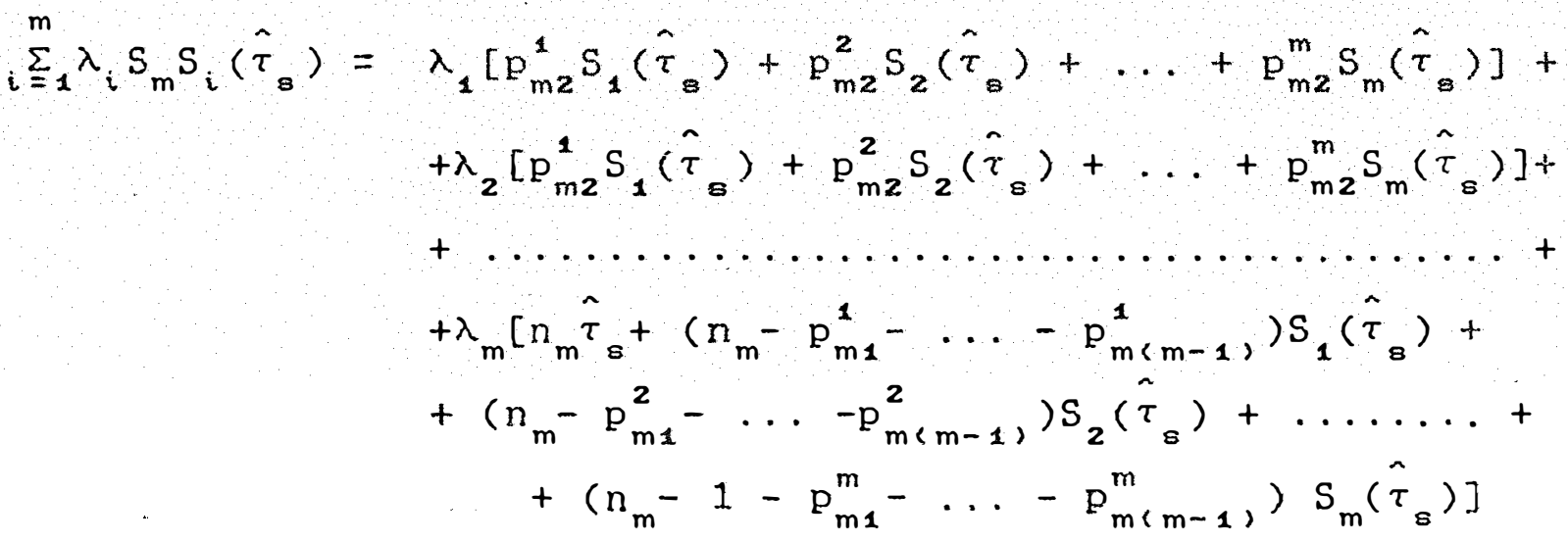




$$
\begin{aligned}
& \sum_{i=1}^{m} \lambda_{i} S_{m} S_{i}\left(\hat{\tau}_{s}\right)=\lambda_{1}\left[p_{m 1}^{1} S_{1}\left(\hat{\tau}_{s}\right)+p_{m 1}^{2} S_{2}\left(\hat{\tau}_{s}\right)+\cdots+p_{m 1}^{m} S_{m}\left(\hat{\tau}_{s}\right)\right]+ \\
& +\lambda_{2}\left[p_{m z}^{1} S_{1}\left(\hat{\tau}_{\theta}\right)+p_{m z}^{2} S_{2}\left(\hat{\tau}_{s}\right)+\cdots+p_{m z}^{m} S_{m}\left(\hat{\tau}_{g}\right)\right]+ \\
& +\ldots \ldots \ldots \ldots \ldots \ldots \ldots \ldots \ldots \ldots+ \\
& +\lambda_{m}\left\{n_{m}\left[\hat{\tau}_{s}+s_{1}\left(\hat{\tau}_{s}\right)+S_{2}\left(\hat{\tau}_{s}\right)+\ldots+S_{m}\left(\hat{\tau}_{s}\right)\right]-\right. \\
& -\left(n_{m}-p_{m m}^{1}\right) S_{1}\left(\hat{\tau}_{s}\right)-\left(n_{m}-p_{m m}^{2}\right) S_{2}\left(\hat{\tau}_{s}\right)- \\
& \left.\cdots \ldots-\left(n_{m}-p_{m m}^{m}\right) s_{m}\left(\hat{\tau}_{s}\right)\right\}
\end{aligned}
$$

Utilizando-se a restricao de (a.29), obten-se:

$$
\begin{aligned}
& \sum_{i=1}^{m} \lambda_{i} S_{m} S_{i}\left(\hat{\tau}_{s}\right)=\lambda_{1}\left[p_{m 1}^{1} S_{1}\left(\hat{\tau}_{s}\right)+p_{m 1}^{2} S_{2}\left(\hat{\tau}_{s}\right)+\cdots+p_{m 1}^{m} S_{m}\left(\hat{\tau}_{s}\right)\right]+ \\
& +\lambda_{2}\left[\mathrm{p}_{m z}^{1} \mathrm{~S}_{1}\left(\hat{\tau}_{g}\right)+\mathrm{p}_{m z}^{2} \mathrm{~S}_{2}\left(\hat{\tau}_{s}\right)+\ldots+\mathrm{p}_{m 2}^{m} \mathrm{~S}_{m}\left(\hat{\tau}_{\theta}\right)\right]+ \\
& +\ldots \ldots \ldots \ldots \ldots \ldots \ldots \ldots \ldots \ldots \ldots \ldots \ldots \ldots \ldots \ldots \ldots \ldots \ldots \ldots \ldots \ldots \\
& -\lambda_{m}\left[\left(n_{m}-p_{m m}^{1}\right) S_{1}\left(\hat{\tau}_{s}\right)+\left(n_{m}-p_{m m}^{2}\right) S_{2}\left(\hat{\tau}_{s}\right)+\right. \\
& \left.+\cdots+\left(n_{m}-p_{m m}^{m}\right) S_{m}\left(\hat{\tau}_{s}\right)\right] \\
& =-\left[\lambda_{m} n_{m}-\lambda_{1} p_{m 1}^{1}-\lambda_{2} p_{m 2}^{1}-\cdots-\lambda_{m} p_{m m}^{1}\right] S\left(\hat{\tau}_{s}\right)- \\
& -\left[\lambda_{m} n_{m}-\lambda_{1} p_{m 1}^{2}-\lambda_{2} p_{m z}^{2}-\cdots-\lambda_{m} p_{m m}^{2}\right] S_{2}\left(\hat{\tau}_{s}\right)- \\
& -\left[\lambda_{m} n_{m}-\lambda_{1} p_{m 1}^{m}-\lambda_{2} p_{m z}^{m}-\cdots-\lambda_{m} p_{m m}^{m}\right]_{m}\left(\hat{\tau}_{s}\right) \\
& \sum_{i=1}^{m} \lambda_{i} S_{m} S_{i}\left(\hat{\tau}_{s}\right)=-\left[\lambda_{m} n_{m}-\sum_{l=1}^{m} \lambda_{l} p_{m l}^{1}\right] S_{1}\left(\hat{\tau}_{s}\right)- \\
& -\left[\lambda_{m} n_{m}-\sum_{l=1}^{m} \lambda_{l} p_{m l}^{2}\right] S_{2}\left(\hat{\tau}_{s}\right)-\ldots- \\
& -\left[\lambda_{m} n_{m \overline{1}} \sum_{l=1}^{m} \lambda_{l} p_{m l}^{m}\right] S_{m}\left(\hat{\tau}_{g}\right) \text {. }
\end{aligned}
$$

Substituindo-se a expressao de (a.33) em (a.32), obtem-se a seguinte equacão: 


$$
\begin{aligned}
k S_{m}\left(Q_{s}\right)=u & {\left[\lambda_{m} n_{m}-\sum_{l=1}^{m} \lambda_{l} p_{m l}^{1}\right] S_{1}\left(\hat{\tau}_{s}\right)+} \\
& +u\left[\lambda_{m} n_{m}-\sum_{l=1}^{m} \lambda_{l} p_{m l}^{2}\right] S_{2}\left(\hat{\tau}_{s}\right)+\ldots+ \\
& +u\left[r(k-1)+\lambda_{m} n_{m}-\sum_{l=1}^{m} \lambda_{l} p_{m l}^{m}\right] S_{m}\left(\hat{\tau}_{s}\right) .
\end{aligned}
$$

As expressaes obtidas em (a.31) e (a.34) podem ser escritas numa forma geral, como:

$$
S_{i}\left(Q_{B}\right)=a_{i 1} S_{1}\left(\hat{\tau}_{B}\right)+a_{i 2} S_{2}\left(\hat{\tau}_{\theta}\right)+\ldots+a_{i m} S_{m}\left(\hat{\tau}_{B}\right)
$$

onde:

$$
a_{i j}=\left\{\begin{array}{c}
\frac{u r(k-1)}{k}+\frac{u}{k}\left(\lambda_{i} n_{i}-\sum_{l=1}^{m} \lambda_{l} p_{i l}^{j}\right), i=j \\
\frac{u}{k}\left(\lambda_{i} n_{i}-\sum_{l=1}^{m} \lambda_{l} p_{i l}^{j}\right), i \neq j
\end{array} \quad i, j=1,2, \ldots, m\right.
$$

De (a.35), forma-se o sequinte sistema de equaçzés

$$
\left[\begin{array}{cccc}
a_{11} & a_{12} & \cdots & a_{1 m} \\
a_{21} & a_{22} & \cdots & a_{2 m} \\
\cdots & \cdots & \cdots & \cdots \\
a_{m 1} & a_{m 2} & \cdots & a_{m m}
\end{array}\right]\left[\begin{array}{c}
S_{1}\left(\hat{\tau}_{s}\right) \\
S_{z}\left(\hat{\tau}_{B}\right) \\
\vdots \\
S_{m}\left(\hat{\tau}_{B}\right)
\end{array}\right]=\left[\begin{array}{c}
S_{1}\left(Q_{s}\right) \\
S_{2}\left(Q_{B}\right) \\
\cdots \\
S_{m}\left(Q_{s}\right)
\end{array}\right]
$$

Como $A$ é uma matriz formada pelos elementos $\left\{a_{i j}\right\}$, com inversa $A^{-1}$, de elementos $f_{i j}(i, j=1,2, \ldots, m)$, de acordo com CHAKRABARTI ( 1962 ), tem-se que:

$$
\left[\begin{array}{c}
S_{1}\left(\hat{\tau}_{s}\right) \\
S_{2}\left(\hat{\tau}_{s}\right) \\
\cdots \\
S_{m}\left(\hat{\tau}_{\theta}\right)
\end{array}\right]=\left[\begin{array}{cccc}
f_{11} & f_{12} & \cdots & f_{1 m} \\
f_{21} & f_{22} & \cdots & f_{2 m} \\
\cdots & \cdots & \cdots & \cdots \\
f_{m 1} & f_{m 2} & \cdots & f_{m m}
\end{array}\right]\left[\begin{array}{c}
S_{1}\left(Q_{s}\right) \\
S_{2}\left(Q_{s}\right) \\
\cdots \\
S_{m}\left(Q_{s}\right)
\end{array}\right]
$$

ou simplesmente,

$$
S_{i}\left(\hat{\tau}_{g}\right)=f_{i 1} S_{1}\left(Q_{g}\right)+f_{i z} S_{2}\left(Q_{s}\right)+\ldots+f_{i m} S_{m}\left(Q_{8}\right)(\Omega, 3 B)
$$


Substituindo-se a equaça de (a.37) na expressão encontrada em (a.24), tem-se que:

$$
\operatorname{ur}(k-1) \hat{\tau}_{8}=\left[\begin{array}{l}
k Q_{Q}+u \sum_{i=1}^{m} \lambda_{i} f_{i 1} S_{1}\left(Q_{8}\right)+u \sum_{i=1}^{m} \lambda_{i} f_{i 2} S_{2}\left(Q_{8}\right)+\ldots \\
\cdots+u \sum_{i=1}^{m} \lambda_{i} f_{i m} S_{m}\left(Q_{8}\right)
\end{array}\right.
$$

ou ainda,

$$
\begin{aligned}
& \operatorname{ur}(k-1) \hat{\tau}_{8}=k Q_{s}+u \sum_{j=1}^{m} s_{j}\left(Q_{8}\right) \sum_{i=1}^{m} \lambda_{i} f_{i j} \quad(a .39) \\
& \text { Assim, de (a.39) obtém-se que a solução de } \tau_{s} e: \\
& \hat{\tau}_{s}=\frac{1}{r(k-1)}\left[\frac{k}{u} Q_{B}+\sum_{j=1}^{m} s_{j}\left(Q_{8}\right) \sum_{i=1}^{m} \lambda_{i} f_{i j}\right] \quad \text { (a.40) }
\end{aligned}
$$

sendo a expressão que permite obter os efeitos ajustados de tratamentos primários para um $\mathrm{PBIB}(\mathrm{m})$.

Pode-se fazer, a partir de (a.39), uma generalizaçăo para a forma matricial $\hat{\tau}=M^{-1} Q$, sendo os elementos da matriz $M^{-1}=\left\{m_{i i}\right\}$ iguais a:

$$
m_{i i}= \begin{cases}k / u r(k-1) & \text {, se } i=i \\ \sum_{l=1}^{m} \lambda_{l} f_{l i} / r(k-1) & \text {, se } i \neq i\end{cases}
$$

\subsubsection{Estimaço dos efeitos de tratamentos secundários}

Reportando-se ao sistema de equaçoes normais, e resolvendo-se (a.12), tem-se que as soluças de minimos quadrados para os efeitos dos tratamentos secundários são determinadas a partir de:

$$
\hat{U \tau}^{*}=T^{*}-\operatorname{vr}{ }_{u_{1}} \hat{H}
$$

Sendo U uma matriz diagonal de posto completo, $r[U]=(u)$, então pré-multiplicando-se (a.42) por $U^{-1}$, obtém-se o vetor dos efeitos de tratamentos secundários:

$$
\hat{\tau}^{*}=U^{-1} T^{*}-{ }_{u} E_{1} \hat{\mu}
$$

Portanto,

$$
\hat{\mathrm{t}}_{\imath}^{*}=\frac{1}{\mathrm{vr}} \mathrm{T}_{\imath}^{*}-\hat{\mu}
$$

que e uma soluçao semelhante aquelas obtioas para iratamen- 
44.

tos secundários nos experimentos instalados em delineamento em blocos casualizados com parcelas subdivididas, e idêntica àquela encontrada por IBMMA (1981), para os experimentos em BIB .

\subsubsection{Estimaça dos efeitos de interaça}

Para a determinacão das soluços dos efeitos estimados das interaçzes ou pares de $\left(t t^{*}\right)_{i l}=\delta_{i l}$, substituem-se em (a.13) as expresszes de $\hat{\beta}=\mathrm{K}^{-1} B-K^{-1} N \cdot \hat{\tau}$ ${ }_{b} E_{1} \hat{\mu}$ obtida de (a.10), de $\hat{\tau}^{*}$ encontrada em (a.44), e

$$
\hat{\tau}=R^{-1} T-R^{-1} N \hat{\beta}-{ }_{V} E_{1} \hat{\mu}
$$

originada de (a.11).

Fazendo as substituiçres, tem-se que:

$$
\begin{aligned}
f_{v r} & E_{1} \hat{\mu}+S^{\prime}\left(R^{-1} T-R^{-1} N \hat{\beta}-v_{1} \hat{\mu}\right)+V\left(K^{-1} B-K^{-1} N \cdot \hat{\tau}-{ }_{b} E_{1} \hat{\mu}\right)+ \\
& +H^{\prime}\left(U^{-1} T^{*}-\mathrm{u}_{1} \hat{\mu}\right)+L \hat{\delta}=D
\end{aligned}
$$

desenvolvendo adequadamente, tem-se que:

$$
\begin{aligned}
& \mathrm{r}_{\mathrm{vr}} \mathrm{E}_{\mathbf{1}} \hat{\mu}+\mathrm{S}^{\prime} \mathrm{R}^{-1} \mathrm{~T}-\mathrm{S}^{\prime} \mathrm{R}^{-1} \mathrm{~N} \hat{\beta}-\mathrm{S}_{\mathrm{v}} \mathrm{E}_{1} \hat{\mu}+\mathrm{V}^{\prime} \mathrm{K}^{-1} \mathrm{~B}-\mathrm{V}^{\prime} \mathrm{K}^{-1} \mathrm{~N} \cdot \hat{\tau}- \\
& -V^{\prime} E_{1} \hat{\mu}+H^{\prime} U-T-H_{u} E_{1} \hat{\mu}+L \hat{\delta}=D \\
& \text { Substituindo a expressão de } \hat{\beta} \text {, obtém-se: } \\
& r_{V r} E_{1} \hat{\mu}+S^{\prime} R^{-1} T-S^{\prime} R^{-1} N K^{-1} B+S^{\prime} R^{-1} N K^{-1} N \cdot \hat{\tau}+ \\
& +S^{\prime} R^{-1} N_{b} E_{1} \hat{\mu}-S^{\prime} V_{1} \hat{\mu}+V^{\prime} K^{-1} B-V \cdot K^{-1} N \cdot \hat{\tau}-V^{\prime}{ }_{b} E_{1} \hat{\mu}+ \\
& +H^{\prime} U^{-1} T^{*}-H_{u}^{\prime} E_{1} \hat{\mu}+L \hat{\delta}=D \\
& \text { Posto que: } V^{\prime}=S^{\prime} R^{-1} N \text { e } \\
& S^{\cdot} \mathrm{E}_{1}=V^{\prime} \mathrm{E}_{1}=H^{\cdot} \mathrm{E}_{1}=r_{v u_{1}} E_{1}
\end{aligned}
$$

simplificando, fica:

$$
S^{\prime} R^{-1} T+H^{\prime} U^{-1} T^{*}-v_{r} E_{1} \hat{\mu}+L \hat{\mathcal{E}}=D
$$

então

$$
L \hat{\delta}=D-S^{\prime} R^{-1} T-H^{\prime} U^{-1} T^{*}+r_{v u} E_{1} \hat{\mu}
$$

Sendo L uma matriz não singular, de posto completo, $r[L]=v u$, a solução de mínimos quadrados para os efeitos da interação é dada por: 


$$
\hat{\mathcal{E}}=\mathrm{L}^{-1} \mathrm{D}-\mathrm{L}^{-1} \mathrm{~S} \cdot \mathrm{R}^{-1} \mathrm{~T}-\mathrm{L}^{-1} \mathrm{H} \cdot \mathrm{U}^{-1} \mathrm{~T}^{*}+{ }_{\mathrm{vu}} \mathrm{E}_{1} \hat{\mu} \quad(\mathrm{a} .46)
$$

onde,

$$
\begin{aligned}
& \text { vut }_{1} \in \text { um vetor de uns; } \\
& \mathrm{L}^{-1} \mathrm{D}=\frac{1}{\mathrm{r}}\left[\begin{array}{c}
\mathrm{TT}_{11}^{*} \\
\mathrm{TT}^{*} \\
\mathrm{TT}_{21}^{*} \\
\mathrm{TT}_{2 u}^{*} \\
\cdots_{v u}^{*} \\
\mathrm{TT}_{v 1}^{*} \\
\mathrm{TT}_{v u}^{*}
\end{array}\right]_{1}^{*}=\left\{\bar{y}_{\mathrm{i}, \iota_{\imath}}\right\} \\
& \epsilon \text { a media da combinação do } \\
& \text { i-esimo tratamento principal } \\
& \text { com } 0 \text { 1-esino tratamento } \\
& \text { secundario (sem ajuste para } \\
& \text { efeito de blocos) }
\end{aligned}
$$

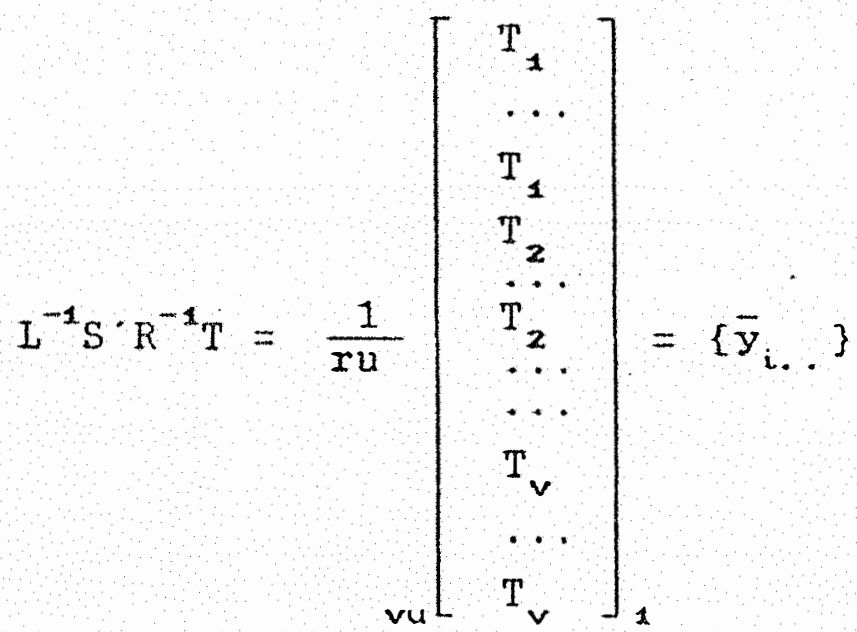

$$
\begin{aligned}
& \text { e a media do i-esimo } \\
& \text { tratamento principal (sem } \\
& \text { - ajuste para o efeito de } \\
& \text { blocos) }
\end{aligned}
$$


46.

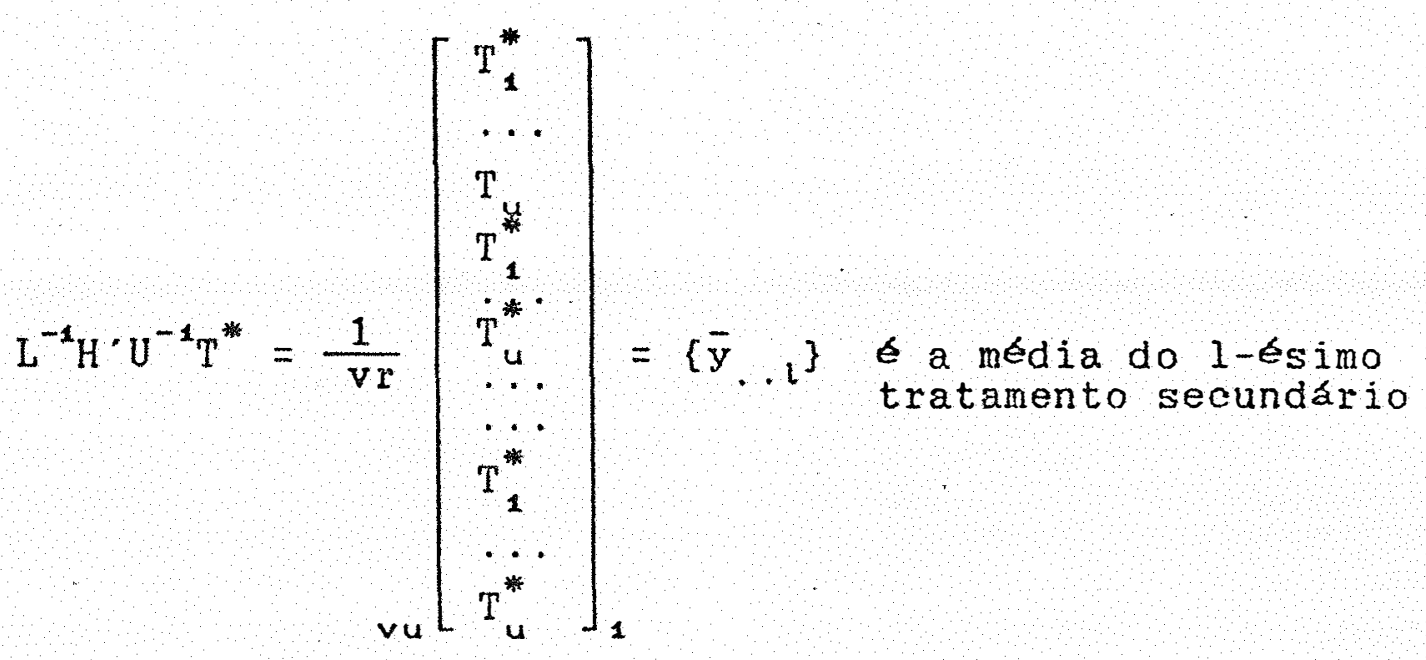

Assim, uma solução para o efeito estimado de um elemento qualquer da interaça, $e$ dada por:

$$
\hat{\delta}_{i l}=\bar{y}_{i, l}-\bar{y}_{i . .}-\bar{y}_{\ldots, l}+\bar{y}_{\ldots}
$$

que $\sigma$ um resultado identico aqueles obtidos em ensaios con parcelas subdivididas, envolvendo delineamentos eli blocos casualizados. Assim, verifica-se que os efeitos de interaça não são ajustados para blocos.

\subsubsection{PBIB com duas classes de associados}

Os delineamentos em bloco incompletos parcialmente balanceados com duas classes de associados, ou simplesmente $\operatorname{PBIB(2)}$, são os delinementos de particular importância, pelo fato de serem os mais utilizados na prática, por isso merecem uma maior atenço.

$$
\text { Para } m=2 \text {, de (a.39) tem-se que: }
$$

$$
\begin{aligned}
u r(k-1) \hat{\tau}_{s}=\left[k Q_{Q}+\right. & u\left(\lambda_{1} f_{11}+\lambda_{2} f_{21}\right) S_{1}\left(Q_{8}\right)+ \\
& \left.+u\left(\lambda_{1} f_{12}+\lambda_{2} f_{22}\right) S_{2}\left(Q_{B}\right)\right]
\end{aligned}
$$

Para estudar os elementos $f_{i j} \quad(i, j=1,2)$, considera-se a matriz $A$, como sendo:

$$
A=\left[\begin{array}{ll}
a_{11} & a_{12} \\
a_{21} & a_{22}
\end{array}\right]
$$

ande: 
(i)

$$
\begin{aligned}
a_{11} & =\frac{u}{k}\left[r(k-1)+\lambda_{1} n_{1}-\lambda_{1} p_{11}^{1}-\lambda_{2} p_{12}^{1}\right] \\
& =\frac{u}{k}\left[r(k-1)+\lambda_{1}\left(n_{1}-p_{11}^{1}\right)-\lambda_{2} p_{12}^{1}\right]
\end{aligned}
$$

Mas, de $(a .28)$ tem-se que $p_{11}^{1}+p_{12}^{1}=n_{1}-1$.

então

$$
\begin{aligned}
a_{11} & =\frac{u}{k}\left[r(k-1)+\lambda_{1}\left(1+p_{12}^{1}\right)-\lambda_{2} p_{12}^{1}\right] \\
& =\frac{u}{k}\left[r(k-1)+\left(\lambda_{1}-\lambda_{2}\right) p_{12}^{1}+\lambda_{1}\right]
\end{aligned}
$$

(ii) $\quad a_{12}=\frac{u}{k}\left[\lambda_{1} n_{1}-\lambda_{1} p_{11}^{2}-\lambda_{2} p_{12}^{2}\right]$

$$
\text { Mas, de (a.28) tem-se que } p_{11}^{2}+p_{12}^{2}=n_{1} \text {, }
$$

entao

$$
\begin{aligned}
a_{12} & =\frac{u}{k}\left[\lambda_{1} n_{1}-\lambda_{1}\left(n_{1}-p_{12}^{2}\right)-\lambda_{2} p_{12}^{2}\right] \\
& =\frac{u}{k}\left(\lambda_{1}-\lambda_{2}\right) p_{12}^{2}
\end{aligned}
$$

(iii) $a_{21}=\frac{u}{k}\left[\lambda_{2} n_{2}-\lambda_{1} p_{21}^{1}-\lambda_{2} p_{22}^{1}\right]$

então,

$$
\text { Mas, de (a.28) tem-se que } p_{21}^{1}+p_{22}^{1}=n_{2} \text {, }
$$

$$
\begin{aligned}
a_{21} & =\frac{u}{k}\left[\lambda_{2} n_{2}-\lambda_{1} p_{21}^{1}-\lambda_{2}\left(n_{2}-p_{21}^{1}\right)\right] \\
& =\frac{u}{k}\left[\left(\lambda_{2}-\lambda_{1}\right) p_{21}^{1}\right]
\end{aligned}
$$

(iv)

$$
\begin{aligned}
a_{22}= & \frac{u}{k}\left[r(k-1)+\lambda_{2} n_{2}-\lambda_{1} p_{21}^{2}-\lambda_{2} p_{22}^{2}\right] \\
& \text { Mas, de (a.28) tem-se que } p_{21}^{2}+p_{22}^{2}=n_{2}-1,
\end{aligned}
$$

entzo,

$$
\begin{aligned}
& a_{22}=\frac{u}{k}\left[r(k-1)+n_{2} \lambda_{2}-\lambda_{1} p_{21}^{2}-\lambda_{2}\left(n_{2}-1-p_{21}^{2}\right)\right] \\
& =\frac{u}{k}\left[r(k-1)+\left(\lambda_{2}-\lambda_{1}\right) p_{21}^{2}+\lambda_{2}\right] \\
& \text { Assim, a matriz } A^{-1} \text { com os elementos } f_{i j} \Leftrightarrow . \\
& A^{-1}=\left[\begin{array}{ll}
f_{11} & f_{12} \\
f_{21} & f_{22}
\end{array}\right]=\frac{1}{\Delta}\left[\begin{array}{cc}
a_{22} & -a_{12} \\
-a_{21} & a_{11}
\end{array}\right]
\end{aligned}
$$

onde, $\Delta=a_{11} a_{22}-a_{12} a_{21}$.

Então,

$$
\begin{aligned}
& f_{11}=a_{22} / \Delta, f_{12}=-a_{12} / \Delta \\
& f_{21}=-a_{21} / \Delta, f_{22}=a_{11} / \Delta
\end{aligned}
$$

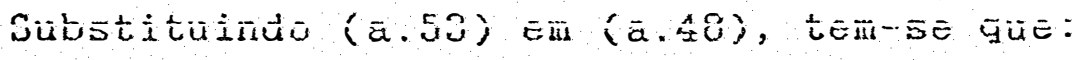




$$
\begin{aligned}
& \operatorname{ur}(k-1) \hat{\tau}_{\theta}=k Q_{\theta}+u\left(\lambda_{1} a_{22} / \Delta-\lambda_{2} a_{21} / \Delta\right) S_{1}\left(Q_{\theta}\right)+ \\
& +u\left(\lambda_{2} a_{11} / \Delta-\lambda_{1} a_{12} / \Delta\right) S_{2}\left(Q_{8}\right) \\
& =k Q_{8}+\frac{u}{\Delta}\left[\left(\lambda_{1} a_{22}-\lambda_{2} a_{21}\right) S_{1}\left(Q_{\theta}\right)+\left(\lambda_{2} a_{11}-\lambda_{1} a_{12}\right) S_{2}\left(Q_{\theta}\right)\right] \\
& =k Q_{8}+\frac{u}{\Delta}\left[A_{1} S_{1}\left(Q_{8}\right)+A_{2} S_{2}\left(Q_{8}\right)\right]
\end{aligned}
$$

como $A_{1}=\left(\lambda_{1} a_{22}-\lambda_{2} a_{21}\right)$ e $A_{2}=\left(\lambda_{2} a_{11}-\lambda_{1} a_{12}\right)$

então, a soluça de $\tau_{a}$ é:

$$
\hat{\tau}_{\theta}=\frac{1}{r(k-1) \Delta}\left[\frac{k \Delta}{u} Q_{s}+A_{1} S_{1}\left(Q_{s}\right)+A_{2} S_{2}\left(Q_{\theta}\right)\right]
$$

sendo a expressão que permite obter os efeitos ajustados de tratamentos primários para um $\operatorname{PBIB}(2)$.

Para maior facilidade de cálculos, quando ocorrer $n_{1}<n_{2}$, e preferivel eliminar $S_{2}\left(Q_{8}\right)$, de $(a .54)$. Assim, considerando-se a propriedade, como $Q_{s}+S_{1}\left(Q_{8}\right)+$ $S_{2}\left(Q_{s}\right)=0$, tem-se que:

$$
S_{2}\left(Q_{8}\right)=-Q_{8}-S_{1}\left(Q_{8}\right)
$$

Substituindo-se $(a .55)$ en $(a .54)$, obtém-se:

$$
\hat{\tau}_{s}=\frac{k}{u r(k-1)} Q_{s}+\frac{1}{r(k-1) \Delta}\left\{A_{1} S_{1}\left(Q_{s}\right)-A_{2}\left[Q_{s}+S_{1}\left(Q_{s}\right)\right]\right\}
$$

segue-se que:

$$
\begin{array}{r}
\left.\hat{\tau}_{s}=\frac{1}{u r(k-1) \Delta}\left[\left(k \Delta-u A_{2}\right) Q_{8}+u\left(A_{1}-A_{2}\right) S_{1}\left(Q_{s}\right)\right] \text { (a. } 56\right) \\
\text { Uma generalizaça para a forma matricial }
\end{array}
$$
$\hat{\tau}=M^{-1} Q$, sendo os elementos da matriz $M^{-1}=\left\{m_{i i}\right\}$ iguais $a$ :

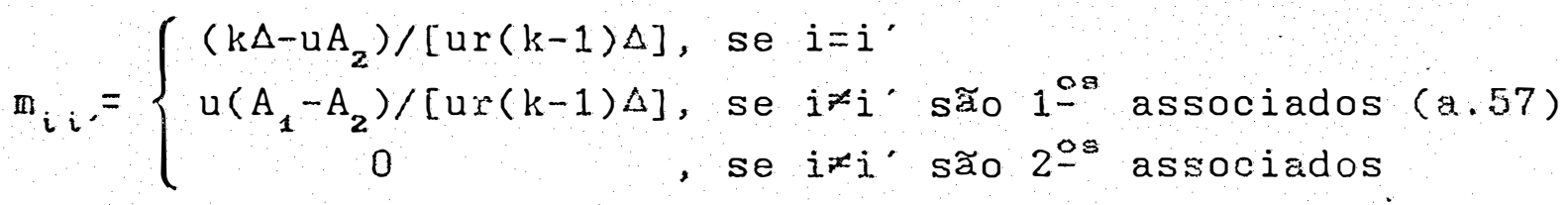

Quando $n_{1}>n_{2}$, é preferível eliminar $S_{1}\left(Q_{8}\right)$ de (a.54). Assim, considerando-se a propriedade, como $Q_{8}+$ $S_{1}\left(Q_{s}\right)+S_{2}\left(Q_{s}\right)=0$

tem-se que :

$$
S_{1}\left(Q_{\varepsilon}\right)=-Q_{8}-S_{2}\left(Q_{8}\right)
$$

Substituindo-se (a.58) em (a.54), obtem-se: 


$$
\hat{r}_{s}=\frac{k}{\operatorname{ur}(k-1)} Q_{s}+\frac{1}{r(k-1) \Delta}\left\{-A_{1}\left[Q_{s}+S_{2}\left(Q_{s}\right)\right]+A_{2} S_{2}\left(Q_{s}\right)\right\}
$$

segue-se que:

$$
\hat{\tau}_{s}=\frac{1}{\operatorname{ur}(k-1) \Delta}\left[\left(k \Delta-u A_{1}\right) Q_{s}+u\left(A_{2}-A_{1}\right) S_{2}\left(Q_{s}\right)\right]
$$

Uma generalizaço para a forma matricial $\hat{\tau}=M^{-1} Q$, sendo os elementos da matriz $M^{-1}=\left\{m_{i i^{\prime}}\right\}$ iguais a:

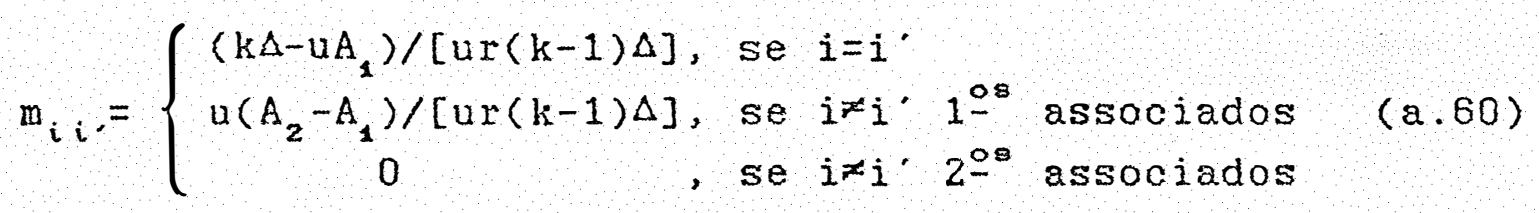

Para o exemplo ilustrativo de (3.1), tem-se:

$$
\begin{aligned}
& \lambda_{1}=1, \lambda_{2}=0, i=s=2, v=4, b=4, r=2, k=2, n_{1}=2, n_{2}=1 \\
& a_{11}=6, a_{12}=0, a_{21}=-3 / 2, a_{2 z}=3, A_{1}=3, A_{2}=0, \Delta=18 \\
& \hat{\tau}_{s}=\frac{1}{3.2 \cdot 1 \cdot 18}\left[(2.18-3.3) Q_{8}+3(0-3) s_{2}\left(Q_{s}\right)\right] \\
& \hat{\tau}_{s}=\frac{1}{12}\left[3 Q_{s}-s_{2}\left(Q_{s}\right)\right]
\end{aligned}
$$

3.4.3. Caso espectal: Experímentos em blocos incompletos balanceados

As soluçes para os efeitos de tratamentos principais (ajustados) podem ser obtidas, nesse caso, a partir de um delineamento PBIB, considerando-se $\lambda_{1}=\lambda_{2}=\ldots$ $=\lambda_{m}=\lambda$. Por exemplo, tomando-se um PBIB con duas olasses de associados, tem-se que:

$$
\begin{aligned}
& a_{11}=\frac{u}{k}[r(k-1)+\lambda]=a_{22} ; a_{12}=a_{21}=0 ; \\
& A_{1}=A_{2}=\frac{u}{k}[r(k-1)+\lambda] \lambda ; \quad \Delta=\frac{u^{2}}{k^{2}}[r(k-1)+\lambda]^{2} \\
& \quad \text { Portanto, } \\
& \hat{\tau}_{a}=\frac{1}{u r(k-1) \Delta}\left[k \Delta-u A_{z}\right]=\frac{1}{u r(k-1)}\left[k-\frac{u}{\Delta} A_{z}\right] Q_{s}=
\end{aligned}
$$




$$
\begin{aligned}
\hat{r}_{s} & =\frac{1}{u r(k-1)}\left[k-\frac{u \frac{u}{k}[r(k-1)+\lambda] \lambda}{\frac{u^{2}}{k^{2}}[r(k-1)+\lambda]^{2}} Q_{s}=\right. \\
& =\frac{1}{u r(k-1)}\left[k-\frac{\lambda k}{r(k-1)+\lambda}\right] Q_{s}= \\
& =\frac{1}{u r(k-1)}\left[\frac{k[r(k-1)+\lambda]-\lambda k}{r(k-1)+\lambda}\right] Q_{s}= \\
& =\frac{1}{u r(k-1)} \frac{r k(k-1)}{r(k-1)+\lambda} Q_{s}
\end{aligned}
$$

mas, lembrando-se que em $B I B$, tem-se que $\lambda(v-1)=r(k-1)$, segue-se que:

$$
\hat{\tau}_{Q}=\frac{k}{\lambda v u} Q_{B}
$$

que $\Leftrightarrow$ a expressão da soluçäo encontrada por IEMMA (1981).

\subsubsection{Experimentos em reticulados quadrados}

Segundo PIMENTEL GOMES (1954), considerar os reticulados quadrados como um caso particular dos delineamentos em PBIB, parece ser, um procedimento pouco aconselhável, pois a análise desses experimentos é mais complexa do que aquelas dos experimentos em reticulados quadrados; por este modo, acha-se por bem, acrescentar algumas consideraçes sobre este tópico.

Do SENR de (a.19), tem-se que

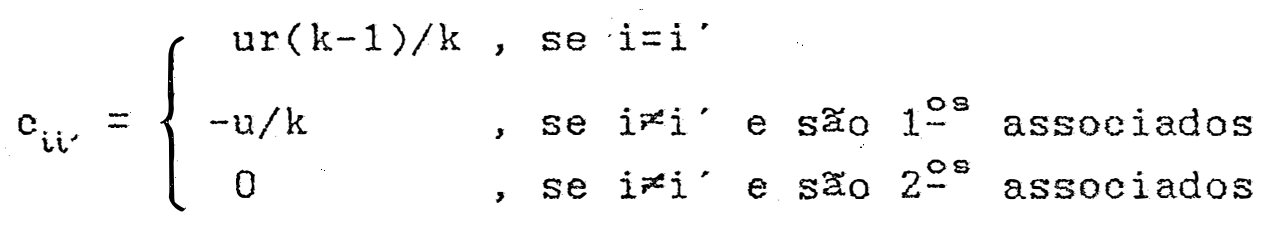

As equaços normais para as dois primeiros tratamentos principais do primeiro bloco ou relativa aos parametros $t_{1}$ e $t_{2}$, considerando-se o exemplo ilustrativo de (3.1), são:

$$
\begin{array}{r}
u r\left(1-\frac{1}{k}\right) \hat{t}_{1} \quad-\frac{u}{k} \hat{t}_{2}-\frac{u}{k} \hat{t}_{9}-\frac{a}{k} \hat{t}_{4}=a_{1} \\
-\frac{u}{k} \hat{t}_{1}+u r\left(1-\frac{1}{k}\right) \hat{t}_{2}-0 \hat{t}_{3}-\frac{u}{k} \hat{t}_{4}=a_{2}
\end{array}
$$


Somando e subtaindo $\frac{\mathrm{ur}}{\mathrm{k}} \hat{\mathrm{t}}_{\mathrm{i}} \quad(i=1,2, \ldots, \mathrm{k})$ as equaçæes anteriores, tem-se $u r\left(1-\frac{1}{k}\right) \hat{t}_{1}-\frac{u}{k}\left(\hat{t}_{1}+\hat{t}_{2}\right)-\frac{u}{k}\left(\hat{t}_{1}+\hat{t}_{9}\right)+\frac{u r}{k} \hat{t}_{1}=Q_{1}$ $u r\left(1-\frac{1}{k}\right) \hat{t}_{2}-\frac{u}{k}\left(\hat{t}_{1}+\hat{t}_{2}\right)-\frac{u}{k}\left(\hat{t}_{2}+\hat{t}_{1}\right)+\frac{u r}{k} \hat{t}_{2}=Q_{2}$ Definindo $S_{j}\left(t_{i}\right)$ como a soma dos efeitos de tratamentos principais que ocorrem juntos com o i-ésimo tratamento principal na j-ésima repetiçăo. Assim fica

$$
\begin{aligned}
& u r \hat{t}_{1}-\frac{u}{k} s_{1}\left(\hat{t}_{1}\right)-\frac{u}{k} s_{2}\left(\hat{t}_{1}\right)=Q_{1} \\
& u r \hat{t}_{2}-\frac{u}{k} s_{1}\left(\hat{t}_{2}\right)-\frac{u}{k} s_{2}\left(\hat{t}_{2}\right)=Q_{2}
\end{aligned}
$$

Somando as equaços (a.63) e(a.64) relativas ao primeiro bloco, e considerando que se verifica a igualdade $S_{1}\left(\hat{t}_{1}\right)=S_{1}\left(\hat{t}_{2}\right)=k S_{1}\left(\hat{t}_{1}\right)$ tem-se

$$
\operatorname{urS}_{1}\left(\hat{t}_{1}\right)-\frac{u}{k} k S_{1}\left(\hat{t}_{1}\right)-\frac{u}{k}\left[S_{2}\left(\hat{t}_{1}\right)+S_{2}\left(\hat{t}_{2}\right)\right]=S_{1}\left(Q_{1}\right) \quad(a .65)
$$

onde $S_{j}\left(Q_{i}\right)$ e a soma dos $Q$ s referentes aos tratamentos que ocorrem num mesmo bloco com o i-esimo tratamento principal na j-essima repetisão. Assim, tem-se

$$
s_{2}\left(\hat{t}_{1}\right)+s_{2}\left(\hat{t}_{2}\right)=\hat{t}_{1}+\hat{t}_{3}+\hat{t}_{2}+\hat{t}_{4}=\sum_{i=1}^{v} \hat{t}_{i}=0
$$

portanto,

$$
\begin{gathered}
u(r-1) S_{1}\left(\hat{t}_{1}\right)=S_{1}\left(Q_{1}\right) \\
S_{j}\left(\hat{t}_{i}\right)=S_{j}\left(Q_{i}\right) / u(r-1)(i=1, \ldots, v ; j=1, \ldots, r)
\end{gathered}
$$

Substituindo a express̃̃o (a.68) em (a.63),

tem-se

$$
\begin{array}{r}
u \hat{t}_{1}-\frac{u}{k}\left[\frac{1}{u(r-1)} S_{1}\left(Q_{1}\right)+\frac{1}{u(r-1)} S_{2}\left(Q_{1}\right)\right]=Q_{1} \\
\hat{t}_{1}=\frac{1}{u r} Q_{1}+\frac{1}{u k r(x-1)}\left[S_{1}\left(Q_{1}\right)+S_{2}\left(Q_{1}\right)\right]
\end{array}
$$

Generalizando, tem-se que os efeitos de tratamentos principais são obtidos por:

$$
\hat{t}_{i}=\frac{1}{u k r(r-1)}\left[k(r-1) Q_{i}+S_{1}\left(Q_{i}\right)+S_{2}\left(Q_{i}\right)+\ldots+S_{r}\left(Q_{i}\right)\right]
$$

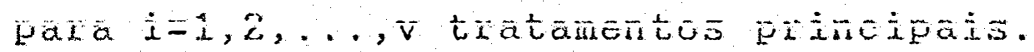


52 .

Pode-se obter uma generalização para a solução $\hat{\tau}=M^{-1} Q$, atraves dos coeficientes dos $Q^{\prime} s$. Os elementos $\left\{m_{i i} \cdot\right\}$, da matriz $M^{-1}$, são

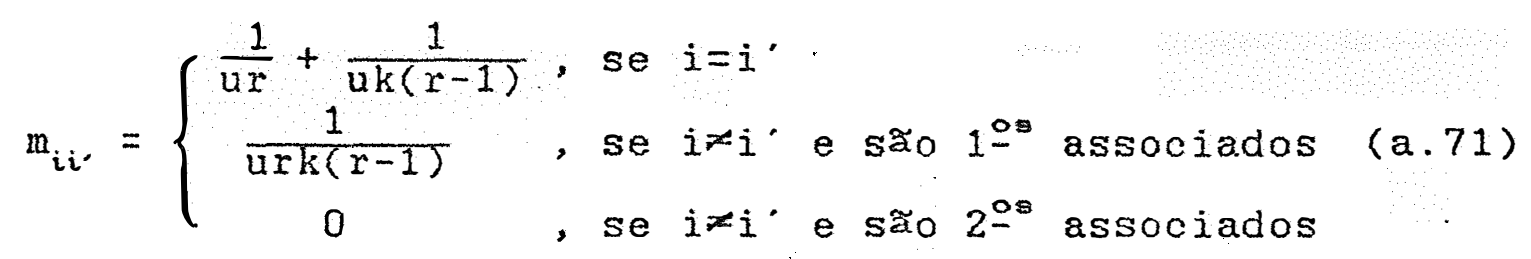

Para o exemplo ilustrativo de (3.1), tem-se:

$$
\begin{aligned}
& m_{i i}=\left\{\begin{array}{l}
\frac{1}{3(2)}+\frac{1}{3(2)(1)}=\frac{4}{12}, \text { se } i=i \\
\frac{1}{3(2) 2(1)}=\frac{1}{12}, \text { se } i=i \cdot \text { e são primeiros associados } \\
0, \text { se } i<i \cdot \text { e são segundos associados }
\end{array}\right. \\
& \hat{t}_{s}=\frac{1}{3 \cdot 2} Q_{i}+\frac{1}{3 \cdot 2 \cdot 2 \cdot 1}\left[S_{1}\left(Q_{s}\right)+S_{2}\left(Q_{s}\right)\right]= \\
& =\frac{1}{6} Q_{s}+\frac{1}{12}\left[S_{1}\left(Q_{s}\right)+S_{2}\left(Q_{s}\right)\right]
\end{aligned}
$$

\subsection{Matriz de variancias e covariancias}

Do modelo adotado em (a.1), as pressuposiços associadas aos erros entre subparcelas, consideradas independentes, e de uso tradicional nos experimentos planejados em parcelas subdivididas, de (a.4), obteve-se a seguinte matriz de variancias e covariâncias:

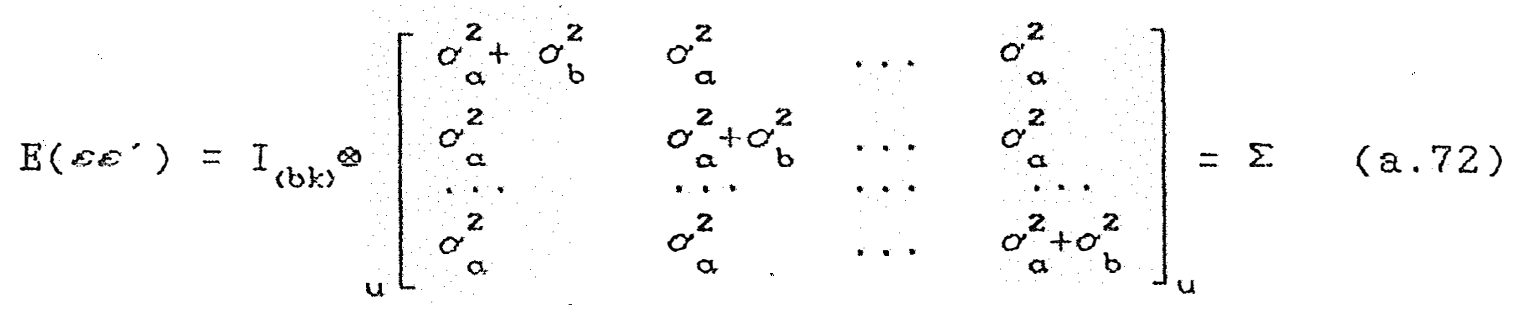

Assim, existem bk = vr submatrizes uniformes na posiça diagonal, e as demais submatrizes săo todas nulas; ou seja, existe uma matriz uniforme para cada uma das vr parcelas. Como a matriz de covariancias adotada e do tipo uniforme, que pressupoe variancias e covariancias constan-

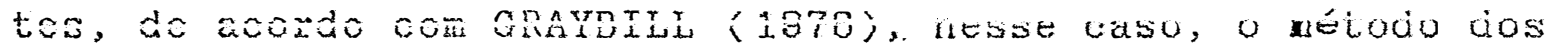


mínimos quadrados (ordinários) pode ser utilizado em lugar dos minimos quadrados generalizados.

Para experimentos em parcelas subdivididas, os testes de homogeneidade e uniformidade das matrizes de covariancias, entre os diferentes tratamentos secundários dentro de cada tratamento principal, são requisitos básicos para a adequação do modelo, de acordo com DANFORD et alii (1960), IEMMA (1981) e HILRS (1946).

Face a adequação do modelo proposto aos dados experimentais, e visto que năo é raro encontrar correlaçఠes entre os erros experimentais, se não são verificadas as hipóteses de homogeneidade e uniformidade, uma alternativa é - uso das técnicas de analise multivariada, de acordo com DANFORD et alii (1960), GILL (1978) e LEAL (1979).

\subsubsection{Teste de homogeneidade}

A suposiçăo de homogeneidade, de uma matriz de covariancias para todos os tratamentos principais, pode-se ser verificada utilizando-se do méto proposto por Box (1950), como uma extensão do teste multivariado de homogeneidade de variancias de Bartlett (1957), descrito em DANFORD et alii (1980) e PEARSON (1989).

A hipótese a ser verificada é

$H_{0}: \Sigma_{1}=\ldots=\Sigma_{v}=\Sigma$ vs $H_{\alpha}: \Sigma_{i} \neq \Sigma$ para algum $i=1, \ldots, \mathrm{v}$ onde $\Sigma_{i}$ é a matriz de covariancias do i-ésimo tratamento primário ou principal.

$$
\begin{aligned}
& \text { O critério do teste é } \\
& M=(r-1) v \ln \left|S_{u} \cdot\right|-(r-1) \sum_{i=1}^{v} \ln \left|S_{u \cdot i}\right|
\end{aligned}
$$

onde:

(i) $S_{u l}, \epsilon$, sob hipótese de nulidade, uma estimativa não viesada da matriz de covariancia $\Sigma$, associada com $v(r-1)$ graus de liberdade, composta por:

$$
s_{l l}^{2}=\frac{1}{v(r-1)} \sum_{i=1}^{v}\left[\sum_{j=1}^{r} y_{i j t} y_{i j l}-\frac{y_{i, l} y_{i . L}}{r}\right]
$$

correspondente a estimativa da matriz de covariancias entre 
54.

os 1 e l'-ésimos tratamentos secundários; e

(ii) $S_{l l i} \in$ a estimativa ño viesada de $\Sigma_{i}$, associada com r-1 graus de liberdade, sendo composta por:

$$
s_{l L i}^{2}=\frac{1}{(r-1)}\left[\sum_{j=1}^{r} y_{i j l} y_{i j l}-\frac{y_{i, l} y_{i, l}}{r}\right]
$$

correspondente a estimativa das matrizes de covariancias entre os 1 e l'-ésimos tratamentos secundarios para um dado i-esimo tratamento primário.

$$
\text { Assim, }\left(1-A_{1}\right) M, \quad \operatorname{com}
$$

$A_{1}=\left(2 u^{2}+3 u+1\right)(v+1) /[6 v(u+1)(r-1)]$, tem distribuiça aproximada de $x^{2}$ com $f_{1}=(v-1) u(u+1) / 2$ graus de liberdade.

Portanto, rejeita-se a hipótese de homogeneidade de variancias e covariancias se $\left(1-A_{1}\right) M \geq \chi_{\left(\alpha_{1} f_{1}\right.}^{2}$

\subsubsection{Teste de uniformidade}

A suposiç̃o de uniformidade de uma matriz de covariâncias pode ser verificada atraves do criterio da razão de verossimilhança de Neyman-Pearson, modificado por Box (1949).

$$
\begin{aligned}
& \text { A hipotese a ser testada } 6 \\
& H_{0}: \Sigma=\Sigma^{*} \text { vs } H_{a}: \Sigma \not \Sigma^{*}
\end{aligned}
$$

onde $\Sigma^{*} \leqslant$ uma matriz do tipo uniforme, ou seja

$$
\Sigma^{*}=\left[\begin{array}{cccc}
\sigma_{1} & \sigma_{2} & \cdots & \sigma_{2} \\
\sigma_{2} & \sigma_{1} & \cdots & \sigma_{2} \\
\sigma_{2} & \sigma_{2} & \cdots & \sigma_{1}
\end{array}\right]
$$

onde: $\sigma_{1}=\sigma_{a}^{2}+\sigma_{b}^{2}$ e $\sigma_{2}=\sigma_{a}^{2}$.

$$
0 \text { criterio da teste é }
$$

$$
N=-(v r-v) \text { In }[\operatorname{det}(S) / \operatorname{det}(\bar{S})]
$$

onde: $S=\left\{s_{11}^{2},\right\}$

$$
\begin{aligned}
& \bar{S}=U \Leftrightarrow \text { uma matriz uniforme composta por: } \\
& u_{u} \text { e a média das variāncias de } \mathrm{s}^{2}\left(1=1^{\prime}\right) \text {; } \\
& \left.\left.u_{u} \text {, é a média das covariâncias de } s_{l l}^{2} \text {, ( } 1 x^{\prime}\right]^{\prime}\right) \text {. } \\
& \text { Assim, }\left(1-A_{1}\right) N, \quad \operatorname{com} \\
& \hat{A}_{*}=u(u+1)^{2}(2 u-3) /\left[6(v r-v)(u-1)\left(u^{2}+u-4\right)\right], \text { teni distribujo }
\end{aligned}
$$


aproximada de $x^{2}$ com $f_{1}=\left(u^{2}+u-4\right) / 2$ graus de liberdade.

Portanto, rejeita-se a hipótese de uniformidade $\operatorname{se}\left(1-A_{1}\right) N \geq \chi_{\left(a, f_{1}\right)^{2}}$.

\subsection{Propriedades das solucoes}

3.6.1. Esperanqa matematica de $\hat{\tau}$

Considerando-se o modelo dado em $(a .1)$, e

usando o mesmo procedimento de PIMENTEL GOMES (1968), tem-se que:

$$
\hat{\tau}=M^{-1} Q=M^{-1} W X \cdot Y,
$$

onde, $Q=W X^{\prime} y e$ W ${ }^{\prime}=$ matriz de dimensao $(v \times n)$, tal que então,

$$
W=\left[_{v} \phi_{1}\left(-N K^{-1}\right)_{b} I_{v} v_{v}^{\phi} v_{v u}^{\phi}\right] \quad(a .78)
$$

$$
\begin{gathered}
E(\hat{r})={ }_{\text {Mas, }}\left(M^{-1} Q\right)=H^{-1} W X \cdot E(Y)=M^{-1} W X \cdot X \theta \\
\text { W }
\end{gathered}
$$

$W X^{\prime} X \theta=\left[-N K^{-1} X_{2}^{\prime}+X_{9}^{\prime}\right]\left[X_{1} \mu+X_{2} \beta+X_{3} \tau+X_{4} \tau^{*}+X_{55} \delta\right]$

fazendo-se as multiplicaçöes e aplicando-se as restricöes de (a.8), tem-se

$$
\begin{aligned}
& W X^{\prime} X \theta=\left(R-N K^{-1} N^{\prime}\right) \tau=C \tau \\
& \text { Assim, voltando-se em }(a .79) \text {, obtem-se que } \\
& E(\hat{\tau})=M^{-1} \mathrm{C} \tau
\end{aligned}
$$

mas, de PIMENTEL GOMES (1968) e OLIVEIRA (1985), tem-se que se $C=M+A$, onde $A \in$ uma matriz de restriços, tal que $A \tau=\varnothing$, então

$$
E(\hat{\tau})=M^{-1}(M+A) \tau=\tau+M^{-1} A \tau
$$

Logo,

$$
E(\hat{\tau})=\tau
$$

ou seja, $\hat{\tau}$ e um estimador năo viesado de $\tau$.

3.6.2. Matriz de disperszo para os tratamentos principais

A matriz de dispersão para os tratamentos principais, foi obtida considerando-se por definiça que:

$$
D(\hat{\tau})=E\left\{[\hat{\tau}-E(\hat{\tau})][\hat{\tau}-E(\hat{\tau})]^{\prime}\right\}
$$


Mas, de (a.77) e (a.80), tem-se que

$\hat{\tau}-\mathrm{E}(\hat{\tau})=\mathrm{M}^{-1} \mathrm{WX}(\mathrm{X} \theta+\varepsilon)-\mathrm{M}^{-1} \mathrm{C} \tau=\mathrm{M}^{-1} W X^{\prime} \varepsilon$

Desse modo, tem-se

$D(\hat{\tau})=E\left\{\left[M^{-1} W X^{\prime} \varepsilon\right]\left[M^{-1} W X^{\prime} \varepsilon\right]\right\}=M^{-1} W^{\prime} E\left(\varepsilon \varepsilon^{\prime}\right) X W^{\prime} M^{-1}$

$$
=M^{-1} W X \cdot \Sigma X W^{\prime} M^{-1}
$$

Efetuando-se o produto $W^{\prime} \Sigma$, obteve-se

$$
W X^{\prime} \Sigma=\left(\sigma_{b}^{2}+u \sigma_{a}^{2}\right) W X^{\prime}
$$

ent $\approx 0,(a .83)$, resulta em

$$
\begin{aligned}
& D(\hat{\tau})=\left(\sigma_{b}^{2}+u \sigma_{a}^{2}\right) M^{-1} W X X W^{\prime} M^{-1} \\
& \text { Mas, }
\end{aligned}
$$

$W^{\prime} X^{\prime}=\left(X_{3}^{\prime}-N K^{-1} X_{2}^{\prime}\right)\left(X_{9}-X_{2} K^{-1} N^{\prime}\right)$

pazendo-se as multiplicaçres e simplificando, obtem-se $W^{\prime} X^{\prime}=X_{3}^{\prime} X_{3}-X_{3}^{\prime} X_{2} K^{-1} N^{\prime}=R-N K^{-1} N^{\prime}=C$

\section{Desse modo,}

$$
D(\hat{\tau})=\left(\sigma_{b}^{2}+u \sigma_{a}^{2}\right) M^{-1} \mathrm{CM}^{-1}
$$

resultado semelhante ao obtido por IEMMA (1981), para a matriz de dispersão dos tratamentos principais em experimentos em blocos incompletos balanceados (BIB).

$$
\begin{aligned}
& \text { 3.6.3. Esperança matematica de } \hat{\tau}^{*} \\
& \text { De (a.43), tem-se que } \\
& r^{*}=U^{-1} T^{*}-U^{-1} U^{1} \mu=U^{-1} T^{*}-U^{-1} v_{r} E_{1} \frac{1}{v r u} X_{1} y \\
& =U^{-1} X_{4} y-E_{1}\left(X_{1} X_{1}\right)^{-1} X_{1} y \\
& =\left[U^{-1} X_{4}-u_{1}^{E}\left(X_{1}^{\prime} X_{1}\right)^{-1} X_{1}^{\prime}\right] y=P_{s} y \\
& E\left(\hat{\tau}^{*}\right)=E\left[P_{S}(X+\varepsilon)\right]=P_{S} X \theta
\end{aligned}
$$

Mas,

$P_{s} X \theta=\left[U^{-1} X_{4}^{\prime}-\mathrm{U}_{1}\left(X_{1}^{\prime} X_{1}\right)^{-1} X_{1}^{\prime}\right]\left(X_{1} \mu+X_{2} \beta+X_{9} \tau+X_{4} \tau^{*}+X_{5} \delta\right)$ Fazendo-se as multiplicaçres e aplicando-se as restriçðes de (a.8), tem-se:

$\log 0$,

$$
P_{S} X \theta=U^{-1} X_{4}^{\prime} X_{4} \tau^{*}=\tau^{*}
$$

$$
E\left(\hat{\tau}^{*}\right)=\tau^{*}
$$


ou seja, $\hat{\tau} \approx$ en estimador não viesado de $\tau^{*}$.

\subsubsection{Matriz de dispersa para os tratamentos se- cundarios}

Aplicando-se a definiçăo de matriz de dispersăo, tem-se que

$$
\begin{gathered}
D\left(\tau^{*}\right)=E\left\{\left[\tau^{*}-E\left(\tau^{*}\right)\right]\left[\tau^{*}-E\left(\tau^{*}\right)\right]^{\prime}\right\} \\
\operatorname{De}(a .86), \text { tem-se } \\
\hat{\tau}^{*}-E(\hat{\tau})=P_{S} \varepsilon
\end{gathered}
$$

3.ssim, $(2.88)$ fica

$$
D(\hat{\tau})=E\left[\left[P_{s^{*}} \varepsilon\right]\left[P_{s} \varepsilon\right]^{\prime}\right\}=P_{s} E\left(\varepsilon \varepsilon^{\prime}\right) P_{s}^{\prime}=P_{s} \Sigma P_{s}^{\prime}
$$

onde,

$$
\begin{aligned}
& P_{s}=E_{b k} \otimes \frac{1}{v u r}\left[\begin{array}{cccc}
u-1 & -1 & \cdots & -1 \\
-1 & u-1 & \cdots & -1 \\
\cdots & \cdots & \cdots & \cdots \\
-1 & -1 & \cdots & u-1
\end{array}\right]_{u} \\
& D\left(\hat{r}^{*}\right)=c_{b}^{2} P_{s} s_{s}, \text { mas } \\
& \text { Verifica-se que } P_{s} \Sigma=\sigma_{b}^{2} P_{s} \text {, e portanto, } \\
& P_{s}{ }^{\prime}=\left[U^{-1} X_{4}-{ }_{u} E_{1}\left(X_{1} X_{1}\right)^{-1} X_{1}\right]\left[U^{-1} X_{1}-u_{1}\left(X_{1} X_{1}\right)^{-1} X_{1}\right] \\
& =U^{-1} X_{4} X_{4} U^{-1}-2 U^{-1} X_{4} X_{1}\left(X X_{1}\right)^{-1} E_{u}+{ }_{u} E_{1}\left(X_{1} X_{1}\right)^{-1} E_{u}
\end{aligned}
$$

simplificando, obtem-se

$$
\begin{gathered}
P_{s} s^{P}=\frac{1}{v r} I_{(u)}-\frac{1}{v r u} E_{(u)} \\
\text { Desse modo, } \\
D\left(\hat{\tau}^{*}\right)=\frac{1}{v r u}\left[\begin{array}{cccc}
u-1 & -1 & \cdots & -1 \\
-1 & u-1 & \cdots & -1 \\
\cdots & \cdots & \cdots & \cdots \\
-1 & -1 & \cdots & u-1
\end{array}\right]_{u} \sigma_{b}^{2}
\end{gathered}
$$

3.6.5. Esperanga matematica da interaşa $\hat{\mathcal{S}}$

$$
\text { De }(2.46) \text {, tem-se que }
$$

$\hat{b}=L^{-1} D-L^{-1} S \cdot R^{-1} T-L^{-1} H \cdot U^{-1} T^{*}+L^{-1} r_{V U} E_{i} G / n$ 
58.

$$
\begin{aligned}
& =\left[L^{-1} X_{5}^{\prime}-L^{-1} S^{\prime} R^{-1} X_{9}^{\prime}-L^{-1} H^{\prime} U^{-1} X_{4}+v_{u} E_{1}\left(X_{1}^{\prime} X_{1}\right)^{-1} X_{1}^{\prime}\right] y \\
& =P_{1} y
\end{aligned}
$$

aplicando-se a esperanca matematica, fica

$$
\begin{aligned}
& E(\hat{\delta})=E\left(P_{x} y\right)=P_{I} E(y)=P_{I} E(X \theta+\varepsilon)=P_{I} X^{\theta} \\
& \text { Mas, } \\
& P_{I} X \theta=L^{-1} X_{5}\left(X_{1} \mu+X_{2} \beta+X_{9}{ }^{2}+X_{4}{ }^{*}+X_{5} \delta\right)- \\
& -L^{-1} S^{\prime} R^{-1} X_{3}\left(X_{1} \mu+X_{2} \beta+X_{3} \tau+X_{4} \tau+X_{5} \delta\right)- \\
& -\mathrm{L}^{-1} \mathrm{H} \cdot \mathrm{U}^{-1} \mathrm{X}_{4}\left(\mathrm{X}_{1} \mu+\mathrm{X}_{2} \beta+\mathrm{X}_{3} \tau+\mathrm{X}_{4} \tau+\mathrm{X}_{5} \delta\right)+ \\
& { }^{v_{u}} E_{1}\left(X_{1}^{\prime} X_{1}\right)^{-1} X_{1}^{\prime}\left(X_{1} \mu+X_{2} \beta+X_{3} \tau+X_{4} \tau+X_{5} \delta\right)
\end{aligned}
$$

Fazendo-se as multiplicaçes, e aplicando-se as restriços de $(a .8)$ e, usando algumas igualdades entre as matrizes de modo conveniente, verifica-se que:

$$
P_{I} X \theta=\delta
$$

Então, obtem-se que

$$
E(\hat{\delta})=\delta
$$

ou seja, $\hat{\delta} \varepsilon$ um estimador não viesado de $\delta$.

\subsubsection{Matriz de dispersăo para a interaça}

Da definição de matriz de dispersão, tem-se

$$
\begin{aligned}
& D(\hat{\delta})=E\left[[\hat{\delta}-E(\hat{\delta})][\hat{\delta}-E(\hat{\delta})]^{\prime}\right\} \\
& D e(a .92) \text {, tem-se que } \hat{\delta}-E(\hat{\delta})=P_{x} \varepsilon \text {, logo } \\
& D(\hat{\delta})=E\left[P_{x} \varepsilon \varepsilon^{\prime} P_{I}^{\prime}\right]=P_{x} E\left(\varepsilon \varepsilon^{\prime}\right) P_{x}^{\prime}=P_{x} \Sigma P_{I}^{\prime}
\end{aligned}
$$

Com a finalidade de se avaliar o produto $P_{I} \Sigma$ $P_{I}$, inicialmente, estudou-se a matriz $P_{I}$ atraves de suas componentes, considerando o exemplo inicial de (3.1). Como em IEMMA (1981), as generalizaçชes em termos de número de tratamentos principais e secundarios, foram apenas parciais, pois ficaram restritas ao esquena de PBIB, adotado no exemplo, isto $e$, a outros esquemas correspondem outras constituiç̧es matriciais. No entanto, o resultado final não se altera.

Desse modo, estudando as matxizes que comprem $P_{1}$ tem-se: 
$v \mathrm{E}_{1}\left(X_{1} X_{1}\right)^{-1} X_{1}=\frac{1}{b k u} v E_{1} X_{1}^{\prime}=\frac{1}{b k u} v E_{b k u}=\frac{1}{v u r} v{ }^{E_{b k u}}$

$$
\begin{aligned}
& r=1 \quad r=2 \\
& L^{-1} S^{\prime} R^{-1} X_{3}^{\prime}=\frac{1}{r u}\left[\begin{array}{llll|llll}
E & \phi & \phi & \phi & E & \phi & \phi & \phi \\
\phi & E & \phi & \phi & \phi & \phi & E & \phi \\
\phi & \phi & E & \phi & \phi & E & \phi & \phi \\
\phi & \phi & \phi & E & \phi & \phi & \phi & E
\end{array}\right]_{v r u}
\end{aligned}
$$

onde: $E={ }_{u} E_{u} E$ uma matriz de uns, de dimensరes (u) $\times(u)$; $\phi={ }_{u}{ }_{u}$ e uma matriz de zeros, de dimensoes $(u) \times(u)$;

$$
r=1 \quad r=2
$$

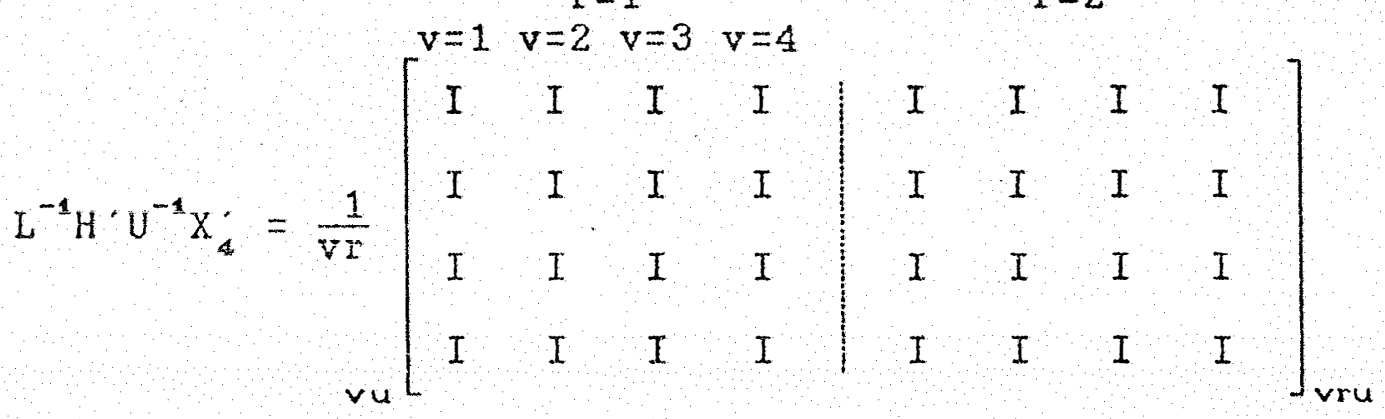

ou simplesmente,

$$
L^{-1} H^{\prime} U^{-1} X_{2}=\frac{1}{v r} v_{u} E_{b k u} \otimes I_{u}
$$

onde, $I=I_{u}$ é uma matriz identidade, de dimensós $(u) \times(u)$.

$$
\begin{aligned}
& r=1 \quad r=2 \\
& L^{-1} X_{5}=\frac{1}{r}\left[\begin{array}{llll}
I & \phi & \phi & \phi \\
\phi & I & \phi & \phi \\
\phi & \phi & I & \phi \\
\phi & \phi & \phi & I
\end{array}\left[\begin{array}{llll}
I & \phi & \phi & \phi \\
\phi & \phi & I & \phi \\
\phi & I & \phi & \phi \\
\phi & \phi & \phi & I
\end{array}\right]_{V r u}\right.
\end{aligned}
$$

Desse modo, ode-se formar as seguintes subratrizes; de dimensão ( $u$ x u ):

$\Pi_{1}=\frac{1}{r} I_{u}-\frac{1}{u r} E_{u}-\frac{1}{v r} I_{u}+\frac{1}{\operatorname{vur}} \mathrm{u}_{u}$ 
60.

$$
\begin{aligned}
& \Pi_{1}=\frac{v-1}{v u r}\left[\begin{array}{cccc}
u-1 & -1 & \cdots & -1 \\
-1 & u-1 & \cdots & -1 \\
\cdots & \cdots & \cdots & \cdots \\
-1 & -1 & \cdots & u-1
\end{array}\right]_{u} \\
& \Pi_{2}=\phi_{u}-\frac{1}{u r} \phi_{u}-\frac{1}{v r} I_{u}+\frac{1}{v u r} u_{u} \\
& \Pi_{2}=\frac{-1}{v u r}\left[\begin{array}{ccccc}
u-1 & -1 & \cdots & -1 \\
-1 & u-1 & \cdots & -1 \\
\cdots & \cdots & \cdots & \cdots \\
-1 & -1 & \cdots & u-1
\end{array}\right]_{u}
\end{aligned}
$$

Assim, a matriz $P_{I}$ ficou constituida do seguinte modo:

$$
\begin{aligned}
& r=1 \quad r=2 \\
& \mathrm{P}_{\mathrm{r}}=\left[\begin{array}{llll|llll}
\Pi_{1} & \Pi_{2} & \Pi_{2} & \Pi_{2} & \Pi_{1} & \Pi_{2} & \Pi_{2} & \Pi_{2} \\
\Pi_{2} & \Pi_{1} & \Pi_{2} & \Pi_{2} & \Pi_{2} & \Pi_{2} & \Pi_{1} & \Pi_{2} \\
\Pi_{2} & \Pi_{2} & \Pi_{1} & \Pi_{2} & \Pi_{2} & \Pi_{1} & \Pi_{2} & \Pi_{2} \\
\Pi_{2} & \Pi_{2} & \Pi_{2} & \Pi_{1} & \Pi_{2} & \Pi_{2} & \Pi_{2} & \Pi_{1}
\end{array}\right]_{v r u}
\end{aligned}
$$

Mas, verifica-se gue

$P_{x} \Sigma=\sigma_{b}^{2} P_{x}$

então, (a.95) fica

$$
D(\hat{\delta})=\sigma_{\mathrm{b}}^{2} \mathrm{P}_{\mathrm{x}} \mathrm{P}_{\mathrm{I}}
$$

onde, $\mathrm{P}_{\mathrm{X}} \mathrm{P}_{\mathrm{I}}^{\prime}$ é constituida pelas seguintes submatrizes:

(i) na diagonal principal:

$$
\Pi_{1} \Pi_{1}^{\prime}+3 \Pi_{2} \Pi_{2}^{\prime}+\Pi_{1} \Pi_{1}^{\prime}+3 \Pi_{2} \Pi_{2}^{\prime}=2 \Pi_{1} \Pi_{1}^{\prime}+2 \times 3 \Pi_{2} \Pi_{2}^{\prime}
$$

generalizando-se, tem-se

$$
r \Pi_{1} \Pi_{1}^{\prime}+r(v-1) \Pi_{2} \Pi_{2}^{\prime}=\frac{(v-1)}{v u r}\left[\begin{array}{cccc}
u-1 & -1 & \ldots & -1 \\
-1 & u-1 & \ldots & -1 \\
\ldots & \ldots & \ldots & \ldots \\
-1 & -1 & \ldots & u-1
\end{array}\right]_{u}
$$


(ii) submatriz fora da diagonal:

$$
\Pi_{1} \Pi_{2}^{\prime}+\Pi_{2} \Pi_{1}^{\prime}+\Pi_{2} \Pi_{2}^{\prime}+\Pi_{2} \Pi_{2}^{\prime}+\Pi_{1} \Pi_{2}^{\prime}+\Pi_{2} \Pi_{2}^{\prime}+\Pi_{2} \Pi_{1}^{\prime}+\Pi_{2} \Pi_{2}^{\prime}
$$

mas, como $\Pi_{1} \Pi_{2}^{\prime}=\Pi_{2} \Pi_{1}^{\prime}$, tem-se que

$$
2 \Pi_{1} \Pi_{2}^{\prime}+2 \Pi_{2} \Pi_{2}^{\prime}+2 \Pi_{1} \Pi_{2}^{\prime}+2 \Pi_{2} \Pi_{2}^{\prime}=2 r \Pi_{1} \Pi_{2}^{\prime}+2 r \Pi_{2} \Pi_{2}^{\prime}
$$

generalizando-se, tem-se

$$
2 r \Pi_{1} \Pi_{2}^{\prime}+r(v-2) \Pi_{2} \Pi_{2}^{\prime}=\frac{1}{v u r}\left[\begin{array}{cccc}
-(u-1) & 1 & \cdots & 1 \\
1 & -(u-1) & \cdots & 1 \\
\cdots & \cdots & \cdots & \cdots \\
1 & 1 & \cdots & -(u-1)
\end{array}\right]_{u}
$$

\subsection{Análise da variancia}

3.7.1. Somas de quadrados

De acordo com o modelo linear $y=x \theta+\varepsilon$, sabe-se que a soma de quadrados do residuo e dada por

$$
\text { SQRes }=\hat{\varepsilon} \cdot \hat{\varepsilon}=y^{\prime} y-\hat{\theta}^{\prime} x^{\prime} y
$$

onde: $y^{\prime} y \in$ definida como a soma de quadrados total, representada por SQTot; e

$\hat{\theta}^{\prime} X \cdot y$ é definida como a soma de quadrados de parametros ou a redução devida aos parametros, representada por SQP, e dada por:

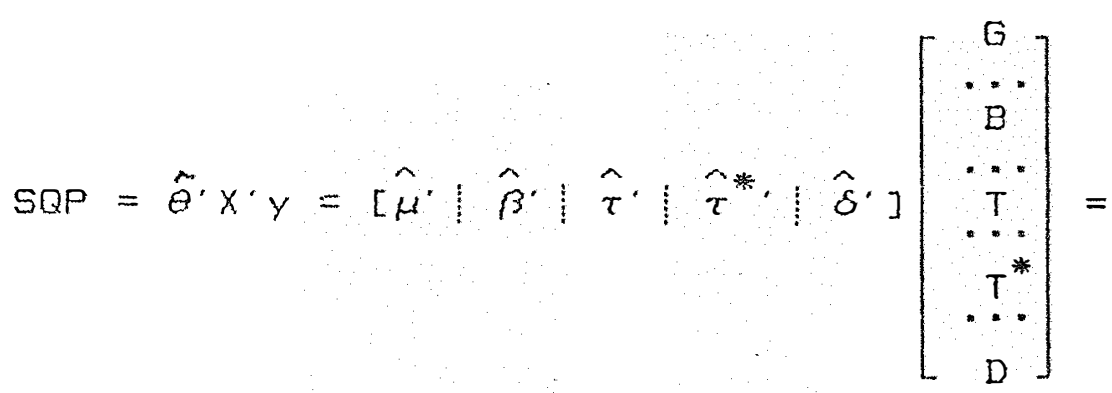

$S Q P=\hat{\mu} G+\hat{\beta^{\prime}} B+\hat{\tau} \cdot T+\hat{\tau} \cdot T^{*}+\hat{\mathcal{E}^{\prime}} \cdot D$.

$$
\text { Mas, de (a.10) } \hat{\beta}=K^{-1} B-K^{-1} N \cdot \hat{\tau}-b_{1} \hat{\mu}_{3}
$$

ent

$$
\begin{aligned}
& \operatorname{SQP}=\hat{\mu G}+\hat{\tau} \cdot T+\left(K^{-1} B-K^{-1} N \cdot \hat{\tau}-b_{1} \hat{\mu}\right) \cdot B+\hat{\tau}^{*} \cdot T^{*}+\hat{\mathcal{G}} \cdot \mathrm{D}
\end{aligned}
$$

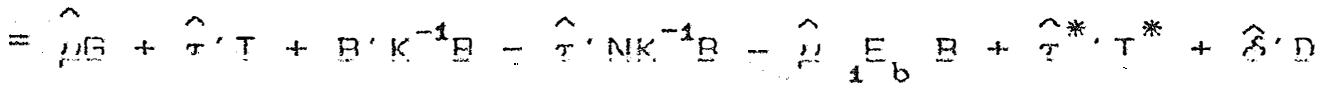




$$
\begin{aligned}
& S Q P=\hat{\mu G}+\hat{\tau} \cdot\left(T-N K^{-1} B\right)+B \cdot K^{-1} B-\hat{\mu} E_{b} B+\hat{\tau} \cdot T^{*}+\hat{\delta} \cdot D \\
& =\hat{\mu G}+\hat{\tau} \cdot Q+\left(B \cdot K^{-1} B-\hat{\mu} G\right)+\hat{\tau}^{*} \cdot T^{*}+\hat{\delta} \cdot D \\
& \text { Mas, ainda, tem-se que } \\
& \hat{\imath}^{*} \cdot T^{*}=\left(U^{-1} T^{*}-\mathrm{u}_{1} \mu\right) \cdot T^{*}=T^{*} \cdot U^{-1} T^{*}-\mu_{1} E_{u} T^{*}= \\
& =T^{*} \cdot U^{-1} T^{*}-\mu G \\
& \hat{\delta} D=\left(L^{-1} D-L^{-1} S \cdot R^{-1} T-L^{-1} H U^{-1} T^{*}+v_{u} E_{1} \mu\right) \cdot D= \\
& =D \cdot L^{-1} D-T R^{-1} S L^{-1} D-T^{*} U^{-1} H L^{-1} D+H_{V u} D
\end{aligned}
$$

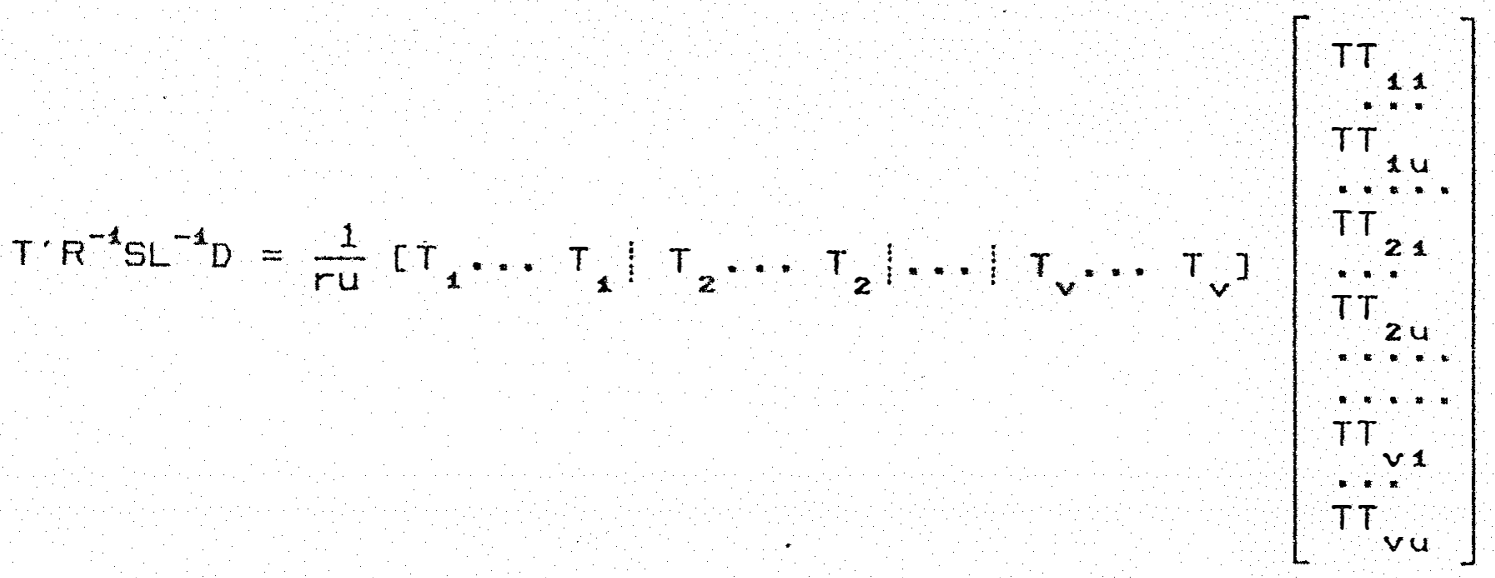

$$
\begin{aligned}
& =\frac{1}{r u}\left(T_{1}^{2}+\cdots+T_{v}^{2}\right)
\end{aligned}
$$$$
T^{*} \cdot U^{-1} H L^{-1} D=\frac{1}{v r}\left[T_{1}^{*} \cdots T_{u}^{*} T_{1}^{*} \cdots T_{u}^{*} \cdots T_{1}^{*} \cdots T_{u}^{*}\right]
$$$$
\left[\begin{array}{c}
T T_{1} \\
T T_{1 u} \\
T T_{21} \\
T T_{2 u} \\
T_{V 1} \\
T T_{v u}
\end{array}\right]
$$$$
T^{*} \cdot U^{-1} H L^{-1} D=\frac{1}{V r}\left(T_{1}^{* 2}+\cdots+T_{u}^{* 2}\right)
$$$$
\text { Mas, como } S L^{-1} D=T, H L^{-1} D=T^{*} e_{x_{V u}} D=G \text {, }
$$

então.

$\hat{\delta} D=D^{\prime} L^{-B} D-T \cdot R^{-1} T-T^{\prime \prime} U^{-1} T^{*}+\hat{H G}$ 
63.

$$
\begin{aligned}
& \hat{\delta}^{\prime} D=\left(D \cdot L^{-1} D-\hat{\mu} G\right)-\left(T R^{-1} T-\hat{\mu} G\right)-\left(T^{*} U^{-1} T^{*}-\hat{\mu} G\right) \\
& \text { Desse modo, (b.3) resultou em } \\
& S U P=\hat{\mu G}+\hat{\tau} Q+\left(B \cdot K^{-1} B-\hat{\mu} G\right)+T^{*} \cdot U^{-1} T^{*}-\hat{H} G+ \\
& +\left(D \cdot L^{-1} D-\hat{\mu} G\right)-\left(T \cdot R^{-1} T-\hat{\mu} G\right)-\left(T^{*} U^{-1} T^{*}-\hat{\mu G}\right)
\end{aligned}
$$

onde:

$$
\begin{aligned}
& \hat{\mu G}=\operatorname{SQC} \text { \& a soma de quadrados da correçã ; } \\
& \hat{\tau} \cdot Q=\operatorname{SQT}(a j) \text { e a soma de quadrados de tratamentos princi- } \\
& \text { pais ajustada para blocos; } \\
& B^{\prime} K^{-1} B-\hat{H} G=S Q B \text { é a soma de quadrados de blocos, obtida de } \\
& \hat{\tau}^{*} \cdot T^{*}=T^{*} \cdot U^{-1} T^{*}-\hat{H G}=S Q T^{*} \text { e a soma de quadrados de trata- } \\
& \text { mentos secundários, obtida de maneira usual; } \\
& \hat{\tau} \cdot T=T^{\prime} R^{-1} T-\hat{\mu} G=S Q T \in \text { a soma de quadrados de tratamentos } \\
& \text { principais, obtida de maneira usual, sem o ajuste para } \\
& \text { blocos; }
\end{aligned}
$$

$D \cdot L^{-1} D-\hat{H} G-\hat{\tau} \cdot T-\hat{\tau}^{*} T^{*}=S Q I$ e a soma de quadrados da interação tratamentos principais $x$ tratamentos secundarios, obtida de maneira usual.

\subsubsection{Soma de quadrados de residero}

De acordo com o modelo adotado em (a.1), a soma de quadrados de residuo pode ser particionada, de maneira conveniente, em

$$
\text { SQRES }=\operatorname{SQR}(a)+\operatorname{SQR}(b)
$$

Reportando-se a (a.1), tem-se que o modelo de parcelas subdivididas pressupóse que o erro aleatório de cada observação ( $x_{i j l}$ ), pode ser decomposto em duas fontes independentes de erros: $\eta_{i j} e e_{i j l}$, onde $e_{i j l} e 0$ termo do erro a nivel de subparcelas e $\eta_{i j}$ é o termo do erro especifico da medida efetuada no tratamento $i$ no bloco j, ou simplesmente, \& o erro a nivel de parcelas. De acordo com CHACKRABARTI $(1962)$, tem-se

$$
e_{i j k}=y_{i j k}-H-b_{j}-t_{i}-t_{k}^{*}-\delta_{i k}-n_{i j}
$$

sendo que $\eta_{i j}$ agora se comporta como um parametro $\rightarrow$ maic do 
modelo, e para obter soluçós de minimos quadrados, deve-se tornar minima a seguinte soma de quadrados de erros

$$
\sum_{i j l} e_{i j l}^{2}=\sum_{i j l}\left(y_{i j l}-\mu-b_{j}-t_{i}-t_{i}^{*}-\delta_{i l}-n_{i j}\right)^{2}
$$

Minimizando a soma de quadrados acima, as solu- ç̃es de $n_{i j}$ podem ser obtidos através da seguinte expressão, na forma matricial,

$$
x_{\sigma} x_{1} \hat{\mu}+x_{\sigma} x_{2} \hat{\beta}+x_{\sigma} x_{3} \hat{\tau}+x_{\sigma} x_{\sigma} \hat{\eta}=x_{\sigma} y
$$

onde $X_{\sigma}=I_{(v r)} \otimes_{u} E_{1} e$ uma matriz dos coeficientes associados aos $\eta_{i j}$ de dimensores (vru) \& (vr).

$$
\begin{aligned}
& \text { Assim, uma solucão para } \eta \text { e } \\
& \hat{n}=x_{\sigma}^{+} y-x_{\sigma}^{+} P_{2} y-X_{\sigma}^{+}\left(I-P_{2}\right) x_{3} \hat{\tau} \\
& =x_{0}^{+}\left(I-P_{2}\right) y-X_{0}^{+}\left(I-P_{2}\right) x_{3} \hat{\tau}=x_{0}^{+}\left(I-P_{2}\right)\left(y-x_{3} \hat{\tau}\right)
\end{aligned}
$$

sendo $P_{2}=x_{2}\left(x_{2} x_{2}\right)^{-1} x_{2}$.

Desse modo, a soma de quadrados relativa a $n$, representada por SQR(a), é

$$
\begin{aligned}
& \operatorname{SQR}(a)=\hat{n}^{\prime} x \cdot y=y^{\prime} P_{\sigma} y-y^{\prime} P_{z} y-\hat{\tau} \cdot Q \\
& =\left(y \cdot P_{6} y-y \cdot P_{1} y\right)-\left(y \cdot P_{2} y-y \cdot P_{1} y\right)-\hat{\tau} Q \\
& =\operatorname{SQPar}-\operatorname{SQB}-\operatorname{SQTP}(a j)
\end{aligned}
$$

onde:

$$
\begin{aligned}
& P_{1}=x_{1} x_{1}^{+}=x_{1}\left(x_{1} x_{1}\right)^{-1} x_{1}, \quad P_{0}=x_{0} x_{0}^{+}=x_{0}\left(x_{0} x_{0}\right)^{-1} x_{0} ; \\
& \text { SQPar }=y^{\prime}\left(P_{\sigma}-P_{1}\right) y=\frac{1}{u} \sum_{i, j} y_{i j}^{2}-S-S Q C \text { e a soma de quadrados }
\end{aligned}
$$
de parcelas;

$S Q B=\left(y P_{2} Y-Y P_{1} Y\right) \in a$ soma de quadrados de blocos;

$\operatorname{SQT}(a j)=\hat{\tau} Q$ a soma de quadrados de tratamentos princi pais ajustada.

$$
\text { Sendo SQRes }=y^{\prime} y-\hat{\theta}^{\prime} x \cdot y=\operatorname{SQR}(a)+\operatorname{SQR}(b)
$$

então, de (b.5) e de (b.7), tem-se

$$
\begin{aligned}
\operatorname{SQR}(b) & =y^{\prime} y-S Q C-S Q P a r-S Q T^{*}-S Q I \\
& =y^{\prime} y-S Q C-S Q B-S Q T(a j)-S Q T^{*}-S Q I \\
& =y^{\prime}\left(I-P_{G}-P_{5}+P_{3}\right) y
\end{aligned}
$$

Qutro procedimento para a decomposição ou par-

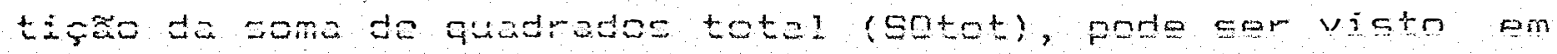


IEMMA (1981) e VIZONI (1984).

\subsubsection{Esperanca matematica das somas de quadradas}

Para a determinação das esperanças matematicas das formas quadraticas, que fornecem as somas. de quadrados, usou-se o seguinte teorema auxiliar, enunciado em SEARLE ( 1971$)$ :

TEDREMA - Quando y é $N(X \theta, \Sigma)$, então

$$
E\left(y^{\prime} P y\right)=\operatorname{tr}(P \Sigma)+\theta^{\prime} x \cdot P X \theta \text {. }
$$

sendo: $P$ o núcleo da forma quadrática;

tr indica o operador traço de uma matriz;

$\Sigma=\operatorname{Var}(y)$ é a matriz de covariancias, de (a.72).

\subsubsection{Esperanca matematica de SQC}

$$
\begin{aligned}
\text { Sabe-se que, } \hat{\mu}=\left(x_{1}^{\prime} x_{1}\right)^{-1} x_{1} y \text { e } G=x_{1}^{\prime} y, & \text { ent芯o } \\
\text { SQC } \left.=\hat{\mu} G=y^{\prime} x_{1}\left(x_{1} \cdot x_{1}\right)^{-1} x_{1} y=y^{\prime} P_{1}\right), & \text { (b.10 })
\end{aligned}
$$

sendo $P_{1}=\frac{1}{v r u}$ vru $E_{v r u}$, cujo $E$ é uma matriz de uns.

$$
\text { Aplicando (b. q) em (b.10), tem-se }
$$

$E(S Q C)=E\left(Y \cdot P_{1} Y\right)=\operatorname{tr}\left(P_{1} V\right)+\theta \cdot X \cdot P_{1} X \theta$

$$
\text { Mas, } P{ }_{1} \Sigma=\left(\sigma_{b}^{2}+u \sigma_{a}^{2}\right) P_{1}
$$

como $P_{1}$ \& uma matriz simetrica e idempotente; tem-se que $\operatorname{tr}\left(P_{1} \Sigma\right)=r\left(P_{1} \Sigma\right)=\left(\sigma_{b}^{2}+4 \sigma_{a}^{2}\right) r\left(P_{1}\right)$

mas, $r\left(P_{1}\right)=1$, 1090

$$
\operatorname{tr}\left(P_{1} \Sigma\right)=\left(\sigma_{b}^{2}+4 \sigma_{a}^{2}\right)
$$

$\theta \cdot X \cdot P_{1} X \theta=\theta \cdot x \cdot x_{1}\left(x_{1}^{\prime} x_{1}\right)^{-1} x_{1} \times \theta$,

$$
\text { mas, }
$$$$
\begin{aligned}
x_{1} x_{\theta} & =x_{1}^{\prime} x_{1} \mu+x_{1} x_{2} \beta+x_{1} x_{1} \tau+x_{1} x_{4} \tau+x_{1} x_{5} \delta \\
& =n \mu+r u{ }_{1} E_{v} \tau+k u_{1} E_{b} \beta+v r{ }_{1} E_{u} \tau+r{ }_{1} E_{v u} \delta
\end{aligned}
$$

aplicando-se as restriçóes impostás em (a.8), resulta-se em

$$
x_{1}^{\prime} \times \theta=n \mu
$$

logo,

$\theta \cdot x \cdot P_{i} x \theta=\mu n\left(x_{i} x_{i}\right)^{-1} \Pi \mu=\mu n \mu=\mu_{\mu}^{2}$ 


\section{Portanto,}

$$
E(S Q C)=E(\hat{\mu} G)=\pi \mu^{2}+\left(\sigma_{b}^{2}+u \sigma_{a}^{2}\right)
$$

\subsubsection{Esperança matemática de $\operatorname{sot}(a j)$}

Sabe-se de $(3.7 .1)$, que

$$
\operatorname{SQT}\left(a_{j}\right)=\hat{\tau} \cdot Q=Q \cdot M^{-1} Q=Q \cdot \Omega Q=Q^{\prime} C^{-} Q
$$

Seja de (a.80), a matriz

$W=\left[v_{v} \phi_{1}-N K^{-1} I_{v} \phi_{u}{ }_{v}^{\phi} \phi_{v u}\right] d e$ dimensठes $v \times$ vru.

Então,

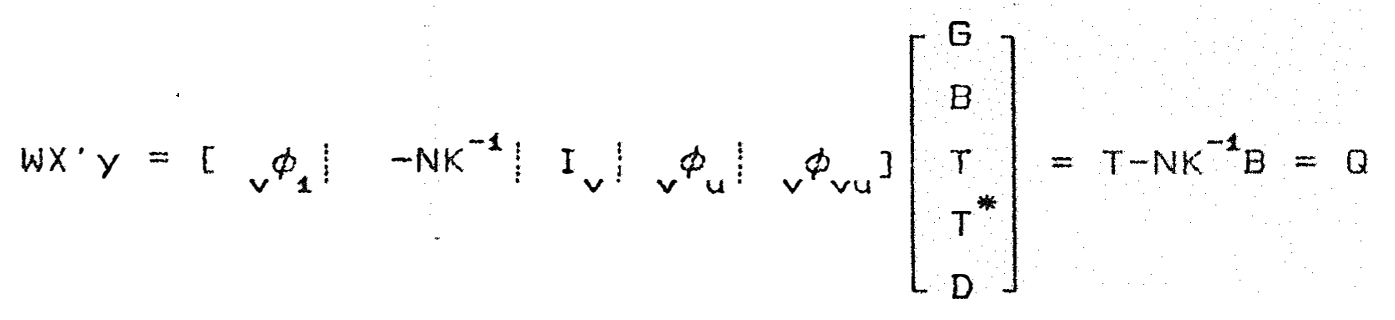

$$
\begin{aligned}
& \text { Substituindo-se em }(b .12) \text {, tem-se } \\
& \operatorname{SQT}(a j)=y \cdot x w^{\prime} C^{-} w X \cdot y=y \cdot P_{T^{\prime}} y^{\prime} \\
& \text { Utilizando o teorema de (b.9), tem-se } \\
& E[\operatorname{SQT}(a j)]=E\left(y \cdot P_{\mathbf{T}} y\right)=\operatorname{tr}\left[P_{\mathbf{r}} \Sigma\right]+\theta^{\prime} x \cdot P_{\mathbf{T}} x \theta
\end{aligned}
$$

Mas, $W X \cdot \Sigma=\left(X_{2}-N K^{-1} X_{a}\right) \Sigma=\left(\sigma_{b}^{2}+u \sigma_{a}^{2}\right) W X^{.}$

logo,

$\operatorname{tr}\left(P_{T} V\right)=\operatorname{tr}(X W \cdot \operatorname{XN} X)\left(\alpha_{b}^{2}+u \alpha_{a}^{2}\right) \cdot$ Se as dimenşes sao favoráveis, entao $\operatorname{tr}\left(X W^{\prime} \Omega W X^{\prime}\right)=\operatorname{tr}\left(\Omega W X^{\prime} X W^{\prime}\right)$. Mas,

$$
\begin{aligned}
W X^{\prime} X W^{\prime} & =\left(X_{2}-N K^{-1} x_{3}^{\prime}\right)\left(X_{2}-N K^{-1} x_{3}^{\prime}\right)^{\prime}= \\
& =X_{2}^{\prime} X_{2}-X_{2}^{\prime} X_{3} K^{-1} N^{\prime}-N K^{-1} X_{3}^{\prime} X_{2}+N K^{-1} X_{3}^{\prime} X_{3} K^{-1} N^{\prime} \\
& =R-N K^{-1} N^{\prime}=C
\end{aligned}
$$

ent注, $\left.\operatorname{tr}\left(X W^{\prime} \Omega W^{\prime}\right)=\operatorname{tr}(\Omega)^{\prime}\right)$. Como $\Omega$ e uma matriz inversa generalizada (condicional) de $C$, entao verifica-se a propriedade $C \Omega=C$, e $\Omega C$ e idempotente. Assim,

$$
\begin{aligned}
& \operatorname{tr}\left(P \sum\right)=\left(\sigma_{b}^{2}+u \sigma_{a}^{2}\right) r(\Omega C)=\left(\sigma_{b}^{2}+u \sigma_{a}^{2}\right) r(C) \\
& =\left(\sigma_{b}^{2}+4 \sigma_{a}^{2}\right)(v-1) \\
& \text { Tem-se tamberm, que }
\end{aligned}
$$

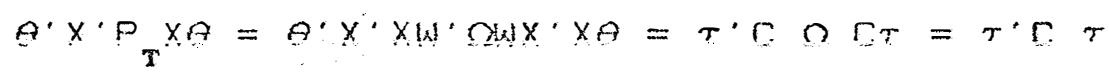


portanto,

$$
E(\operatorname{SQT}(a j))=\tau C \tau+\left(\sigma_{b}^{2}+u \sigma_{a}^{2}\right)(v-1) \sigma^{2}
$$

Do posto de C, obtem-se o número de graus de liberdade para tratamentos principais; ou usando a seguinte definição, dada por IEMMA (1987):

DEF INIŞÃO - Define-se o número de graus de liberdade de uma soma de quadrados, como o posto da matriz da forma quadratica correspondente.

\subsubsection{Esperanca matematica de SQB}

Do item $(3.7 .1)$, tem-se que SQB $=B^{\prime} K^{-1} B-\hat{\mu} G$. Sendo,

$B \cdot K^{-1} B=y^{\prime} x_{2} K^{-1} x_{2} Y=y^{\prime} P_{2} y$

entzo, aplicando-se o teorema de $(0.9)$, tem-se

$E\left(B \cdot K^{-1} B\right)=E\left(Y \cdot P_{2} y\right)=\operatorname{tr}\left(P_{2} \Sigma\right)+\theta \cdot x \cdot P_{2} \times \theta$

mas, $P_{2} \Sigma=\left(\sigma_{b}^{2}+u \sigma_{a}^{2}\right) P_{2}$, entao

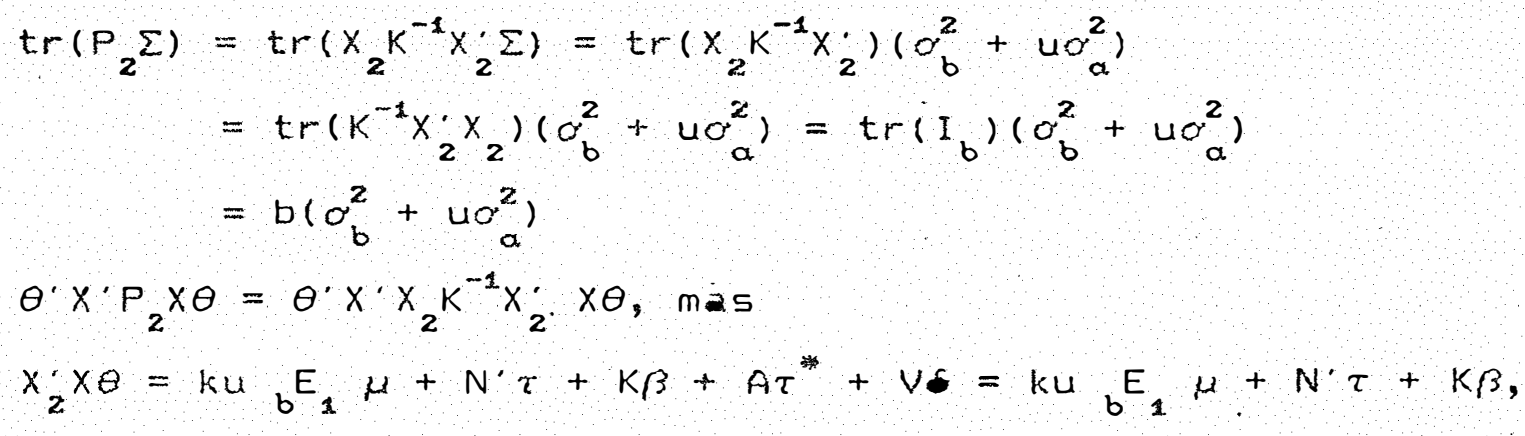

impondo-se as restriçoses definidas en $(a .8)$, tem-se

$\theta \times P_{2} \times \theta=\left(k u b_{b} E_{1} \mu+N T+K \beta\right) K^{-1}\left(k u_{b} E_{1} \mu+N T+K \beta\right)$

Efetuando-se as multiplicaçoses e aplicando as

restriçठes de $(a .8)$, obteve-se

$\theta^{\prime} X \cdot P_{2} X \theta=\pi \mu^{2}+2 \beta^{\prime} N^{\prime} \tau+\beta^{\prime} K \beta+\tau^{\prime} N K^{-1} N^{\prime} \tau$

$=\pi \mu^{2}+2 \beta^{\prime} N^{\prime} \tau+\beta^{\prime} K \beta+\tau \cdot R \tau-\tau \cdot C \tau$

Desse modo;

$E\left(B^{\prime} K^{-1} B\right)=n \mu^{2}+2 \beta^{\prime} N^{\prime} \tau+\beta^{\prime} K \beta+\tau \cdot R \tau-\tau \cdot C \tau+$

$+b\left(\sigma_{b}^{2}+u \sigma_{a}^{2}\right)$

Logo, $E\left(S(2 B)=E\left(B \cdot K^{-1} B\right)-E(\hat{\mu} G)\right.$. 
68.

Assim, de (b.11) e de $(b .18)$, tem-se que

$E(S Q B)=2 \beta^{\prime} N^{\prime} \tau+\beta^{\prime} K \beta+\tau^{\prime} R \tau-\tau \cdot C \tau+(b-1)\left(\sigma_{b}^{2}+4 \sigma_{a}^{2}\right)(b .19)$

\subsubsection{Esperanca matematica de SQT}

Do item $(3.7 .1)$, tem-se que SQT $T^{*}=\tau^{*} \cdot T^{*}$.

Sendo,

ent药,

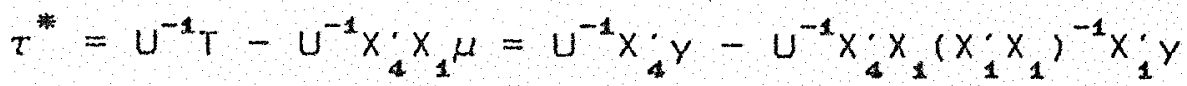

$\left.\tau^{*} \cdot T^{*}=y^{\prime}\left[x_{4} U^{-1} X_{4}-X_{1}\left(X_{1} x_{1}\right)^{-1} x_{1} x_{4} U^{-1} x_{4}\right] y=y P_{4}-P_{1} P_{4}\right] Y$,

onde, $P_{4}=\frac{1}{v r}\left[E_{v r} E_{v r} I_{u}\right]$,

Facilmente, verifica-se que $P_{1} P_{4}=P_{1}$, então,

sendo

$$
S Q T^{*}=Y^{\prime}\left(P_{4}-P_{1}\right) Y=\tau \cdot T^{*}=Y \cdot P_{S} Y
$$

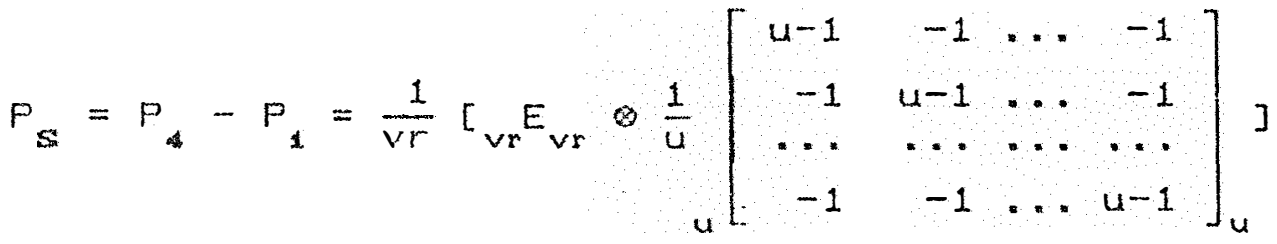

$$
\begin{aligned}
& E\left(y \cdot P_{B} y\right)=\operatorname{tr}(P \Sigma)+\theta \cdot x \cdot P_{B} X \theta \\
& \text { Mas, verifica-se que } P_{s} \Sigma=\sigma_{b}^{2} P_{s}
\end{aligned}
$$

então,

$$
\operatorname{tr}(P \Sigma)=\operatorname{tr}\left(P_{s}\right) \sigma_{b}^{2}
$$

Mas, sendo $P_{8}$ uma matriz simétrica e idempo-

tente, tem-se que

$$
\begin{aligned}
& \operatorname{tr}\left(P_{s} \Sigma\right)=\sigma_{b}^{2} r\left(P_{s}\right)=\sigma_{b}^{2}(u-1) \\
& \theta^{\prime} X^{\prime} P_{8} X \theta=\theta^{\prime} x^{\prime}\left[x_{4} U^{-1} x_{4}^{\prime}-x_{1}\left(x_{1} x_{1}^{\prime}\right)^{-1} x_{1}^{\prime}\right] \times \theta \\
& \text { mas, } x_{4} x_{\theta}=x_{4} x_{4} \mu+x_{4}^{\prime} x_{2}^{\tau}+x_{4} x_{3} \beta+x_{4}^{\prime} x_{4}^{\tau}+x_{4}^{\prime} x_{5} \delta \\
& \text { Impondo-se as restriços de }(a .8) \text { tem-se que } \\
& x_{4} \cdot x^{\prime}=x_{4} x_{4} y+x_{4} x_{4} \tau^{*}
\end{aligned}
$$

Ent花

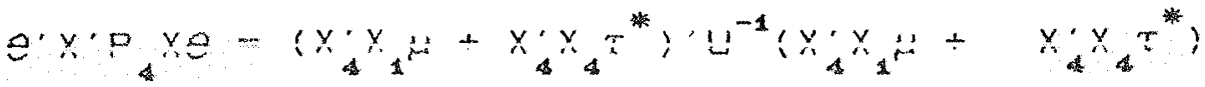


fazendo as multiplica̧̧̋es e aplicando restriç̧es de (a.8), obtem-se

$$
\theta^{\prime} \times P_{A} \times \theta=n \mu^{2}+\tau^{*} \cdot u \tau^{*}
$$

Portanto, de $(b .22)$ e $(b .11)$, tem-se que

$$
\begin{array}{r}
E\left(S Q T^{*}\right)=n H^{2}+\tau^{*} U \tau^{*}+\sigma_{b}^{2}(u-1)-n \mu^{2} \text { ou } \\
E\left(S Q T^{*}\right)=\tau^{*} u \tau^{*}+(u-1) \sigma_{b}^{2}
\end{array}
$$

\subsubsection{Esperanca matematica de SQI}

Do item $(3.7 .1)$, tem-se que

$$
\begin{aligned}
& S Q I=\hat{\delta} \cdot D=D \cdot L^{-1} D-T \cdot R^{-1} T-T^{*} U^{-1} T+G^{2} / n
\end{aligned}
$$

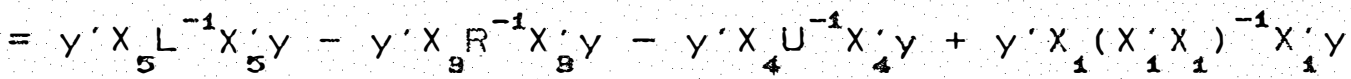

$$
\begin{aligned}
& =y^{\prime} P_{5} Y-y^{\prime} P_{3} Y-y^{\prime} P_{4}+y^{\prime} P_{1} Y
\end{aligned}
$$

Desenvolvendo por partes, de $(0.9)$, vem que

$$
E\left[Y P_{5} Y\right]=\operatorname{tr}\left(P_{5} \Sigma\right)+\theta \cdot x \cdot P_{5} \times \theta
$$

mas, $\operatorname{tr}\left(P_{5} \Sigma\right)=r\left(P_{5} \Sigma\right)=\frac{1}{r} \operatorname{vru}\left(\sigma_{a}^{2}+\sigma_{b}^{2}\right)=\operatorname{vu}\left(\sigma_{a}^{2}+\sigma_{b}^{2}\right)$

$$
X_{5} X \theta=r_{V u} E_{1} \mu+V \beta+S^{\prime} \tau+H \tau^{*}+L S
$$

$\theta^{\prime} X P_{5} \times \theta=\left[r_{V U} E_{1} \mu+V^{\prime} \beta+S^{\prime} \tau+H \cdot \gamma^{*}+L \delta\right]^{-1}\left[r_{V u} E_{1} H+V \cdot \beta+S^{\prime} \tau+H^{\prime} \tau^{*}+L \delta\right]$

$$
\begin{aligned}
& =\mu r_{1}^{2} E_{v u} L_{v u}^{-1} E_{1}+2 \mu r_{1} E_{v u} L^{-1} v \cdot \beta+ \\
& +2 \mu r E_{v u} L^{-1} S^{\prime} \tau c+2 \mu r_{1} E_{V u} L^{-1} H^{\prime} \tau^{*}+ \\
& +2 \mu{ }_{\alpha} E_{V u} L^{-1} L \delta+\beta N R^{-1} N \beta+2 \beta V L^{-1} S^{\prime} \tau+ \\
& +2 \beta^{\prime} V L^{-1} H^{\prime} \tau^{*}+2 \beta V \delta+\tau^{\prime} S L^{-1} S^{\prime} \tau+2 \tau S L^{-1} H^{\prime} \tau^{*}+ \\
& +2 \tau^{\prime} S \delta+\tau^{*} \cdot H L^{-1} H \tau^{*}+2 \tau^{*} H \delta+\delta \cdot L \delta
\end{aligned}
$$

impondo-se as restriçzes de $(a .8)$, e simplificando, tem-se $\theta^{\prime} \times P_{5} \times \theta=n \mu^{2}+\beta^{\prime} N^{\prime} R^{-1} N \beta+2 \beta^{\prime} N^{\prime}+\tau R \tau+\tau * U \tau+\mathcal{L} \cdot L \delta$

portanto,

$$
\begin{aligned}
& E\left[y^{\prime} P_{5} Y\right]=n \mu^{2}+\beta^{\prime} N^{\prime} R^{-1} N \beta+2 \beta^{\prime} N^{\prime} \tau+\tau^{\prime} R \tau+\tau^{*} \cdot U \tau^{*}+ \\
& +\delta \cdot L \delta+v u\left(\sigma_{a}^{2}+\sigma_{b}^{2}\right) \\
& E\left[Y^{\prime} P_{3} Y\right]=\operatorname{tr}\left(P_{3} \Sigma\right)+\theta \cdot X^{\prime} P_{\mathbf{3}} X \theta \\
& \operatorname{tr}\left(P_{3} \Sigma\right)=\operatorname{tr}\left(P_{3}\right)\left(\sigma_{b}^{2}+u \sigma_{a}^{2}\right)=\operatorname{tr}\left(x_{3} R^{-1} x_{3}\right)\left(\sigma_{b}^{2}+u \sigma_{a}^{2}\right)= \\
& =\operatorname{tr}\left(R^{-1} X_{3} X_{3}\right)\left(\sigma_{b}^{2}+u \sigma_{\alpha}^{2}\right)=\operatorname{tr}\left(I_{v}\right)\left(\sigma_{b}^{2}+u \sigma_{a}^{2}\right)=
\end{aligned}
$$




$$
\begin{aligned}
& \operatorname{tr}\left(P_{a} \Sigma\right)=v\left(\sigma_{b}^{2}+u \sigma_{a}^{2}\right) \\
& x_{9} x^{\theta}=x_{3} x_{1} \mu+x_{9} x_{2} \beta+x_{3} x_{3} \tau+x_{9} x_{4} \tau^{*}+x_{3} x_{5} \delta \\
& =r E_{1} E_{V} \mu+N \beta+R \tau \\
& \theta \cdot X \cdot P_{\mathbf{g}} X \theta=\left[r u_{1} E_{V} \mu+N \beta+R \tau\right] \cdot R^{-1}\left[r U_{1} E_{V} \mu+N \beta+R T\right] \\
& =\mu r^{2} u^{2} E_{v} R^{-1} E_{1} \mu+2 \mu r u E_{v} R^{-1} N \beta+ \\
& +2 \mu r u E_{V u} R^{-1} R T+\beta N^{\prime} R^{-1} N \beta+2 \beta N R^{-1} R \tau+\tau R T \\
& =\pi \mu^{2}+\beta \cdot R^{-1} N \beta+2 \beta \cdot N \cdot \tau+R \tau
\end{aligned}
$$

portanto,

$E\left(y^{\prime} P_{g} Y\right)=n \mu^{2}+\beta^{\prime} N^{\prime} R^{-1} N \beta+2 \beta^{\prime} N^{\prime} \tau+\tau \cdot R \tau+v\left(\sigma_{b}^{2}+u \sigma_{a}^{2}\right)(b .27)$

$E\left(Y^{\prime} P_{4} Y\right)=\operatorname{tr}\left(P_{4} \Sigma\right)+\theta \cdot x \cdot P_{4} x \theta$

como

$\operatorname{tr}\left(P_{d} \Sigma\right)=\frac{1}{v r} \operatorname{tr}\left(\dot{v r}_{v r} E_{u} U_{u}\right) \Sigma=\frac{1}{v r} \operatorname{vru}\left(\sigma_{a}^{2}+\sigma_{b}^{2}\right)=u\left(\sigma_{a}^{2}+\sigma_{b}^{2}\right)$

então re (b.23), tem-se

$$
E\left(y \cdot P_{4} y\right)=n \mu^{2}+\tau^{*} \cdot u \tau^{*}+u\left(\sigma_{a}^{2}+\alpha_{b}^{2}\right)
$$

Desse modo, a esperança matemática da SQI fica $E(S Q I)=E\left[y^{\prime} P_{5} Y\right]-E\left[y^{\prime} P_{\mathbf{3}} y\right]-E\left[y^{\prime} P_{4} y\right]+E\left[y^{\prime} P_{3} Y\right]$ de $(b .26),(b .27),(b .28)$ e (b.11), obtem-se

$$
\begin{aligned}
E(S Q I)= & \delta^{\prime} L \delta+v u\left(\sigma_{a}^{2}+\sigma_{b}^{2}\right)-v\left(u \sigma_{a}^{2}+\sigma_{b}^{2}\right)-u\left(\sigma_{a}^{2}+\sigma_{b}^{2}\right)+\left(u \sigma_{a}^{2}+\sigma_{b}^{2}\right) \\
= & \delta^{\prime} L \delta+(v u-u-v+1) \sigma_{b}^{2} \\
& E(S Q I)=\delta^{\prime} L \delta+(v-1)(u-1) \sigma_{b}^{2}
\end{aligned}
$$

3.7.3.6. Esperança matematica de serot

Do item $(3.7 .1)$, tem-5e que

sendo,

$$
\text { SQTOt }=y^{\prime} y-\text { Cor }=y^{\prime} I_{\text {vru }} Y-y^{\prime} P_{1} Y
$$

$$
\begin{aligned}
E\left(y^{\prime} I_{v r u} y\right) & =\operatorname{tr}\left(I_{v r u} \Sigma\right)+\theta^{\prime} x^{\prime} I_{v r u} x \theta \\
& =\operatorname{tr}(\Sigma)+\theta^{\prime} x^{\prime} x \theta=\operatorname{vru}\left(o_{a}^{2}+\sigma_{b}^{2}\right)+\theta^{\prime} x \cdot x \theta
\end{aligned}
$$

$\theta^{\prime} x^{\prime} X \theta=\left[\mu x_{2}^{\prime}+\beta^{\prime} x_{2}^{\prime}+\tau^{\prime} x_{3}^{\prime}+\tau^{*} \cdot x_{4}^{\prime}+\delta \cdot x_{5}^{\prime}\right]\left[x_{4} \mu+x_{2} \beta+x_{9} \tau+x_{4} \tau^{*}+x_{5} \delta\right]$ fazendo-se as multiplicaçoses e impondo-se as restriços de $(a .8)$, obtem-se 
$\theta^{\prime} \times x \theta=n \mu^{2}+\beta^{\prime} K \beta+2 \beta \cdot N \tau+\tau \cdot R \tau+\tau^{*} \cdot U \tau+\delta^{*} L \delta$

então,

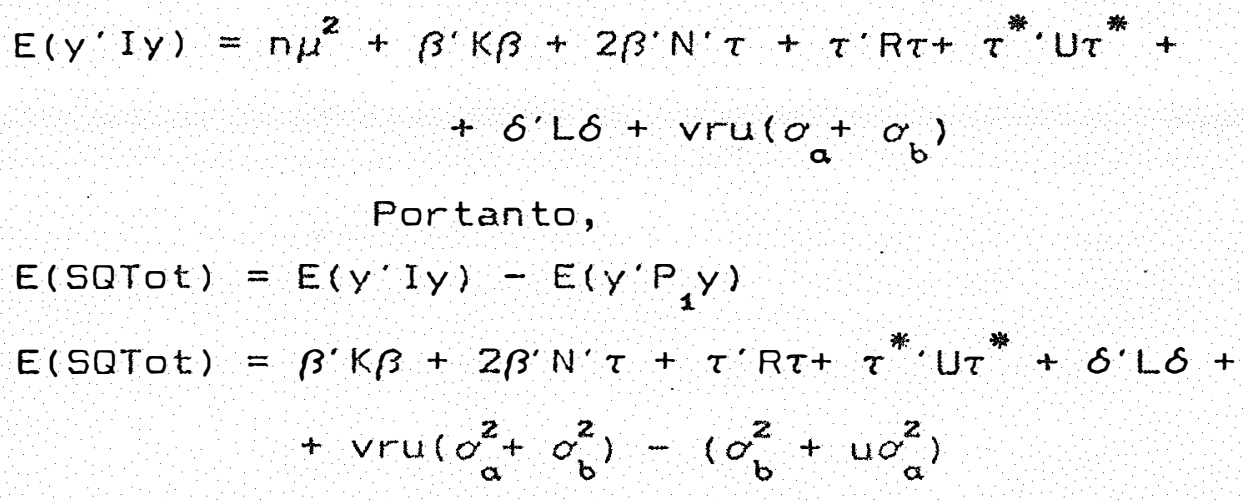

Portanto,

$E(S Q T O t)=E(Y \cdot I Y)-E\left(Y^{\prime} P_{1} Y\right)$

$E(S Q T O t)=\beta^{\prime} K \beta+2 \beta^{\prime} \tau+\tau R \tau+\tau^{*} U \tau^{*}+\delta L \delta+$

$$
+\operatorname{vru}\left(\alpha_{a}^{2}+\sigma_{b}^{2}\right)-\left(\sigma_{b}^{2}+4 \sigma_{a}^{2}\right)
$$

3.7.3.7. Esperanca matematica de sopar

A soma de quadrados de parcelas (SQPar) é dada

por SQPar $=\sum_{i=1}^{V} \sum_{j=1}^{b} Y_{i j}^{2}-C_{0}=y P_{\sigma} Y-y P_{1} Y$

Aplicando a esperansa matematica, fica

$E(S Q P a r)=E\left(y \cdot P_{\sigma} y\right)-E\left(y P_{1} y\right)$.

Desenvolvendo-se por partes, tem-se

$E\left(y \cdot P_{\sigma} y\right)=\operatorname{tr}\left(P_{\sigma}^{\Sigma}\right)+\theta^{\prime} X P_{\sigma} X \theta$

$\operatorname{tr}\left(P_{\sigma} \Sigma\right)=\operatorname{tr}\left(P_{\sigma}\right)\left(\sigma_{b}^{2}+u \sigma_{a}^{2}\right)=\frac{1}{u} b k u\left(\sigma_{b}^{2}+u \sigma_{a}^{2}\right)$

$=v r\left(\sigma_{b}^{2}+4 \sigma_{\alpha}^{2}\right)$

$\theta \cdot X \cdot P_{\alpha} X \theta=\left[\mu x_{1}+\beta X_{2}+\tau \cdot x_{3}\right] P_{\sigma}\left[x_{1} \mu+X_{2} \beta+X_{3} \tau\right]$

$=n \mu^{2}+\beta K \beta+2 \beta^{\prime} N^{\prime} \tau+\tau R \tau$

então,

$$
\begin{aligned}
E\left(y_{\sigma}^{\prime} P_{\sigma}\right)= & \mu^{2}+\beta^{\prime} K \beta+2 \beta N \tau+\tau R \tau+v r\left(\alpha_{b}^{2}+u \sigma_{a}^{2}\right) \\
& \text { Desse modo, de }(b .33) \text { e }(b \cdot 11), \text { tem-se } \\
E(S Q P a r)= & \beta^{\prime} K \beta+2 \beta^{\prime} N^{\prime} \tau+\tau R \tau+(v r-1)\left(\alpha_{b}^{2}+u \sigma_{\alpha}^{2}\right)
\end{aligned}
$$

3.7.3.8. Esperança matematica de SQR(a)

A soma de quadrados de residuo a nivel de parcelas, ou simplesmente ressduo(a), representada por SQR (a), $\Leftrightarrow$ obtida por

SQR (a) = SQPar - SQT (aj) - SQEB 
Aplicando a esperança matemática, tem-se $E(\operatorname{SQR}(a))=E[\operatorname{SQPar}]-E[\operatorname{SQT}(a j)]-E[S Q B]$

De $(b .34),(b .16)$ e $(b .19)$, tem-se que $E(\operatorname{SQR}(a))=\beta^{\prime} K \beta+2 \beta^{\prime} N^{\prime} \tau+\tau^{\prime} R \tau+(v r-1)\left(\sigma_{b}^{2}+4 \sigma_{a}^{2}\right)-$ $-\tau \cdot C \tau-(v-1)\left(\sigma_{b}^{2}+4 \sigma_{\alpha}^{2}\right)-2 \beta \cdot N^{\prime} \tau-\beta \cdot K \beta-$ $-\tau \cdot R \tau+\tau C_{\tau}-(b-1)\left(\sigma_{b}^{2}+4 \sigma_{\alpha}^{2}\right)$ $=[v r-1-(v-1)-(b-1)]\left(\sigma_{b}^{2}+u \sigma_{a}^{2}\right)$ $=[v(r-1)-(b-1)]\left(\sigma_{b}^{2}+u \sigma_{a}^{2}\right) d u$ $E(\operatorname{SQR}(a))=(v r-v-b+1)\left(\sigma_{b}^{2}+u o_{a}^{2}\right)$

$(0.35)$

3.7.3.9. Esperanca matematica de $\operatorname{SQR}(b)$

A soma de quadrados de residuo a nivel. de subparcelas, representada por SQR(b), é obtida por

$$
\text { SQR }(b)=\text { SQTot - SQPar - SQT - SQI (b.36) }
$$

Assim, aplicando a esperança matemática, fica $E(\operatorname{SQR}(b))=E(S Q T o t)-E(S Q P a r)-E\left(S Q T^{*}\right)-E(S Q I)$

Substituindo as expressöes encontradas em $(b .31),(b .34),(b .24)$ e $(b .29)$, tem-se $E(\operatorname{SQR}(b))=\beta^{\prime} K \beta+2 \beta^{\prime} \tau+\tau R \tau+\tau \cdot U \tau+\delta \cdot L \delta+$

$$
\begin{aligned}
& +\operatorname{vru}\left(\sigma_{b}^{2}+\alpha_{a}^{2}\right)-\left(\alpha_{b}^{2}+u \sigma_{a}^{2}\right)-\beta^{\prime} K \beta-2 \beta N^{\prime} \tau- \\
& -\tau \cdot R \tau-(v r-1)\left(\sigma_{b}^{2}+u \sigma_{\alpha}^{2}\right)-\tau^{*} U \tau^{*}-(u-1) \sigma_{b}^{2}- \\
& -\delta \cdot L \delta-(v-1)(u-1) \sigma_{b}^{2} \\
& E(\operatorname{SQR}(b))=v(u-1)(r-1) \sigma_{b}^{2}
\end{aligned}
$$

3.7.4. Decomposicao da soma de quadrados de blocos De acordo com o tipo de delineamento em blocos incompletos adotado, a SQB pode ser decomposta em: 1ㄷ Caso: SQB = SQRepetiçбes + SQB1ocos/Repetiçて̌s 2으 Caso: SQB = SQGrupos + SQBlocos/Grupos 
Considerando-se apenas o primeiro caso, a matriz $x_{2}$ relativa aos blocos pode ser decomposta em:

$$
x_{2}=\left[x_{2} \mid x_{0}\right]
$$

sendo $x_{7}$ a matriz de incidencia das repetiços, e

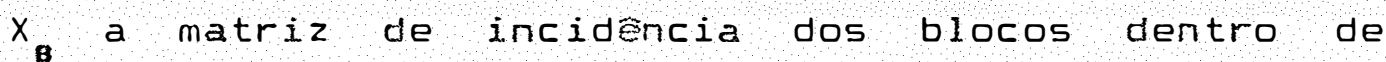
repetiçơs.

Do mesmo modo, o vetor dos efeitos de blocos

fica:

$$
\beta^{\prime}=\left[\rho_{1} \beta_{1}^{\prime}\right]=\left[r_{1} \cdots r_{r} b_{1} / r_{1} \cdots b_{b} / r_{r}\right] .
$$

A soma de quadrados de repetição (SQR) 6 dada

por

$$
S Q R=y^{\prime} x_{7}\left(x_{3} x_{2}\right)^{-1} x_{7} y-\operatorname{COr}=y \cdot P_{7} y-y \cdot P_{1} y
$$

onde: $P_{\gamma}=\frac{1}{V U} I_{\text {(r) }} \otimes E_{\text {(vu) }}$

$$
y \cdot P_{P} y=\frac{1}{v u}\left(y^{2} \cdot 1 \ldots+y^{2} \cdot{ }^{2}\right)=\frac{1}{v u} \sum_{j=1}^{r} R_{j}
$$

sendo, $R_{j}$ o total das observaçes na j-bsima repetică

De acordo com $(b .9)$, tem-se que

$E\left(y \cdot P_{7} y\right)=\operatorname{tr}\left(P_{7} \Sigma\right)+\theta \cdot x \cdot P_{7} x \theta$

desenvolvendo, obtem-5e

$$
E\left(Y \cdot P_{z} Y\right)=\left(\sigma_{b}^{2}+u \sigma_{a}^{2}\right) r+m \mu^{2}+P_{1}^{\prime}\left(x_{7} x_{7}\right) P_{1}
$$

entãn,

$$
E(S Q R)=\left(\sigma_{b}^{2}+4 \sigma_{a}^{2}\right)(r-1)+p_{1}\left(x_{7} x_{7}\right) p_{1}
$$

A soma de quadrados de blocos dentro de repetiços (SQB/R) é obtida por diferenca, isto $6, \mathrm{SQB} / \mathrm{R}=$ $S Q B$ - SQR, sendo calculada por

$$
\begin{aligned}
& S Q B / R=y \cdot x_{B}\left(x_{B} X_{B}\right)^{-1} x_{B} y-y \cdot x_{7}\left(x_{7} x_{7}\right)^{-1} x_{3} y=y^{\prime} P_{B} y-y^{\prime} P_{7} y \\
& \text { onde, } P_{B}=\frac{1}{k u} I_{\langle r b\rangle} \otimes E_{\langle k u\rangle} e
\end{aligned}
$$

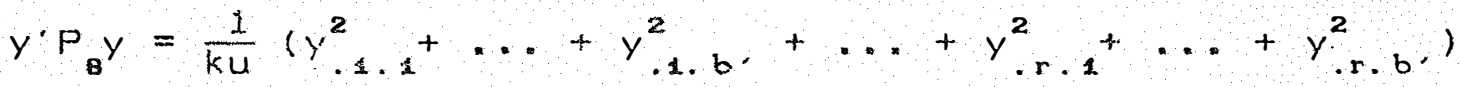

$$
\begin{aligned}
& =\frac{1}{k u} \sum_{j, l}^{b} y^{2} \cdot j \cdot l
\end{aligned}
$$

sendo, $y^{2}$ j.l o total das observaços do l-esimo bloco dentro da j-ésima repeticăo.

De acordo con $(b .9)$, tem-se que

$t\left(Y P_{B} y\right)=\operatorname{tr}\left(P_{B}{ }^{2}\right)+t x P_{B} x \theta$ 
desenvolvendo, obtém-se

$$
\begin{aligned}
E\left(y \cdot P_{\theta} y\right)= & \left(\sigma_{b}^{2}+u \sigma_{\alpha}^{2}\right) r b+n \mu^{2}+\rho_{1}^{\prime}\left(x_{\theta} x_{B}\right) p_{1}+\beta_{1}^{\prime}\left(x_{B} x_{B}\right) \beta_{1}+ \\
& +2 \beta_{1} N^{\prime} \tau+\tau N\left(x_{B} x_{B}\right)^{-1} N \tau
\end{aligned}
$$

logo,

$$
\begin{aligned}
E(S Q B / R)= & \left(\sigma_{b}^{2}+u \sigma_{a}^{2}\right)\left(r b^{\prime}-r\right)+\beta_{1}\left(X_{B} X_{B}\right) \beta_{1}+2 \beta_{1} N \tau+ \\
& +\tau N\left(X_{B} X_{B}\right)^{-1} N \tau
\end{aligned}
$$

onde b'é o número de blocos dentro de cada repetiça.

\subsection{Distribuicao e independencia das formas quadraticas}

Para verificar a distribuiçăo e independência das formas quadraticas apresentadas em (3.7), reportou-se a HALPERIN(1951), MEELROY (1967) e SCARIANO \& DAVENPORT (1987), aplicando-se o seguinte teorema citado em GRAYBILL (1961):

Teorema 1 - Seja y uma vetor multinormal de dimensao $(n) x$ 1), tal que y $\cap N(x \theta, v)$, onde $x \theta$ e vetor de medias e $v e$ a matriz de variâncias e covariancias da distribuição multinormal e sejam $A_{i}(i=1, \ldots, p)$ matrizes simstricas, de posto $k_{i}=r\left(A_{i}\right)$ e $A=\sum_{i=1}^{p} A_{i}$ simétrica, de posto k. Então as formas quadraticas y $A_{i}$ y têm distribuiç̃o $x^{2}$ con $k_{i}$ graus de $1 i b e r-$ liberdade com parámetro de não centralidade $\lambda_{i}=\theta^{\prime} \times A_{i} \times \theta / 2$, sa independentes duas a duas, e ainda, y Ay tem distribuifa $x^{2}$ com k graus de liberdade e parametro de não centralidade $\lambda=\theta^{\prime} \times X^{\prime} A X \theta / 2$, se e somente se ocorrer:

(I) quaisquer duas alternativas são verdadeiras:

(a) $A_{i} V$ é idempotente, para todo $i=1, \ldots, p$,

(b) $A_{i} \vee A_{j}=0$ para todo $i<j$,

(c) AV é idempotente;

ou (II) (c) éverdadeira e (d) $k=\sum_{i=1}^{p} k_{i}$

ou (III) (c) e verdadeira e (e) $A_{1} V, \ldots, A_{p-1} \vee 5 \tilde{o}$ idempotentes e $A_{p} V$ e definida não negativa.

Sejam as matrizes de variâncias e covariâncias dadas por $a^{2} v$, onde $V \in$ tal que: $V E \preccurlyeq=\left\{V \cdot V=c_{1} P_{1}+c_{2} P_{2}+\ldots+c_{p} p_{p}\right.$, para $\left.c_{i}>0\right\} \quad(0.44)$ 
sendo $P_{i}$ tal que, $Y P_{i} Y$ săo formas quadráticas que fornecem as somas de quadrados de interesse para a análise da variância, com $P_{i}(i=1, \ldots, p)$ matrizes de projetores ortogonais $\left(P_{i} P_{j}=0\right.$ se $\left.i \neq k j\right)$, simetricas e idempotentes $\left(P_{i}=P_{i}\right.$ e $\left.P_{i} P_{i}=P_{i}\right)$.

Sob normalidade dos erros, o vetor de erros tendo distribuiçăo multinormal $N\left(\phi, \alpha^{2} v\right)$, onde $v \in \mathfrak{F} e \alpha^{2} e$ uma constante positiva, entäo as condiçes necessarias e suficientes do Teorema 1 aplicadas a $P_{i} / C_{i} \sigma^{2} \quad(i=1, \ldots, p)$ garante que:

$$
\left(y^{\prime} P_{i} y / c_{i} c^{2}\right) \cap x^{2}\left[r\left(P_{i}\right), \lambda_{i}\right],
$$

$e, y \cdot P_{i} Y / c_{i} o^{2}$, são mutualmente independentes,

onde $\lambda_{i}=E\left(y^{\prime}\right) P_{i} E(y) / 2 c_{i} \sigma^{2} e$ o parametro de $n$ ão centralidade. Em consequência, a familia de variáveis aleatorias

$F_{i j}\left(c_{i}, c_{j}\right)=\frac{c_{j}}{r_{i}} \frac{y^{\prime} P_{i} y / r\left(P_{i}\right)}{y P_{j} y / r\left(P_{j}\right)} \cap F\left[r\left(P_{i}\right), r\left(P_{j}\right), \lambda_{i}, \lambda_{j}\right]$ (b.46)

para $V \in \mathcal{F}^{*}$ e $i \neq f_{j}=1, \ldots, p$. Verifica-se que, quando $c_{1}=c_{2}=1$, entzo $F_{12}(1,1)=F$ e a estatIstica "usual" do teste $F$, se ocorrer $V=I$.

As somas de quadrados em termos de formas quadraticas, do modelo de (a.1), obtidas em (3.7), podem ser escritas como

$Y^{\prime} Q_{0} Y=Y^{\prime} Q_{1} Y+Y^{\prime} Q_{2} Y+Y^{\prime} Q_{9} Y+Y^{\prime} Q_{4} Y+Y^{\prime} Q_{5} Y+Y^{\prime} Q_{6} Y+Y^{\prime} Q_{7} Y$

onde $Q_{i}$, com $i=0,1, \ldots, 7$ são os núcleos das formas quadraticas, ou projetores de y sobre algum subespaço de $C(X)$, que fornecem as somas de quadrados de total, correção, blocos, tratamentos principais, residuo(a), tratamentos secundarios, interação e ressduo(b) respectivamente.

Usando o teorema de (b.9), obtiveram-se os seguintes resultados com respeito à distribuição das formas quadráticas:

$$
\begin{aligned}
& Y^{\prime} Q_{1} Y \cap\left(\sigma_{b}^{2}+u \sigma_{b}^{2}\right) x_{\left[1, \lambda_{1}\right]}^{2}, \operatorname{com} \lambda_{1}=n \mu^{2} / 2\left(\sigma_{b}^{2}+4 \sigma_{a}^{2}\right) \\
& Y^{\prime} Q_{2} Y \cap\left(\sigma_{b}^{2}+u \sigma_{b}^{2}\right) x_{\left[b-1, \lambda_{2}\right)^{2}}^{2}
\end{aligned}
$$




$$
\begin{aligned}
& \operatorname{com} \lambda_{2}=\left(2 \beta^{\prime} N^{\prime} \tau+\beta \cdot \beta \beta+\tau K^{-1} N^{\prime} \tau\right) / 2\left(\sigma_{b}^{2}+4 \sigma_{a}^{2}\right) \\
& \left.y^{\prime} Q_{g} y n\left(\sigma_{b}^{2}+u \sigma_{b}^{2}\right) Z_{\left(v-1, \lambda_{9}\right)^{2}}, \operatorname{com} \lambda_{g}=\tau \cdot c^{\prime} \tau\right) / 2\left(\sigma_{b}^{2}+u \sigma_{a}^{2}\right)(0.49) \\
& y^{\prime} Q_{4} Y \cap\left(\alpha_{b}^{2}+u \sigma_{b}^{2}\right) x_{\left(v r-v-b+1, \lambda_{4}\right)^{2}}^{2} \operatorname{com} \lambda_{4}=0 \\
& \left.y^{\prime} Q_{5} y \cap \alpha_{b}^{2} x_{t u-1, \lambda_{5}}^{2}, \operatorname{com} \lambda_{5}=\tau^{*} \cdot u \tau\right) / 2 \sigma_{b}^{2} \\
& Y^{\prime} Q_{\sigma} y \cap \sigma_{b}^{2} x_{\left((v-1)(u-1), \lambda_{\sigma}\right)^{2}}, \operatorname{com} \lambda_{\sigma}=\delta \cdot L \delta / 2 \sigma_{b}^{2} \\
& Y \cdot Q_{7} Y \cap \alpha_{b}^{2} X_{\left(v(u-1)(r-1), \lambda_{7}\right]}^{2}, \operatorname{com} \lambda_{7}=
\end{aligned}
$$

e são independentes entre si. Também de (b.4b), tem-se que a variável aleatória $F_{\mathbf{3 4}}\left(c_{\mathbf{3}}, c_{4}\right)$ dada por

$$
\frac{y^{\cdot} Q_{3} Y /(v-1)}{Y \cdot Q_{4} Y /(v r-v-b+1)} \cap F_{\left[v-1 ; v r-v-b+1 ; \lambda_{9} ; \lambda_{4}\right]}
$$

cu seja,

$$
\frac{\operatorname{SQT}(a j) /(v-1)}{\operatorname{SQR}(a) /(v r-v-b+1)} \cap F_{\left[v-1 ; v r-v-b+1 ; \lambda_{3}\right]}
$$

e, que

$$
F_{57}\left(c_{5}, C_{7}\right)=\frac{y \cdot Q_{5} y /(u-1)}{y \cdot Q_{7} y /[v(u-1)(r-1)]} \cap F_{\left[u-1 ; v(u-1)(r-1) ; \lambda_{5}\right]}
$$

ou seja,

$$
\frac{\operatorname{SQT}^{*} /(u-1)}{\operatorname{SQR}(b) /[v(u-1)(r-1)]} \cap F_{\left[u-1 ; v(u-1)(r-1) ; \lambda_{5}\right]}
$$

e ainda, que

$$
F_{\sigma r}\left(c_{\sigma}, c_{7}\right)=\frac{y^{\prime} Q_{\sigma} Y /(v-1)}{\gamma^{\prime} Q_{7} y /(v r-v-b+1)} \cap F_{\left[(v-1)(u-1) ; v(u-1)(r-1) ; \lambda_{\sigma}\right]}
$$

ou seja,

$$
\frac{\operatorname{SQI} /(v-1)(u-1)}{\operatorname{SQR}(b) /[v(u-1)(r-1)]} \cap F_{\left[(v-1)(u-1) ; v(u-1)(r-1) ; \lambda_{\sigma}\right]}
$$




\subsection{Esquema de analise da variancia}

- esquema de análise da variancia com as respectivas esperangas matemáticas de quadrados medias está apresentado na Tabela 1.

Tabela 1 - Es uema da análise da variáncia com as respectivas esperanças matemática de quadrados medios.

\begin{tabular}{|c|c|c|c|}
\hline $\begin{array}{l}\text { Causas de } \\
\text { Variaça }\end{array}$ & $\begin{array}{l}\text { Graus de } \\
\text { liberdade }\end{array}$ & $\begin{array}{l}\text { Somas de } \\
\text { quadrados }\end{array}$ & $\begin{array}{l}\text { Esperanga dos } \\
\text { quadrados médios }\end{array}$ \\
\hline Media & 1 & $Y^{\circ} Q^{Y}$ & $c_{1}+n \mu^{2}$ \\
\hline Blocos & $b-1$ & $Y^{\circ} Q_{2}^{Y}$ & $c_{2}+\frac{\tau \cdot N K^{-1} N \tau+2 \beta^{\prime} N \tau+\beta^{\prime} K \beta}{b-1}$ \\
\hline $\operatorname{Trat} \cdot P \cdot(\mathrm{aj})$ & $v-1$ & $Y Q_{B} Y$ & $c_{3}+\tau C \tau /(v-1)$ \\
\hline Residuo (a) & $b k-b-v+1$ & $Y Q^{Y}$ & -4 \\
\hline Parcelas & $r v-1$ & $Y P^{\prime} Y$ & - \\
\hline $\operatorname{Trat} . S=c$ & $u-1$ & $\mathrm{Y}^{\prime} \mathrm{Y}$ & $c_{5}+\tau^{*} u \tau /(u-1)$ \\
\hline Interasao & $(v-1)(u-1)$ & $Y^{\circ} Q_{\sigma} Y$ & $c_{\sigma}+\delta L \delta /(v-1)(u-1)$ \\
\hline Residuo(b) & $v(u-1)(r-1)$ & $Y^{\prime} Q_{7}$ & $c_{7}$ \\
\hline
\end{tabular}

onde: $c_{1}=c_{2}=c_{3}=c_{4}=\left(\sigma_{b}^{2}+u \sigma_{a}^{2}\right), c_{5}=c_{\sigma}=c_{3}=\alpha_{b}^{2}$

\subsection{Estimabilidale}

A estimabilidade se fundamenta em funçós estimáveis do tipo $\lambda^{\prime} \theta$ associadas ao modelo linear $y=x \theta+\varepsilon$, sendo $\lambda \cdot \theta$ estimavel se puder ser expressa como una funcão 1 inear das esperanças das observações. São estimáveis as funçbes de tratamento $\lambda \cdot \theta$, tais que, $r[x]=r[x: \lambda]$. Naturalmente, os contrastes são un subconjunto de tais funç̋อs, e por serem de grande importancia prática, serão abordados

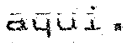


Se $\lambda^{\prime} \theta$ é uma função estimável, então pelo teorema de Gauss-Markov, seu BLUE (Best Linear Unbiased Estimator) e $\operatorname{BLUE}\left(\lambda^{\hat{*}} \theta\right)=\lambda^{\prime} \theta^{\circ}$, para qualquer $\theta^{\circ}$ solução das equaçoses normais, de acordo com IEMMA (1987).

o valor esperado de qualquer observação é uma função estimável, conforme SEARLE (1987); ạssim $E(y)=x \theta$ ou

$$
E\left(y_{i j l}\right)=\mu+b_{j}+t_{i}+t_{i}^{*}+\delta_{i l} \forall i, j, l
$$
constituem uma coleção de funçzes básicas estimáveis, segundo IEMMA(1987). Como exemplo, o melhor estimador linear näo viesado, representado por BLUE, de $\mu+b_{j}+t_{i}+t_{l}^{*}+\delta_{i l}$ $E y_{i j l}$, sendo sua varisincia dada por $\operatorname{var}\left(y_{i j l}\right)=\alpha_{a}^{2}+\alpha_{b}^{2}$, cuja estimativa é

$\hat{\operatorname{Var}}\left(y_{i j l}\right)=[\operatorname{QMR}(a)-\operatorname{QMR}(b)] / u+\operatorname{QMR}(w)=\frac{\operatorname{QMR}(a)+(u-1) \operatorname{QMR}(b)}{u}$ Je modo que QMR(b) é uma estimativa năo viesada de $\sigma_{b}^{2} e$, o QMR (a) e uma estimativa nao viesada de $\alpha_{b}^{2}+u \alpha_{a}^{2}$.

Todas combinaçơes lineares de funçơses estimáveis, são também estimáveis, desde que não haja desconexão, de acordo com RAO (1946), SEARLE (1971) פ IEMMA (1987); pode-se então, obter combinaç̇es lineares de interesse, das observaças. No exemplo de $(3.1)$, a combinação linear das observaçzes

$$
y_{922}-y_{422}=t_{9}-t_{4}+\delta_{92}-\delta_{42}+\delta_{322}-\varepsilon_{422}
$$

pode ser usada para medir o efeito entre os tratamentos principais 3 e 4 dentro do tratamento secundário 2. Assim, o contraste $\lambda_{t}^{\prime} \theta=t_{3}-t_{4}+\delta_{32}-\delta_{42}$, possui BLUE dado por $\lambda_{1}^{\prime} \theta^{\circ}=y_{322}-y_{422}$, ou seja $E\left[\lambda_{1} \theta^{\circ}\right]=E\left[y_{322}-y_{422}\right]=t_{9}-t_{4}+\delta_{32}-\delta_{42}$.

Sua variância é

$$
\begin{aligned}
\operatorname{Var}\left(i \alpha_{1} \theta^{\circ}\right) & =\operatorname{Var}\left(y_{322}-y_{422}\right)=E\left[\eta_{32}+e_{32 \%}-\eta_{42}-e_{422}\right]^{2} \\
& =2\left(\sigma_{\alpha}^{2}+\sigma_{b}^{2}\right)
\end{aligned}
$$

cuja estimativa $s$ 
$\operatorname{var}\left(\lambda \cdot \theta^{\circ}\right)=2 \frac{\operatorname{QMR}(a)+(u-1) \operatorname{QMR}(b)}{u}$

Do mesmo modo, o contraste

$\lambda_{2} \theta=t_{1}-t_{2}+\delta_{11}-\delta_{21}$

$=[000001-100000100-10000000000$,

e uma funcão linear estimável. 0 BLUE de $\lambda_{2} \theta$ e y $111-y_{211}$, cuja variancia e $\operatorname{var}\left(\lambda_{z} \theta^{0}\right)=2\left(\sigma_{a}^{2}+\sigma_{b}^{2}\right)$ e, sua estimativa é $\operatorname{Var}\left(\lambda\left(\theta^{0}\right)=2 \frac{\operatorname{QMR}(a)+(u-1) \operatorname{QMR}(b)}{u}\right.$

- BLUE da função linear estimável

$$
\lambda_{9} \theta^{0}=t_{1}^{*}-t_{2}^{*}+\delta_{11}-\delta_{12}, \Leftrightarrow \bar{y}_{1,1}-\bar{y}_{1.2}
$$

cuja variancia e $\operatorname{Var}\left(\lambda_{3} \theta^{0}\right)=\frac{2}{r} \sigma_{b}^{2}$ e sendo sua estimativa

$$
\operatorname{Var}\left(\bar{y}_{1.1}-\bar{y}_{1.2}\right)=\frac{2}{r} \operatorname{QMR}(b) .
$$

No caso de duas medias de tratamentos prine:pais en um mesmo tratamento secundario, para o exemplo ilustrativo, tem-se que

$$
E\left(\bar{y}_{1,1}-\bar{y}_{3,1}\right)=\left(b_{1}-b_{2}\right) / 2+t_{1}-t_{3}+\delta_{11}-\delta_{31} \text {, }
$$

portanto, $\bar{y}_{1.1}-\bar{y}_{9.1}$ não e um estimador não-viesado da diferença entre os tratamentos frincipais 1 e 3 no tratamento secundario 1, pois inclui uma diferenca entre blocos. Assim, por causa da presença de blocos, diferenças entre medias de tratamentos principais num mesmo tratamento secundário não são estimáveis.

No tocante às funçơes estimáveis de tratamentos principais pode-se verificar que uma funçăo Iinear paramétrica do tipo $\lambda^{\prime} \tau$ e estimável no modelo

$$
y=x_{1} \mu+x_{2} \beta+x_{3} \tau+x_{4} \tau^{*}+x_{5} \delta+\varepsilon
$$

se, e somente se, $\lambda^{\prime} \in \subset\left\{X_{3}^{\prime}\left(I-P_{2}\right) X_{3}\right\}$ ou $\lambda^{\prime} \in c(C)$.

observa-se que a matriz c tem dimensbes menores do que $x \cdot X$, facilitando a obtenção de soluções e, nesse caso, uma função linear paramétrica é estimável, somente se $r[C]=r[C(\lambda]$, ou seja, $\lambda$, ou $\lambda$ deve pertencer ao espaco coluna de $C$, pois $C$ é uma matriz simetrica.

Por exemplo. as funfoes lineares $t_{i}-t_{i}$ săo 
80.

estimáveis, enquanto que $t_{i} \Leftrightarrow$ não estimável. Assim, para o exemplo ilustrativo de $(3.1)$, aplicando operaçes elementares nas linhas de $[C: \cdot \lambda]$, obtem-se

$$
\left[\begin{array}{cccc:cc}
2 & -1 & -1 & 0 & 1 & 1 \\
-1 & 2 & 0 & -1 & -1 & 0 \\
-1 & 0 & 2 & -1 & 0 & 0 \\
0 & -1 & -1 & 2 & 0 & 0
\end{array}\right] \approx\left[\begin{array}{cccc:cc}
1 & -1 & 0 & 0 & 3 / 4 & 0 \\
0 & 1 & -1 & 0 & -1 / 2 & 0 \\
0 & 0 & 1 & -1 & 1 / 4 & 0 \\
0 & 0 & 0 & 0 & 0 & 1
\end{array}\right]
$$

Logo, a função $t_{1}-t_{2}$ é estimável, e a função $t_{1}$ é não estimável. De modo geral, $t_{i}-t_{i}$ constituem um conjunto de funçöes estimaveis, conhecidos como contrastes elementares, para o modelo irrestrito.

Se: $\lambda^{\prime} \tau$ é estimável no modelo

$$
y=x_{1} \mu+x_{2} \beta+x_{3} \tau+x_{4} \tau^{*}+x_{5} \delta+\varepsilon
$$

entã o seu BLUE pode ser obtido por $\lambda^{\prime} \tau^{\circ}$, que $\Leftrightarrow$ invariante para qualquer $\tau^{\circ}$ solução do SENR.

Seja $\lambda^{\prime} \tau$ uma função linear estinável, então a variância de $\lambda \cdot \hat{\tau}$ édada por

$$
\begin{aligned}
\operatorname{Var}\left(\lambda^{\prime} \tau\right)= & E\left[\left[\lambda^{\prime} \hat{\tau}-E\left(\lambda^{\prime} \hat{\tau}\right)\right]\left[\lambda^{\prime} \hat{\tau}-E\left(\lambda^{\prime} \hat{\tau}\right)\right]^{\prime}\right\} \\
= & E\left[\lambda^{\prime}[\hat{\tau}-E(\hat{\tau})][\hat{\tau}-E(\hat{\tau})] \cdot \lambda\right] \\
= & \lambda^{\prime} E\left[[\hat{\tau}-E(\hat{\tau})][\hat{\tau}-E(\hat{\tau})]^{\prime} \lambda\right. \\
= & \lambda^{\prime} D(\hat{\tau}) \lambda^{\prime} \\
& \operatorname{Var}\left(\lambda^{\prime} \tau\right)=\lambda^{\prime} M^{-1} C M^{-1} \lambda\left(\sigma_{b}^{2}+4 \sigma_{\alpha}^{2}\right)
\end{aligned}
$$

Da definiçă de estimabilidade (RAO, 1946), tem-se que, se $\lambda^{\prime} \tau$ e uma funç̃o estimável, então existe pelo menos um vetor $a^{\prime} \not 2 \varnothing$, tal que $\lambda^{\prime}=a^{\prime} C \Rightarrow \lambda=$ Ca. Assim, substituindo em $\left(b_{*} 58\right)$, fica

$$
\begin{aligned}
& \operatorname{Var}(\lambda \cdot \hat{\tau})=\lambda \cdot M^{-1} C M^{-1} \operatorname{Ca}\left(\sigma_{b}^{2}+4 \alpha_{a}^{2}\right) \\
& \text { Mas, segundo OGAWA (1974) e IEMMA (1987), a }
\end{aligned}
$$

ma- $\operatorname{triz} M^{-1}$ é uma inversa condicional de $C$, com a propriedade $C M^{-1} C=C M^{-1} C=C$, pois $C$ uma matriz simétrica. Logo,

$$
\operatorname{Var}(\lambda \cdot \hat{\tau})=\lambda \cdot M^{-1} \operatorname{Ca}\left(\sigma_{b}^{2}+u \sigma_{a}^{2}\right)
$$

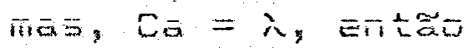




$$
\operatorname{var}(\lambda \cdot \hat{\tau})=\lambda \cdot M^{-1} \lambda\left(\sigma_{b}^{2}+u \sigma_{a}^{2}\right)
$$

cuja estimativa

$$
\hat{\operatorname{Var}}(\lambda \cdot \hat{\tau})=\lambda \cdot M^{-1} \lambda \operatorname{QMR}(a)
$$

Assim, no caso particular de comparação entre dois efeitos estimados de tratamentos principais, tem-se que $\lambda \cdot \hat{\tau}=\hat{t}_{i}-\hat{t}_{i}$ cuja estimativa da variancia

$$
\operatorname{Var}\left(\lambda^{\prime} \hat{\tau}\right)=\operatorname{Var}\left(\hat{t}_{i}-\hat{t}_{i}\right)=\left(m_{i i}+m_{i} \cdot-m_{i i}-m_{i}\right) \operatorname{QMR}(a)
$$

onde os elementos $\left(m_{i i}\right)$ da matriz $M^{-1}$ estão definidos em $(a .41)$ ou $(a .57)$ ou $(a .60)$ ou $(a .71)$.

\section{Sendo $\lambda^{\prime} \tau^{*}$ uma funç̃a paramétrica estimável,} com BLUE dado for $\widehat{\lambda}_{\tau}^{*}$, então

$$
\operatorname{Var}\left(\widehat{\lambda} \cdot \tau^{*}\right)=\lambda \cdot \operatorname{Var}\left(\hat{\tau}^{*}\right) \lambda=\lambda \cdot D\left(\hat{\tau}^{*}\right) \lambda
$$

com $D\left(\hat{\tau}^{*}\right)$ sendo a matriz de dispersão dos efeitos de tratamentos secundários de $(0.90)$.

No caso particular de um contraste entre efeitos de dois tratamentos secundários, tem-se $\lambda^{\prime} \hat{\tau}^{*}=\hat{t}_{l}^{*}-\hat{t}_{\imath}^{*}$.

Se $\lambda \mathcal{S}$ é uma função paramétrica estimável, com BLUE dado por $\widehat{\lambda} \delta$, então

$$
\operatorname{var}(\hat{\lambda} \delta)=\lambda \cdot D(\hat{\delta}) \lambda
$$

com $D(\hat{\delta})$, a matriz de dispersão dos efeitos da interação, de $(a .96)$.

Para o caso particular de um contraste elementar, têm-se os sequintes casos de interesse:

(i) $\hat{\delta}_{i l}-\hat{\delta}_{i l}$ diferença entre os efeitos estimados de dois tratamentos secundários, em um mesmo tratamento principal; (ii) $\hat{\delta}_{i l}-\hat{\delta}_{i, l}$ diferença entre os efeitos estimados de dois tratamentos principais, em um mesmo tratamento secundário.

$$
0 \text { BLUE de } t_{i}^{*}-t_{i}^{*}+\delta_{i l}-\delta_{i i} \text { e } \bar{y}_{i, l}-\bar{y}_{i, l} \text {. }
$$

cuja variância $\in \operatorname{Var}\left(\bar{y}_{i, l}-\bar{y}_{i . l}\right)=\frac{2}{r} \alpha_{b}^{2}$. Este contraste mede a diferenca entre duas medias de tratamentos secundários num mesmo tratamento principal.

Para dois tratamentos principais em um mesmo tratamento secundario pode-se usar combinaçoses lineares eslimáveis das observacoes, por exempio, o DLUE ví 
82.

$$
t_{1}-t_{2}+\delta_{11}-\delta_{21} \& y_{111}-y_{211}
$$

cuja variancia $\Leftrightarrow \operatorname{Var}\left(y_{111}-y_{211}\right)=2\left(\sigma_{a}^{2}+\sigma_{b}^{2}\right)$, e sua estimativa é $\hat{\operatorname{Var}}\left(\gamma_{111}-y_{211}\right)=2[(u-1) \operatorname{QMR}(b)+\operatorname{QMR}(A)] / u$.

Do mesmo modo, o BLUE de

$$
t_{3}-t_{4}+\delta_{32}-\delta_{42} \& y_{322}-y_{422}
$$

cuja variância é $\operatorname{Var}\left(y_{322}-y_{422}\right)=2\left(\sigma_{a}^{2}+\sigma_{b}^{2}\right)$. 0 qual serve para verificar a diferença entre as medias dos tratamentos principais 3 e 4 dentro do tratamento secundário 2.

\subsection{Testes de hipóteses}

Nos ensaios em parcelas subdivididas, em geral, existe interesse em testar as seguintes hipóteses bésicas:

$$
\begin{aligned}
& H_{0}^{1}: t_{i}=0, \forall i=1,2, \ldots, v \\
& H_{0}^{2}: t_{i}=0, \forall 1=1,2, \ldots, u \\
& H_{0}^{3}: \mathcal{E}_{i l}=0, \forall i=1,2, \ldots, v ; 1=1,2, \ldots, 4
\end{aligned}
$$

que correspondem às hipóteses de não existencia de efeitos de tratamentos principais, de tratamentos secundarios $e$ de interaçăo respectivamente, dé acordo com IEMMA (1983).

Os erros apropriados para se verificiar essas hipóteses, săo evidentes quando se observam as esperanças matemáticas dos quadrados medios, apresentadas na Tabela 1 , visto que, as esperanças do numerador e denominador das estatisticas F, devem diferir somente na função positiva do efeito fixo que se deseja testar.

No experimento em parcela subdividida, quando a hipstese $H^{3}$, referente a interaça $\left(T \times T^{*}\right)$ e rejeitada, $\epsilon$ um indicio de que os tratameritos secundários se comportam dife- rentemente em relação aos tratamentos principais. Assim, é conveniente que seja estudado o comportamento dos $T^{*}$ dentro de cada $T$ ou $T$ dentro de $T^{*}$, cujas hipóteses a serem testadas $5 \widetilde{0}$ :

$$
\begin{aligned}
& \text { (i) } H_{0}^{1}: t_{i\langle l}=0, \forall i=1,2, \ldots, v \\
& \text { iij) } H_{0}^{5}=t_{(i)}=0, \forall i=1,2, \ldots, i
\end{aligned}
$$


83.

Ds criterios para essas duas hipóteses estão apresentados nas Tabelas $2 a$ e 2 b, dadas a sequir.

Tabela $2 a$ - Esquema da decomposição da soma dos graus de 1 iberdade da $5 Q$ interação tratamentos principais com tratamentos secundários com os graus de 1 berdade da $S Q$ tratamentos secundários.

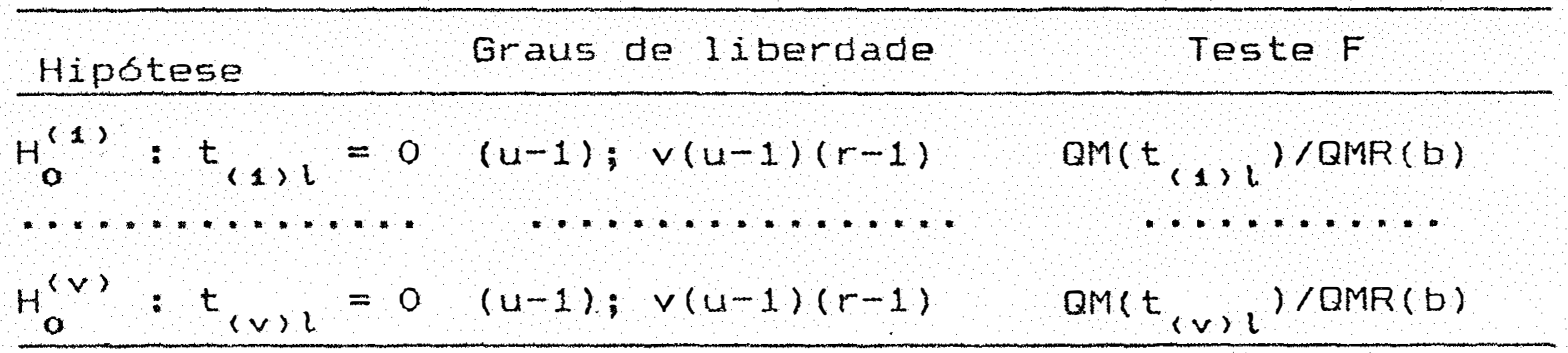

onde: $5 Q t, i) l=\left(\sum_{l=1}^{u} y_{i, l}^{2}\right) / r \quad-\left(y_{i}^{2}, \ldots\right) / r u$ para $i=1,2, \ldots, v$ $\frac{[S Q(t, u)] /(u-1)}{\operatorname{SQR}(b) / v(u-1)(r-1)} \cap F[u-1 ; v(u-1)(r-1) ; \lambda, i, l]$

$\operatorname{com} \lambda_{l i) l}=\left(r \sum_{l=1}^{u} t_{l}^{2}+r \sum_{l=l}^{u} \delta_{i l}^{2}\right) / 2 \sigma_{b}^{2}$ 
Tabela 2b - Esquema da decomposição da soma dos graus de liberdade da $S Q$ interação dos tratamentos principais com tratamentos secundarios com graus de liberdade da $S Q$ tratamentos principais

Hipotese Graus de liberdade Teste F

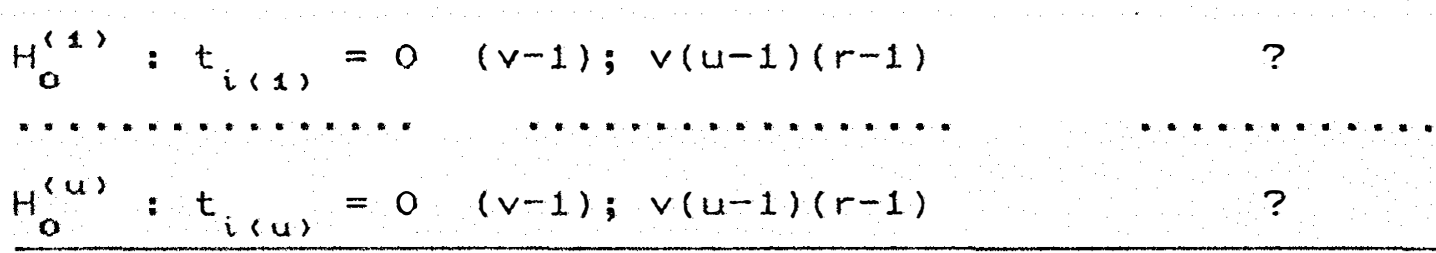

onde: $S Q t_{i(l)}=\left(\sum_{i=1}^{v} y_{i, l}^{2}\right) / r-\left(y^{2}, l\right) / r v \operatorname{para} l=1,2, \ldots, u$ $\frac{\left[\operatorname{SQ}\left(t_{i(l,}\right)\right] /(v-1)}{\operatorname{SQR}(b) / v(u-1)(r-1)} \cap F_{\left[v-1 ; v(u-1)(r-1) ; \lambda_{i(l)}\right]}$

$\operatorname{com} \lambda_{i(l)}=\left(r \sum_{i=1}^{v} t_{i}^{2}+r \sum_{i=1}^{v} \delta_{i l}^{2}+\sum_{j=1}^{b} b_{j}^{2}\right) / 2\left(\sigma_{a}^{2}+\alpha_{b}^{2}\right)$

Para essas u hipoteses, nåo existe um teste $F$ exato ou apropriado, visto que, no parametro de não centralidade $\lambda_{i l l}$ existe uma funçăo positiva dos efeitos de blocos, isto $6, n \approx 0$ se esta testando apenas o efeito de tratamentos primários dentro de lim tratamento secundário, mas tambtm, um efeito de blocos, conforme pode-se verificar pela expressão de parămetro de não centralidade $\lambda_{i(l)}$, apresentado na Tabela $2 b$.

\subsection{Comparaçoes mútiplas}

Admitindo-se a possibilidade de rejeiça das hipóteses de nulidade, referentes aos efeitos de tratamentos principais e secundários e da interaçă, para contimuar a análise estatistica, deve- se considerar os seguintes casos de comparaç̋es múltiplas, dados a seguir. 


\subsubsection{Um contraste entre duas medias de trata- mentos principais}

Reportando-se a (a.41), tem-se que, no caso de um contraste entre duas médias de tratamentos principais, podem ser considerades as seguintes tipos de contrastes entre médias de dois tratamentos principais:

a) Dois tratamentos principais que são i-6simos associados

$$
\begin{aligned}
\operatorname{var}\left(\hat{m}_{i}-\hat{m}_{i^{\prime}}\right) & =\left(m_{i i}+m_{i \cdot i}-m_{i i}-m_{i-i}\right)\left(\sigma_{b}^{2}+u \sigma_{a}^{2}\right) \\
& =\left[\frac{2}{u r(k-1)}-\frac{2}{r(k-1)}\right] \sum_{l=1}^{m} \lambda_{l} f_{l i}\left(\sigma_{b}^{2}+u \sigma_{a}^{2}\right) \\
& \operatorname{var}\left(\hat{m}_{i}-\hat{m}_{i^{\prime}}\right)=\frac{2}{u r(k-1)}\left[k-u \sum_{l=1}^{m} \lambda_{l} f f_{l i}\right]\left(\sigma_{b}^{2}+u \sigma_{a}^{2}\right)
\end{aligned}
$$

no caso geral de PBIB(m), e cuja estimativa é

$$
\begin{gathered}
\operatorname{Var}\left(\hat{m}_{i}-\hat{m}_{i},\right)=\frac{2}{u r(k-1)}\left(k-u \sum_{l=1}^{m} \lambda_{l} f_{l i}\right) \operatorname{QMR}(a) \\
\text { No caso de PBIB(2), tem-se }
\end{gathered}
$$

b.1) Dis tratamentos principais que săo primeiros associados

$$
\operatorname{var}\left(\hat{m}_{i}-\hat{m}_{i}\right)=2 \frac{k \Delta-u A_{1}}{u r(k-1) \Delta}\left(\sigma_{b}^{2}+4 \sigma_{a}^{2}\right)
$$

b.2) Dois tratamentos principais que sao sequndos associados

$$
\operatorname{var}\left(\hat{m}_{i}-\hat{m}_{i}\right)=2 \frac{k \Delta-u A_{2}}{u r(k-1) \Delta}\left(\sigma_{b}^{2}+u \sigma_{\alpha}^{2}\right)
$$

b.3) Uma varianncia media

$$
\begin{aligned}
& \operatorname{var}\left(\hat{m}_{i}-\hat{m}_{i},\right)=2 \frac{k(v-1) \Delta-u\left(n_{1} A_{1}+n_{2} A_{2}\right)}{u r(k-1)(v-1) \Delta}\left(\sigma_{b}^{2}+u \sigma_{a}^{2}\right) \\
& \text { No caso de reticulados quadrados, tem-se }
\end{aligned}
$$

c.1) Dois tratamentos principais que são primeiros associados

$$
\operatorname{var}\left(\hat{m}_{i}-\hat{m}_{i}\right)=\frac{2(k+1)}{u r k}\left(\sigma_{b}^{2}+u \sigma_{a}^{2}\right)
$$

c.2) Dois tratamentos principais que são segundos associados

$$
\operatorname{var}\left(\hat{m}_{i}-\hat{m}_{i},\right)=\frac{2}{u r}\left[1+\frac{r}{k(r-1)}\right]\left(\sigma_{b}^{2}+u \alpha_{a}^{2}\right)
$$

c.3) Una variáncia média es 


$$
\begin{aligned}
& \operatorname{Var}\left(\hat{m}_{i}-\hat{m}_{i}\right)=\frac{2}{u r}\left[1+\frac{r}{(k+1)(r-1)}\right]\left(\sigma_{b}^{2}+u \sigma_{a}^{2}\right) \quad(0.71) \\
& \text { No caso especial onde } \lambda_{1}=\lambda_{2}=\ldots=\lambda_{v}=\lambda,
\end{aligned}
$$
as variancias anteriores se, modificam para:

$$
\operatorname{var}\left(\hat{m}_{i}-\hat{m}_{i}\right)=\frac{2 k}{\lambda v u}\left(\sigma_{b}^{2}+u \sigma_{a}^{2}\right)
$$

cuja estimativa 6

$$
\operatorname{Var}\left(\hat{m}_{i}-\hat{m}_{i}\right)=\frac{2 k}{\lambda v u} \operatorname{QMR}(a)
$$

a qual concorda com o resultado obtido por IEMMA (1981), para os experimentos em blocos incompletos balanceados.

\subsubsection{Um contraste entre duas medias de tratamen- tos secundartos \\ Se $\lambda \cdot \tau^{*}$ e uma função paramétrica estimável que} envolve efeitos de dois tratamentos secundários, então reportando-se a $(a .14)$, tem-se que:

$$
\operatorname{var}\left(\hat{t}_{l}^{*}-\hat{t}_{l}^{*}\right)=\operatorname{var}\left(\hat{m}_{l}-\hat{m}_{l}\right)=\frac{2}{\operatorname{vr}} \sigma_{b}^{2}
$$

- qual $\Leftrightarrow$ um resultado identico aqueles encontrados na literatura para a variancia entre duas médias de tratamentos secundários obtidas de experimentos em parcelas subdivididas em blocos incompletos balanceados (IEMMA, 1981). Cuja estimativa não viesada

$$
\operatorname{Var}\left(\hat{m}_{l}-\hat{m}_{l}\right)=\frac{2}{\operatorname{Vr}} \operatorname{QMR}(b)
$$

sendo uma estimativa não tendenciosa para $\alpha_{b}^{2}$, o quadrado médio do residuo(b).

3.12.3. Um contraste entre duas medias de tratamentos secundarios, dado um tratamento principal

A variăncia para a diferença entre duas medias de tratamentos secundários dentro de um mesmo tratamento principal, foi obtida a partir das médias do exemplo ilustrativo de (3.1). Assim 


$$
\begin{gathered}
\bar{y}_{1.1}=\mu+\frac{b_{1}+b_{3}}{r}+t_{1}+t_{1}^{*}+\delta_{11}+\frac{\eta_{11}+\eta_{13}}{r}+\frac{e_{111}+e_{131}}{r} \\
\bar{y}_{1.2}=\mu+\frac{b_{1}+b_{3}}{r}+t_{1}+t_{2}^{*}+\delta_{12}+\frac{\eta_{11}+\eta_{19}}{r}+\frac{e_{112}+e_{132}}{r} \\
\text { Generalizando, tem-5e }
\end{gathered}
$$$$
\bar{y}_{i, l}=\mu+\phi b(i)+t_{i}+t_{i}^{*}+\delta_{i l}+\frac{\eta_{i}}{r}+\frac{e_{i \cdot l}}{r}
$$$$
\bar{y}_{i, l^{\circ}}=\mu+\phi b(i)+t_{i}+t_{i}^{*}+\delta_{i l}+\frac{n_{i \cdot}}{r}+\frac{e_{i \cdot l}}{r}
$$

sendo $\phi b(i)$ a soma dos efeitos dos blocos que contem o i-ésimo tratamento primário

$$
\text { Usando a definição de variancia, tem-se }
$$

$$
\begin{aligned}
& \operatorname{Var}\left[\bar{y}_{i, l}-\bar{y}_{i, l,}\right]=E\left[\bar{y}_{i, l}-\bar{y}_{i, l}-E\left(\bar{y}_{i, l}-\bar{y}_{i, l}\right)\right]^{2} \\
& \operatorname{mas}, \quad E\left(\bar{y}_{i, l}-\bar{y}_{i, l}\right)=t_{l}-t_{l}^{*}+\delta_{i l}-\delta_{i l}
\end{aligned}
$$

entao

$$
\begin{aligned}
\operatorname{Var}\left(\bar{y}_{i, l}-\bar{y}_{i, l}\right)= & E\left[\frac{e_{i . l}}{r}-\frac{e_{i \cdot l}}{r}\right]^{2} \\
& =\frac{1}{r^{2}} E\left[e_{i, l}^{2}+e_{i, l}^{2}-2 e_{i, l} e_{i, l}\right] \\
& =\frac{1}{r^{2}}\left[r \sigma_{b}^{2}+r \sigma_{b}^{2}-0\right] .
\end{aligned}
$$

$\operatorname{Var}\left(\bar{y}_{i, l}-\bar{y}_{i, l},\right)=\frac{2}{r} \sigma_{b}^{2}$

cuja estimativa para a diferenca entre duas medias de tratamentos secundários em um mesmo tratamento principal ficou

$$
\operatorname{Var}\left(\bar{y}_{i . l}-\bar{y}_{i . l} .\right)=\frac{2}{r} \operatorname{QMR}(b)
$$

resultado análogo àqueles encontrados na literatura para os experimentos em parcelas subdivididas.

3.12.4. Um contraste entre duas medias de tratamentos principais, dado un tratamento secundario

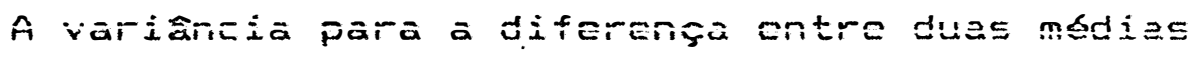


88.

de tratamentos principais dentro de um mesmo tratamento secundário, foi obtida a partir das médias do exemplo ilustrativo de (3.1). Assim sendo, as medias são dadas por

$$
\begin{aligned}
& \bar{y}_{1.1}=\mu+\frac{b_{1}+b_{9}}{r}+t_{1}+t_{1}^{*}+\delta_{11}+\frac{\eta_{11}+n_{13}}{r}+\frac{e_{111}+e_{191}}{r} \\
& \bar{y}_{9.1}=\mu+\frac{b_{2}+b_{9}}{r}+t_{9}+t_{1}+\delta_{91}+\frac{n_{92}+n_{93}}{r}+\frac{e_{921}+e_{931}}{r}
\end{aligned}
$$

Generalizando, tem-se

$$
\begin{aligned}
& \bar{y}_{i, l}=\mu+\phi b(i)+t_{i}+t_{l}^{*}+\delta_{i l}+\frac{\eta_{i}}{r}+\frac{e_{i \cdot l}}{r} \\
& \bar{y}_{i \cdot l l}=\mu+\phi b(i)+t_{i}+t_{l}^{*}+\delta_{i \cdot l}+\frac{\eta_{i \cdot}}{r}+\frac{e_{i \cdot l l}}{r}
\end{aligned}
$$

Aplicando a definiçăo de variancia, tem-se

$$
\operatorname{var}\left[\bar{y}_{i, l}-\bar{y}_{i^{\prime}, l}\right]=E\left[\bar{y}_{i, l}-\bar{y}_{i^{\prime}, l}-E\left(\bar{y}_{i, l}-\bar{y}_{i^{\prime}, l}\right)\right]^{2}
$$

Do mesmo modo anterior, tem-se

$$
\begin{aligned}
\operatorname{Var}\left(\bar{y}_{i, l}-\bar{y}_{i \cdot l}\right)= & E\left[\frac{\eta_{i} \cdot}{r}-\frac{\eta_{i \cdot}}{r}+\frac{\theta_{i \cdot l}}{r}-\frac{\theta_{i \cdot l}}{r}\right]^{2} \\
& =\frac{1}{r^{2}}\left[r \alpha_{a}^{2}+r \sigma_{a}^{2}+r \sigma_{b}^{2}+r \sigma_{b}^{2}\right]
\end{aligned}
$$

$\operatorname{Var}\left(\bar{y}_{i, l}-\bar{y}_{i \cdot, l}\right)=\frac{2}{r}\left[\sigma_{a}^{2}+\sigma_{b}^{2}\right]$

cuja estimativa para a diferenca entre duas medias de tratamentos principais em um mesmo tratamento secundário foi

$$
\operatorname{var}\left(\bar{y}_{i, l}-\bar{y}_{i \cdot . l}\right)=\frac{2}{r} \frac{\operatorname{QMR}(a)+(u-1) \operatorname{QMR}(b)}{u}
$$

resultado análogo aquele encontrado por PIMENTEL. GOMES (1985), que é uma combinação linear entre os residuo (a) e (b), quando o experimento foi instaladoem parcelas subdivididas.

3.12.5. Um contraste entre dois efeitos estimados de tratamentos secundarios, dado um tratamento principal

Tomando-se o contraste $\delta_{i l}-\delta_{i l}$, cujo BLUE $\varepsilon$ $\bar{y}_{i . l}-\bar{y}_{i . l}-\left(\bar{y}_{. . l}-\bar{y}_{. l}\right\}=$ e reportando-se a $(3.6 .6)$, 
tem-se que

$$
\operatorname{var}\left(\hat{\delta}_{i l}-\hat{\delta}_{i l}\right)=\frac{2(v-1)}{v r} \sigma_{b}^{2}=\frac{2}{r} \frac{v-1}{v} \sigma_{b}^{2}
$$

resultado analogo aquele encontrado por CHAKRABARTI (1962), quando o experimento com parcelas subdivididas foi instalado em blocos casualizados, e por IEMMA (1981), para os experimentos em blocos incompletos balanceados.

\subsubsection{Um contraste entre dois efeltos estimados de tratamentos principals, dado um trata- mento secundario}

Tomando-se o contraste $\delta_{i l}-\delta_{i-1}$, cujo BLUE e $\bar{y}_{i, l}-\bar{y}_{i, l}-\left(\bar{y}_{i .}-\bar{y}_{i, \ldots}\right)$, e reportando-5e a $(3.6 .6)$, tem-se que

$$
\operatorname{var}\left(\hat{\sigma}_{i l}-\hat{\delta}_{i \cdot l}\right)=\frac{2(u-1)}{u r} \sigma_{b}^{2}=\frac{2}{r} \frac{u-1}{u} \sigma_{b}^{2}
$$

- qual \& un resultado idêntico aquele encontrado por CHAKRABARTI (1962), para essa varianicia, para os experimentos em parcelas subdivididas instalados em blocos casualizados, e por IEMMA (1981), para os experimentos em blocos incompletos bailanceados. Conforme relatado por IEMMA (1981), este resultado e contrastante com aquele de PIMENTEL GOMES (1985), que apresentou como variancia para esse contraste uma combinaçăo linear entre os residuos (a) e (b), quando o experimento está delineado en blocos casualizados. Mas, esses resultados são distintos devido ao fato que PIMENTEL GDMES (1985) usou contrastes entre médias e não entre efeitos estimados. 
90.

\section{EXEMPLOS ILUSTRATIVOS}

Para ilustrar a metodologia aplicada, utilizou-se dos dados provenientes dos seguintes exemplos ilustrativos.

1을 Exemplo - Experimento em PBIB(2)

Os dados apresentados na Tabela 4, referem-se a produção de plántulas de milho, em gramas/parcela, obtidos de um experimento instalado em parcelas subdivididas em PBIB(2), com seis linhagens de milho, a nivel de parcelas, submetidas à três níveis de saturaça de aluminio, a nivel de subparcelas [Dados adaptados de MAGNAVACA et alii (1986)].

- Os números referentes às linhagens representam os tratamentos principais, e os valores relativos aos niveis de saturação de aluminio os tratamentos secundários. Este delineamento é um PBIB(2), cujos tipos de associados são:

Tratamento Principal $1^{08}$ Associados $2^{\circ \mathrm{O}}$ Associados

1
2
3
4
5
6

2

1

4

3

6

5
$3,4,5,6$

$3,4,5,6$

$1,2,5,6$

$1,2,5,6$

$1,2,3,4$

$1,2,3,4$ 
Tabela 3 - Produção de plântulas de milho, em gramas/parce1a, do primeiro exemplo.

\begin{tabular}{|c|c|c|c|c|}
\hline \multirow{2}{*}{ Blocos } & \multirow{2}{*}{ Linhagens } & \multicolumn{3}{|c|}{ Niveis de saturação de alumínio } \\
\hline & & 1 & 2 & 3 \\
\hline \multirow[t]{3}{*}{1} & 1 & 127,9 & 102,4 & 86,4 \\
\hline & 2 & 156,9 & 113,8 & 101,0 \\
\hline & 3 & 115,9 & 102,5 & 108,9 \\
\hline \multirow[t]{3}{*}{2} & 3 & 131,0 & 121,1 & 99,6 \\
\hline & 4 & 162,1 & 121,2 & 87,3 \\
\hline & 5 & 156,5 & 90,7 & 78,0 \\
\hline \multirow[t]{3}{*}{3} & 2 & 154,2 & 135,9 & 115,0 \\
\hline & 5 & 133,8 & 101,1 & 91,7 \\
\hline & 6 & 181,9 & 70,7 & 78,4 \\
\hline \multirow[t]{3}{*}{4} & 1 & 138,7 & 118,7 & 99,1 \\
\hline & 2 & 138,7 & 125,3 & 97,6 \\
\hline & 4 & 166,9 & 105,3 & 86,9 \\
\hline \multirow[t]{3}{*}{5} & 3 & 105,8 & 116,9 & 85,4 \\
\hline & 4 & 144,2 & 93,2 & 65,8 \\
\hline & 6 & 154,8 & 70,3 & 59,5 \\
\hline \multirow[t]{3}{*}{6} & 1 & 114,8 & 121,6 & 103,4 \\
\hline & 5 & 154,1 & 104,3 & 94,2 \\
\hline & 6 & 185,5 & 90,2 & 70,4 \\
\hline
\end{tabular}

Este delineamento, de acordo com BOSE \& NAIR (1939), um PBIB(2) que possui os seguintes parametros do primeiro tipo:

$$
v=6, b=6, k=3, r=3, \lambda_{1}=2, \lambda_{2}=1, n_{1}=1, n_{2}=4
$$

e os parametros do segundo tipo são:

$$
\mathrm{p}_{11}^{1}=0, \mathrm{p}_{12}^{1}=\mathrm{p}_{21}^{1}=0, \mathrm{p}_{22}^{1}=4, \mathrm{p}_{11}^{2}=0, \mathrm{p}_{12}^{2}=\mathrm{p}_{21}^{2}=1, \mathrm{p}_{22}^{2}=2
$$

Inicialmente é conveniente obter informaçres sobre o cumprimento das exigências do modelo, usando os testes de homogeneidade e uniformidade das matrizes de variancias e covariancias entre os tratamentos principais. 
92.

Para testar a hipótese de homogeneidade $H_{0}: \Sigma_{i}=\Sigma$ versus $H_{a}: \Sigma_{i} \neq \Sigma$ para algum $i=1,2, \ldots, 6$ obtiveram-se as estimativas das matrizes de variancias e covariancias seguintes:

\begin{tabular}{|c|c|c|c|c|}
\hline \multirow{3}{*}{$S_{1}=$} & 143,2402 & $-24,1348$ & $-31,3847$ & \multirow{3}{*}{$, \ln \left|\mathrm{S}_{1}\right|=3,4825$} \\
\hline & $-24,1348$ & 107,1211 & 90,9785 & \\
\hline & $-31,3847$ & 90,9785 & 78,1309 & \\
\hline \multirow{3}{*}{$S_{2}=$} & 96,4648 & $-17,4453$ & 48,9609 & \multirow{3}{*}{$, \ln \left|S_{2}\right|=2,6983$} \\
\hline & $-17,4453$ & 122,1699 & 75,7910 & \\
\hline & 48,9609 & 75,7910 & 85,0527 & \\
\hline \multirow{3}{*}{$S_{3}=$} & 160,8438 & 40,2109 & 75,7930 & \multirow{3}{*}{, $\ln \left|s_{3}\right|=3,8946$} \\
\hline & 40,2109 & 95,1602 & $-75,2891$ & \\
\hline & 75,7930 & $-75,2891$ & 140,0654 & \\
\hline \multirow{3}{*}{$s_{4}=$} & 143,1211 & 116,5918 & 143,6484 & \multirow{3}{*}{$, \ln \left|S_{4}\right|=2,6752$} \\
\hline & 116,5918 & 197,2031 & 143,9443 & \\
\hline & 143,6484 & 143,9443 & 151,2695 & \\
\hline \multirow{3}{*}{$S_{5}=$} & 155,5235 & $-33,9590$ & $-49,8535$ & \multirow{3}{*}{, $\ln \left|S_{5}\right|=3,4906$} \\
\hline & $-33,9590$ & 50,5625 & 61,7998 & \\
\hline & $-49,8535$ & 61,7998 & 76,0635 & \\
\hline \multirow{3}{*}{$s_{0}=$} & 281,6406 & 115,3262 & 136,3359 & \multirow{3}{*}{$, \ln \left|S_{\sigma}\right|=4,4979$} \\
\hline & 115,3262 & 129,4033 & 11,4126 & \\
\hline & 136,3359 & 11,4126 & 90,0049 & \\
\hline
\end{tabular}

A estimativa para a matriz comum de variancias e covariâncias é:

$$
S=\left[\begin{array}{rrr}
163,4724 & 32,7650 & 53,9167 \\
32,7650 & 116,9387 & 51,4395 \\
53,9167 & 51,4395 & 66,6644
\end{array}\right], \ln (S)=14,0588
$$

Aplicando o teste de $(3.5 .1)$, ten-se

$M=v(r-1) \ln |S|-(r-1) \sum_{i=1}^{6} \ln \left|s_{i}\right|=127,2271$

$A_{1}=49 / 72=0,6906$ e $\left(1-A_{1}\right) M=x_{\text {obs }}^{2}=40,64$

$f_{1}=(v-1) u(u+1) / 2=60 / 2=30 \mathrm{~g} \cdot \mathrm{I}$.

Entao, como $x_{o b s}^{2}=40,64<x_{\text {lab }}^{2}=43,8, n$ a o se rejeita $H_{0}: \Sigma_{l}=\Sigma$, portanto, nåo existen evidências, que induzam que a matriz de covariância cumüü rão possa 
93.

representar as covariancias entre tratamentos secundários dentro de cada tratamento principal.

Para a verificasão da hipótese de uniformidade $H_{0}: \Sigma=\Sigma^{*}$ versus $H_{\alpha}: \Sigma \neq \Sigma^{*}$

a estimativa da matriz uniforme 6 :

$$
\begin{aligned}
& \mathrm{U}=\left[\begin{array}{rrr}
127,9468 & 46,0404 & 46,0404 \\
46,0404 & 127,8468 & 46,0404 \\
46,0404 & 46,0404 & 127,9468
\end{array}\right], \ln |\mathrm{U}|=14,2049 \\
& \text { Aplicando o teste de }(3.5 .2) \text {, tem-se } \\
& N=-(v r-v) \ln (\operatorname{det}(S) / \operatorname{det}(U))=-12(14,0588-14,2049)=1,7537 \\
& A_{2}=1 / 8=0,125,\left(1-A_{2}\right) N=x_{\text {oba }}^{2}=1,53 \\
& f_{2}=\left(u^{2}+u-4\right) / 2=8 / 2=48.1 \text {. }
\end{aligned}
$$
rejeita $H_{0}: \Sigma=\Sigma$, portanto, não existen evidências que induzan que a matriz de covariancia nã seja do tipo uniforme.

Os efeitos de tratamentos principais $\left(\hat{t}_{i}\right)$ ajustados foram obtidos, considerando-se a expressao:

$$
\begin{aligned}
& \hat{t}_{i}=\frac{1}{u r(k-1) \Delta}\left[\left(k \Delta-u A_{2}\right) Q_{i}+u\left(A_{1}-A_{2}\right) S_{1}\left(Q_{i}\right)\right]= \\
& \hat{t}_{i}=\frac{1}{48}\left[7 Q_{i}-S_{1}\left(Q_{i}\right)\right]
\end{aligned}
$$

onde: $a_{11}=8, a_{12}=1, a_{21}=0, a_{22}=6, \Delta=48, A_{1}=12, A_{2}=6$

Sendo $\hat{\mu}=\mathrm{G} / \mathrm{vru}=6067,5 / 54=112,36$, tem-se que as medias ajustadas de tratamentos principais poden ser obtidas por $\hat{\mu}_{i}=\hat{\mu}+\hat{t}_{i}$. A analise de variancia intrablocos pode ser obtida com o auxilio da seguinte tabela auxiliar (Tabela 4). 
94.

Tabela 4 - Tabela auxiliar para a análise intrablocos do experimento em $\operatorname{PBIB}(2)$.

\begin{tabular}{rrrrrrr}
\hline $\mathbf{i}$ & $\mathrm{T}_{i}$ & \multicolumn{1}{c}{$\mathrm{A}_{i}$} & \multicolumn{1}{c}{$\mathrm{Q}_{\mathrm{i}}$} & \multicolumn{1}{c}{$\mathrm{S}_{1}\left(Q_{i}\right)$} & \multicolumn{1}{c}{$\hat{\mathrm{t}}_{i}$} & $\hat{\mathrm{m}}_{i}$ \\
\hline 1 & 1013,0 & 3131,4 & $-92,4 / 3$ & $259,6 / 3$ & $-906,2 / 144$ & 107,36 \\
2 & 1138,4 & 3155,6 & $259,6 / 3$ & $-92,4 / 3$ & $1909,6 / 144$ & 126,92 \\
3 & 987,1 & 2959,1 & $2,2 / 3$ & $78,1 / 3$ & $-62,7 / 144$ & 113,22 \\
4 & 1032,9 & 3020,6 & $78,1 / 3$ & $2,2 / 3$ & $544,5 / 144$ & 117,44 \\
5 & 1004,4 & 3148,7 & $-135,5 / 3$ & $-112,0 / 3$ & $-836,5 / 144$ & 107,85 \\
6 & 961,7 & 2997,1 & $-112,0 / 3$ & $-135,5 / 3$ & $-648,5 / 144$ & 109,15 \\
\hline Total & 6137,5 & 18412,5 & 0,0 & 0,0 & 0,0 &
\end{tabular}

$\operatorname{SQT}\left(a_{j}.\right)=\sum_{i=1}^{\infty} \hat{t}_{i} Q_{i}=\frac{807848,78}{432}=1870,020$

o quadro da añalise de variância intrablocos é:

\begin{tabular}{lcccc}
\hline V.C.M. & G.L. & S.Q. & Q.M. & $F$ \\
\hline Blocos & 5 & 2395,765 & 479,153 & \\
Trat. Prin.(T)(aj.) & 5 & 1870,020 & 374,004 & $5,51^{+}$ \\
Residuo(a) & 7 & 475,408 & 67,915 & \\
\hline Parcelas & 17 & 4741,193 & & \\
\hline Tra. Sec.(T $\left.{ }^{*}\right)$ & 2 & 30271,145 & 15135,572 & $184,79^{+}$ \\
Tx T* & 10 & 10871,837 & 1087,184 & $13,27^{++}$ \\
Residuo(b) & 24 & 1965,744 & 81,906 & \\
\hline Total & 53 & 47849,911 & & \\
\hline
\end{tabular}

,+++ Significativo ao nivel de $5 \%$ e $1 \%$ de probabilidade respectivamente.

As estimativas das variâncias das estimativas dos contrastes elementares entre médias de tratamentos, obtidas de acordo com (3.12), são:

i) Para contrastes entre duas médias de tratamentos principais tidos como primeiros associados:

$\hat{\operatorname{Var}}\left(\hat{\mathfrak{m}}_{i}-\hat{\mathfrak{m}}_{i}\right)=2 \frac{\mathrm{k} \Delta-\mathrm{uA}}{\operatorname{ur}(\mathrm{k}-1) \Delta} \operatorname{QMR}(\mathrm{a})=\frac{1}{4} \operatorname{QMR}(\mathrm{a})=16,979$ 
ii) Para contrastes entre duas medias de tratamentos principais tidos como segundos associados:

$\operatorname{Var}\left(\hat{m}_{i}-\hat{m}_{i}\right)=2 \frac{k \Delta-\mathrm{uA}_{2}}{\operatorname{ur}(k-1) \Delta} \operatorname{QMR}(\mathrm{a})=\frac{7}{24} \operatorname{QMR}(\mathrm{a})=19,809$

iii) Para contrastes entre duas médias de tratamentos secundários:

$\operatorname{Var}\left(\bar{y} \ldots l^{-\bar{y}} \ldots l\right)=\frac{2}{\operatorname{Vr}} \operatorname{QMR}(b)=\frac{1}{9} \operatorname{QMR}(b)=9,101$

iv) Para contrastes entre duas medias de tratamentos secundários, em um dado tratamento principal

$\operatorname{Var}\left(\bar{y}_{i L}-\bar{y}_{i l}\right)=\frac{2}{r} \operatorname{QMR}(b)=\frac{2}{2} Q M R(b)=81,906$

v) Para contrastes entre duas medias de tratamentos principais, em un dado tratamento secundário

$\operatorname{Var}\left(\bar{y}_{i l}-\bar{y}_{i l}\right)=\frac{2}{r} \frac{\operatorname{QMR}(a)+(u-1) Q M R(b)}{u}=77,242$

vi) Para efeitos estimados de dois tratamentos secundários, em um dado tratamento principal:

$\operatorname{Var}\left(\hat{\delta}_{i l}-\hat{\delta}_{i l}\right)=\frac{2(V-1)}{V r} \operatorname{QMR}(b)=\frac{5}{9} \operatorname{QMR}(b)=44,503$

vii) Para efeitos estimados entre dois tratamentos principais, em um dado tratamento secundário:

$\hat{\operatorname{Var}}\left(\hat{\delta}_{i L}-\hat{\delta}_{i, l}\right)=\frac{2(u-1)}{\mathrm{Ur}} \mathrm{QMR}(b)=\frac{4}{9} \mathrm{QMR}(b)=36,403$

2- Exemplo - Experimento em reticulados quadrados

Os dados apresentados na Tabela 5 , referem-se

a produção de grãos de cultivares de milho, em $\mathrm{kg} / \mathrm{ha}$, obtidos de um experimento instalado em parcelas subdivididas, com as cultivares, a nivel de parcelas, dispostas em PBIB(2) do tipo reticulado. guadrado, onde em cada cultivar foram testados três niveis de endogamia [Dados adaptados de GAMA et alii, 1985]. 
96.

Tabela 5 - Produção de grãos de algumas cultivares de milho, em $\mathrm{kg} / \mathrm{ha}$, do segundo exemplo.

\begin{tabular}{|c|c|c|c|c|c|}
\hline Repetiçăo & Bloco & Cultivar & \multicolumn{3}{|c|}{ Nivel de endogamia } \\
\hline \multirow[t]{9}{*}{1} & 1 & 1 & 6461 & 5271 & 5995 \\
\hline & & 2 & 7128 & 4522 & 5131 \\
\hline & & 3 & 5490 & 3575 & 4174 \\
\hline & 2 & 4 & 5697 & 5879 & 4572 \\
\hline & & 5 & 5262 & 4275 & 5477 \\
\hline & & 6 & 5262 & 4255 & 5477 \\
\hline & 3 & 7 & 5404 & 3838 & 3420 \\
\hline & & 8 & 5386 & 5608 & 3944 \\
\hline & & 9 & 4872 & 3075 & 4064 \\
\hline \multirow[t]{9}{*}{2} & 1 & 1 & 5628 & 6434 & 4613 \\
\hline & & 4 & 4517 & 5255 & 5336 \\
\hline & & 7 & 5804 & 5122 & 2925 \\
\hline & 2 & 2 & 7322 & 5046 & 4471 \\
\hline & & 5 & 4420 & 5475 & 4693 \\
\hline & & 8 & 5981 & 4484 & 3348 \\
\hline & 3 & 3 & 4720 & 4805 & 3649 \\
\hline & & 6 & 4420 & 5475 & 4267 \\
\hline & & 9 & 4023 & 2847 & 2782 \\
\hline \multirow[t]{9}{*}{3} & 1 & 1 & 5226 & 5181 & 5116 \\
\hline & & 5 & 4088 & 4185 & 4167 \\
\hline & & 9 & 3582 & 4150 & 3291 \\
\hline & 2 & 2 & 6295 & 5754 & 5694 \\
\hline & & 6 & 4088 & 3985 & 4593 \\
\hline & & 7 & 6604 & 3951. & 4228 \\
\hline & 3 & 3 & 4278 & 3736 & 4931 \\
\hline & & 4 & 5942 & 4551 & 4098 \\
\hline & & 8 & 6799 & 4345 & 4776 \\
\hline
\end{tabular}

Esse delineamento experimental corresponde a um PBIB(2) do tipo quadrado latino, conhecido como reticulado quadrado triplo, cujos tipos de associados são: 
Tratamento Principal $1^{08}$ Associados $2^{08}$ Associados

\begin{tabular}{|c|c|c|c|c|c|c|}
\hline 1 & 2 & 3 & 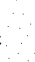 & & & \\
\hline 2 & 1 & 3 & & & & 4 \\
\hline 3 & 1 & 2 & & & & 5 \\
\hline 4 & 1 & 3 & & 3 & 7 & 2 \\
\hline 3 & 1 & 2 & & D & & 3 \\
\hline 6 & 2 & 3 & & & 7 & 1 \\
\hline 7 & 1 & 2 & & 3 & 3 & 3 \\
\hline 8 & 2 & 3 & & & & 1 \\
\hline 9 & 1 & 3 & & 6 & 7 & 2 \\
\hline
\end{tabular}

De acordo com BOSE \& NAIR (1939), os parametros do primeiro tipo são:

$$
v=9, b=9, r=3, k=3, \lambda_{1}=1, \lambda_{2}=0, n_{1}=6, n_{2}=2
$$

e os parámetros do segundo tipo são:

$$
\begin{aligned}
p_{11}^{1}=3, & p_{12}^{1}=p_{21}^{1}=2, p_{22}^{1}=0, p_{11}^{2}=6, p_{12}^{2}=p_{21}^{2}=0, p_{22}^{2}=1 \\
& \text { Os efeitos de tratamentos principais }\left(\hat{t}_{i}\right)
\end{aligned}
$$

ajustados foram obtidos considerando-se a expressão:

$$
\begin{aligned}
& \hat{t}_{i}=\frac{1}{u r(k-1) \Delta}\left[\left(k \Delta-u A_{1}\right) Q_{i}+u\left(A_{2}-A_{1}\right) S_{2}\left(Q_{i}\right)\right]= \\
& \hat{t}_{i}=\frac{1}{54}\left[8 Q_{i}-S_{2}\left(Q_{i}\right)\right]
\end{aligned}
$$

onde: $a_{11}=9, a_{12}=0, a_{21}=-2, a_{22}=6, \Delta=54, A_{1}=6, a_{2}=0$

A análise de variancia intrablocos pode ser desenvolvida com o auxilio da Tabela 6.

Sendo $\hat{\mu}=G / v r u=389010 / 81=4802,59$, tem-se que as medias ajustadas de tratamentos principais podem ser obtidas por $\hat{\mu}_{i}=\hat{\mu}_{t} \hat{t}_{i}$. 
98.

Tabela 6 - Tabela auxiliar para a analise de variancia intrablocos do segundo exemplo considerado.

\begin{tabular}{lrrrrrr}
\hline$i$ & $T_{i}$ & $A_{i}$ & $Q_{i}$ & $S_{1}\left(Q_{i}\right)$ & $S_{2}\left(Q_{i}\right)$ & $t_{i}$ \\
\hline 1 & 49925 & 132367 & $17408 / 3$ & -6748 & $2836 / 3$ & $45476 / 54$ \\
2 & 51363 & 138179 & $15910 / 3$ & -226 & $-15232 / 3$ & $47504 / 54$ \\
3 & 39358 & 128191 & $-10117 / 3$ & 6974 & $-10805 / 3$ & $-23377 / 54$ \\
4 & 45847 & 135246 & $2295 / 3$ & -226 & $-1617 / 3$ & $6659 / 54$ \\
5 & 42042 & 130382 & $-4256 / 3$ & 6974 & $-16666 / 3$ & $-5794 / 54$ \\
6 & 41822 & 128336 & $-2870 / 3$ & -6748 & $23114 / 3$ & $-15358 / 54$ \\
7 & 41296 & 130437 & $-6549 / 3$ & 6974 & $-14373 / 3$ & $-12673 / 54$ \\
8 & 44671 & 128307 & $5706 / 3$ & -6748 & $14538 / 3$ & $10370 / 54$ \\
9 & 32686 & 115585 & $-17527 / 3$ & -226 & $18205 / 3$ & $-52807 / 54$ \\
\hline
\end{tabular}

3890101167030

0

0

0

0

As somas de quadrados são:

$\operatorname{SQT}(a j)=.\sum_{i=1}^{o} \hat{t}_{i} Q_{i}=\frac{2935674072}{162}=18121445$

$\mathrm{SQB}=\frac{1}{\mathrm{ku}} \sum_{j=1}^{b} B_{j}^{2}-\operatorname{Cor}=12528385$

SQT ot $=\sum_{i j i} y_{i j l}^{2}-\operatorname{Cor}=77648510$

SQPar $=\frac{1}{u} \sum_{i j} y_{i j}^{2} .-\operatorname{Cor}=34199043$

$\operatorname{SQR}(a)=\operatorname{SQPar}-\operatorname{SQB}-\operatorname{SQT}(\mathrm{aj})=$.

$\operatorname{SQT}^{*}=\frac{1}{\operatorname{vr}} \sum_{l=1}^{u} \mathrm{y}^{2} \ldots l-\operatorname{Cor}=13181480$

SQ $\mathrm{TXT}^{*}=12479439$

$\mathrm{SQR}(\mathrm{b})=\mathrm{SQTotal}-\mathrm{SQPar}-\mathrm{SQT}^{*}-\mathrm{SQT} \mathrm{TXT}^{*}=17788547$

A soma de quadrados de blocos pode ser decomposta em duas partes: soma de quadrados de repetiça (SQR) e soma de quadrados de blocos dentro de repetiça (SQB/R), as quais são:

$$
\begin{aligned}
& S Q R=\frac{1}{v U} \sum_{j=1}^{r} R_{j}^{2}-\operatorname{Cor}=821856 \\
& S Q B / R=S Q B-S Q R=11706529
\end{aligned}
$$


Desse modo, o quadro de análise de varância é:

\begin{tabular}{lrrrr}
\hline V.C.M. & G.L. & S.Q. & Q.M. & F \\
\hline Blocos & 8 & 12528385 & 1566048 & \\
Repet. & 2 & 821856 & 410928 & \\
$\quad$ Blocos/Rep. & 6 & 11706529 & 1951088 & \\
Trat. Prin.(T)(aj.) & 8 & 18121445 & 2265181 & $6,38^{++}$ \\
Residuo(a) & 10 & 3549213 & 354921 & \\
Trat. Sec.(T $\left.{ }^{*}\right)$ & 2 & 13181480 & 6590740 & $13,34^{++}$ \\
T x T & 16 & 12479439 & 779965 & 1,58 \\
Residuo(b) & 36 & 17788547 & 484126 & \\
\hline Total & 80 & 77648510 & & \\
\hline
\end{tabular}

++ Significativo ao nivel de $1 \%$ de probabilidade.

As estimativas das variancias das estimativas dos contrastes elementares entre médias de tratamentos, obtidas de acordo com ( 3.12 ), são:

i) Um contraste entre duas medias de tratamentos principais, tidos como primeiros associados:

$\operatorname{Var}\left(\hat{m}_{i}-\hat{m}_{i}\right)=2 \frac{k \Delta-u A_{1}}{\operatorname{ur}(k-1) \Delta} \operatorname{QM}(a)=\frac{8}{27} \operatorname{QM}(a)=105161,78$

ii) Un contraste entre duas médias de tratamentos principais tidos como segundos associados:

$\operatorname{Var}\left(\hat{m}_{i}-\hat{m}_{i}\right)=2 \frac{k \Delta-u A_{2}}{\operatorname{ur}(k-1) \Delta} \operatorname{QM}(a)=\frac{1}{3} \operatorname{QM}(a)=118307,00$

iii) Um contraste entre duas médias de tratamentos secundarios:

$\operatorname{Var}\left(\hat{m}_{l}-\hat{m}_{l}\right)=\frac{2}{\operatorname{Vr}} \operatorname{QM}(b)=\frac{2}{27} Q M(b)=36601,93$

iv) Um contrastes entre duas medias de tratamentos secundários, em um dado tratamento principal

$\hat{\operatorname{Var}}\left(\bar{y}_{i l}-\bar{y}_{i l} \cdot\right)=\frac{2}{r} \operatorname{QMR}(b)=\frac{2}{3} \operatorname{QMR}(b)=329417,33$ 
100.

v) Um contrastes entre duas mødias de tratamentos principais em um dado tratamento secundario

$\hat{\operatorname{Var}}\left(\bar{y}_{i L}-\bar{y}_{i}{ }_{i}\right)=\frac{2}{r} \frac{\operatorname{QMR}(\mathrm{a})+(\mathrm{u}-1) Q M R(b)}{u}=298482,89$

vi) Um contraste entre efeitos estimados de dois tratamentos secundários, em um dado tratamento principal:

$\operatorname{Var}\left(\hat{\delta}_{i l}-\hat{\delta}_{i l}\right)=\frac{2(v-1)}{V r} \operatorname{QM}(b)=\frac{16}{27} Q M(b)=282815,41$

vii) Um contraste entre efeitos estimados de dois tratamentos principais, em um dado tratamento secundário:

$\operatorname{Var}\left(\hat{\delta}_{i l}-\hat{\delta}_{i \cdot l}\right)=\frac{2(\mathrm{u}-1)}{u r} Q M(b)=\frac{4}{3} Q M(b)=218611,56$ 


\section{CONCLUSŌES}

0 modelo matemático apresentou-se adequado com relaçăo aos objetivos propostos, permitindo testar as hipóteses básicas de nulidade sobre os efeitos de tratamentos principais ou primários, tratamentos secundários e interaçăo.

Os efeitos de tratamentos principais ajustados, em delineamento em blocos incompletos parcialmente balanceado (PBIB), foram estimados de modo análogo àqueles em blocos incompletos balanceados.

Os efeitos estimados de tratamentos secundários e interação foram obtidos como nos ensaios com parcelas subdivididas em blocos casualizados.

A primeira parte da análise de variancia envolvendo blocos, tratamentos principais e residuo(a), é semelhante às análises em blocos incompletos balanceados. A segunda parte, envolvendo tratamentos secundários, interação e residuo(b) é semelhante às análises com parcela.s subdivididas em blocos casualizados.

Se $\lambda_{1}=\lambda_{2}=\ldots=\lambda_{m}=\lambda$ recai-se em uma análise em blocos incompletos balancedos e, se $b=r, a$ análise será de acordo com parcelas subdivididas em blocos casualizados, ficando, assim, caracterizados como casos particulares do modelo prosposto.

Os testes $\mathrm{F}$ para tratamentos principais, tratamentos secundários e interaçăo são exatos, enquanto, para tratamentos principais dentro de um dado tratamento secundário não existe um teste exato.

A precisăo da estimaitiva lit üin contraste entre 
102 .

duas médias de tratamentos principais, depende da classe de associados que esses tratamentos pertencem.

As funçós lineares entre efeitos de tratamentos principais, dado un mesmo tratamento secundário, nem sempre são estimáveis. Serão estimáveis aquelas funçđes provenientes de combinaçes lineares das observaçชes presentes no mesmo bloco. 
103.

\section{REFERENCIAS BIBLIOGRAFICAS}

ANDERSON, R.L. \& BANCROFT, T.A. Statistical theory in research. New York, MacGraw-Hill, 1952. 399p.

BARBOSA, G.V.S. Planejanento e análise de ensaios em blocos incompletos parcialmente balanceados com duas classes de associados - PBIB (2). Piracicaba, 1986. 118p. (Mestrado - Escola Superior de Agricultura "Luiz de Queiroz"/ USP).

BOSE, R.C.; CLATHORTHY, W.H.; SHRIKHANDE, S.S. Tables of partially balanced designs with two associate classes. North Carolina Agricultural Experimental Bulletin, no. 107, Ralejgh, 1954. 255p.

BOSE, R.C. \& CONNOR, W.S. Combinatorial properties of group divisible incomplete block designs. Annals of Mathematacal Statistics, Baltimore, 23: 367-83, 1952.

BOSE, R.C. \& NAIR, K.R. Partially balanced incomplete block design. Sankhya, Calcutta, 4: 337-72, 1939.

BOSE, R.C. \& SHIMAMOTO, T. Classification and analysis of partially incomplete block designs with two associate. Journal of the American Statistical Association, Washington, 47: 151-84, 1952.

CAMPOS, H. Estatistica aplicada a experimentação com cana de açucar. São Paulo, Fundação de Estudos Agrários Luiz de Queiroz, 1984. 292p.

CHAKRABARTI, M.C. Mathematics of design and analysis of experiments. Bombay, Asia Publishing House, 1962. 120p. 
104.

CLATWORTHY, W.H. Some new families of partially balanced designs of the latin square type and related designs. Technometrics, Hashington, 9(2): 229-44, 1967.

COCHRAN, W.G. \& COX, G.M. Experimental designs. 2.ed. New York, John Wiley \& Sons, 1957. 611p.

CONDE, A.R. Estudo dos componentes de variancia nos experimentos em parcelas subdivididas. Piracicaba, 1974. 57p. (Mestrado - Escola Superior de Agricultura "Luiz de Queiroz"/USP).

DANFORD, M.B.; HUGHES, H.M. \& McNEE, R.C. On the analysis of repeated measurements experiments. Biometrics, Raleigh, 16: 547-65, 1960 .

DAS, M.N. \& GIRI, N.C. Design and analysis of experiments. 2.ed. New York, John Wiley, 1986. 488p.

DEY, A. Some new partially balanced designs with two associate classes. Sankhyrã, Calcutta, 50: 90-94, 1988.

FEDERER, W.T. Experimental design; theory and application. New York, The MacMillan, 1955. 544p.

GAMA, E.E.G.; VIANNA, R.T.; NASPOLINI EILHO, V. \& MAGNAVACA, R. Efeito depressivo da endogamia em geraçós avançadas de quatro tipos geneticos de híbridos de milho. Pesquisa agropecuăria brasileira, Brasilia, 20(11): 1293-300, 1985.

GILL, L.G. Design and analysis of experiments in the animal and medical sciences. Anes, The Iowa State University Press, 1978. 3v.

GRAYBIL, F.A. An introduction to linear statistical models. New York, McGraw-Hill, 1961. 463p.

GRAYBILL, F.A. Theory and application of the Iinear models. 2.ed. Massachusetts, Duxbury Press, 1976. 703p.

HALPERIN, M. Normal regression theory in the presence of intra-class correlation. Annals of Mathematical Statistire, Baltimnre: 22: 573-80, 1951. 
HARTER, H.L. On the analysis of split-plot experiments. Biometrics, Raleigh, 17(1): 144-9, 1961.

HICKS, C.R. Fundamental concepts in the design of experiments. 2.ed. New York, Holt, Rinehart an Winston, 1973. $349 p$.

IEMMA, A.F. Análise de experimentos em parcelas subdivididas com tratamentos principais dispostos em blocos incompletos balanceados. Piracicaba, 1981. 145p. (Doutorado - Escola Superior de Agricultura "Luiz de Queiroz"/USP).

IEMMA, A.F. Testes de hipóteses nos experimentos com parcelas subdivididas em blocos incompletos balanceados. Desarrollo Rural en las Americas, San Jose, 15(2): 143$151,1983$.

IEMMA, A.F. Modelos lineares; uma introducão para profissionais da pesquisa agropecuária. Londrina, Departamento de Matemática Aplicada/UEL, 1987. 263p.

IEMMA, A.F. Matrizes para estatistica; um texto para profissionais de ciencias apiicadas: Piracicaba, ESALQ, Departamento de Matemática e Estatı́stica, 1988. 339p.

JOHN, P.W.K. Incomplete block designs. New York, Marcel Dekker, 1980. 101,

KAGEYAMA, S. Connectedness of two-associates PBIB designs. Journal of Statistical Planning and Inference, Amsterdan, 7: 77-82, 1982 .

KEMPTHORNE, O. Recent developments in the design of field experiments. IV. Lattice squares with split plots. Journal of Agriculture Science, Cambridge; 37: 156-62, 1947.

KEMPTHORNE, 0 . The design and analysis of experiments. New York, John Wiley, 1952. 631p.

LEAL, M.L.S. Análise de dados experimentais com medidas repetidas. Brasilia, 1979. 99p. (Mestrado - Universidade Federal de Brasilia). 
LEONARD, W.H. \& CLARK, A.G. Field plot technique. Minneapolis, Burgess, 1939. 288p.

LITTLE, T.M. \& HILLS, J.F. Agricultural experimentation; design and analysis. New York, John Wiley, 1978. 349p.

MAGNAVACA, R.; BAHIA FILHO, A.F.C.; LOPES, M.A.; GAMA, E.E. G. Avaliaça de híbridos triplos de milho para solos de cerrado. Rel. tec. anu. CNPMS, Sete Lagoas, 3: 40-1, 1986.

McELROY, F.H. A necessary an sufficient condition that ordinary least-squares estimators be best linear unbiased. Journal of the American Statistician Association, Washington, 62(320): 1302-04, 1967.

MILLIKEN, G.A. \& JOHNSON, D.E. Analysis of messy data; designed experiments. New York, Van Nostrand Reinhold. 1984. 485p.

MIRANDA FILHO, J.B.; SOUZA JR., C.L. \& GERALDI, I.O. Avaliação de híbridos so $x$ So entre duas variedades de milho. In: CONGRESSO NACIONAL DE MILHO E SORGO, 15., Maceio, 1984. Anais. Brasilia, EMBRAPA-DDT, 1986. p. 177-81.

MOORE, W. \& BLISS, C.I. A method for determining insecticidal effectiveness using Aphis rumicis and certain organic compounds. Journal of Economic Entomology, Menaska, 35: $544-53,1942$.

NAIR, K.R. Analysis of partially balanced incomplete block designs ilustrated on the simple square and rectangular lattices. Biometrics, Raleigh, 8: 122-55, 1952.

NIGAM, A.K.; PURI, P.D. \& GUPTA, V.K. Characterizations and analysis of block designs. New York, John Wiley, 1989. 176p.

NOGUEIRA, M.C.S. Curso de estatística experimental aplicada a experimentaçæo agronsmica. Piracicaba, ESALQ-DME, 1991. 168p. 
OGAWA, J. Statistical theory of analysis of experimental designs. New York, Marcel Dekker, 1974. 465p:

OLIVEIRA, A.C. de Análise intrablocos de experimentos em blocos incompletos parcialmente balanceados com alguns tratamentos comuns adicionados em cada bloco. Piracicaba, 1985. 153p. (Doutorado - Escola Superior de Agricultura "Luiz de Queiroz"/USP).

PEARSON, E.S. Some comments on the accuracy of Box's approximations to the distribution of $M$. Biometrika, Londres, 5e: $219-20,1969$.

PIMENTEL GOMES, F. Método geral de análise para delineamentos em quadrados reticulados. Seminarios de Estatistica, Piracicaba, $10: 33-54,1954$.

PIMENTEL GOMES, $\mathrm{F}$. The solution of normal equations of experiments in incomplete blocks. Ciencia e Cultura, sao Paulo, 20: $733-46,1968$.

PIMENTEL GOMES, F. Curso de estat1stica experimental. 11.ed. Piracicaba, Livraria Nobe1, 1985. 466p.

PINHO, S.Z. Estudo dos componentes de variancia nos experimentos em parcelas subdivididas com uma observação perdida. Piracicaba, 1975. 45p. (Mestrado - Escola Superior de Agricultura "Luiz de Queiroz"/USP).

RAGHAYARAO, D. Constructions and combinatorial problems in destgns of experiments. New York, John wiley, 1971. $386 \mathrm{p}$

RAO, C.R. On the linear combination of observations and the general theory of least squares. Sankhya, Calcutta, 7 : $237-56,1946$.

RAO, C.R. General methods of analysis for incomplete block designs. Journal of the American Statistical Association, washington, 42: 541-61, 1947. 
RAO, C.R. Linear statistical inference and its applicatlons. New York, John Wiley, 1973. 625p.

REGAZZI, A.J. Análise de experimentos em parcelas subdivididas com tratamentos secundários em apenas alguns dos tratamentos principais. Piracicaba, 1984. 105p. (Doutorado - Escola Superior de Agricultura "Luiz de Queiroz"/USP).

RIBOLDI, J. A decomposição da soma de quadrados de tratamentos nos delineamentos em blocos incompletos parcialmente balanceados. Piracicaba, 1988. 165p. (Doutorado - Escola Superior de Agricultura "Luiz de Queiroz"/USP). ROBINSON, J. Incomplete split plot designs. Biometrics, Raleigh, 23: 793-802, 1967.

SANCHES, S.F. Análise de experimentos em parcelas subdivididas, em blocos casualizados, com perda de uma ou mais subparcelas ou parcelas. Piracicaba, 1980. 119p. (Doutorado - Escola Superior de Agricultura "Luiz de Queiroz"/USP).

SCARIANO, S.M. \& DAVENPORT, J.M. The effects of violations of independence assumptions in the one-way ANOVA. The American Statisticlan, wastington, $41(2): 123-9,1987$.

SEARLE, S.R. Linear models. New York, John Wiley, 1971. $532 \mathrm{P}$.

SEARLE, S.R. Linear models for unbalanced data. New York, John Wiley, 1987 . 536p.

STEEL, R.G.D. \& TORRIE, J.H. Principles and procedures of statistics. Nova York, McGraw-Hill, 1960. 593p.

VIZUNI, E. Análise de experimentos em blocos casualizados completos aumentados (blocos de Federer), com parcelas subdivididas no tempo. Piracicaba, 1984. 125p. (Mestrado - Escola Superior de Agricultura "Luiz de Queiroz" (USP). 
109.

WILKS, S.S. Sample criteria for testing equality of means, equality of variances, and equality of covariances in a normal multivariate distribution. The Annals of Mathematics Statistics, Ann Arbor, 17: 257-81, 1946.

YATES, $F$. A new method of arranging variety involving a large number of varieties. Journal of Agricultural Science, Cambridge, 26: 424-55, 1936. 
APENDICE 1 - Núcleos das formas quadráticas

Aqui estão relacionadas as matrizes dos projetores ortogonais que forneceram as somas de quadrados para a análise da variância. Com finalidade didatica, foram considerados os projetores do exemplo ilustrativo do item (3.1).

$$
Q_{1}=\frac{1}{v r u}\left[\begin{array}{llllllll}
E & E & E & E & E & E & E & E \\
E & E & E & E & E & E & E & E \\
E & E & E & E & E & E & E & E \\
E & E & E & E & E & E & E & E \\
E & E & E & E & E & E & E & E \\
E & E & E & E & E & E & E & E \\
E & E & E & E & E & E & E & E \\
E & E & E & E & E & E & E & E
\end{array}\right]_{v r u}
$$

onde $E$ uma sub-matriz de uns, de dimensão $(u \times u)$.

$$
Q_{2}=\frac{1}{v r u}\left[\begin{array}{llllllll}
B_{1} & B_{1} & -E & -E & -E & -E & -E & -E \\
B_{1} & B_{1} & -E & -E & -E & -E & -E & -E \\
-E & -E & B_{1} & B_{1} & -E & -E & -E & -E \\
-E & -E & B_{1} & B_{1} & -E & -E & -E & -E \\
-E & -E & -E & -E & B_{1} & B_{1} & -E & -E \\
-E & -E & -E & -E & B_{1} & B_{1} & -E_{1} & -E^{\prime} \\
-E & -E & -E & -E & -E & -E_{1} & B_{1} & B_{1} \\
-E & -E & -E & -E & -E & -E & B_{1} & B_{1}
\end{array}\right]_{\text {vru }}
$$

onde: $B_{1}$ E uma sub-matriz com as elementos $(b-1)$, de dimens8es $(u \times u)$;

E ó uma sub-matriz de uns de dimensão ( $u \times u)$. 


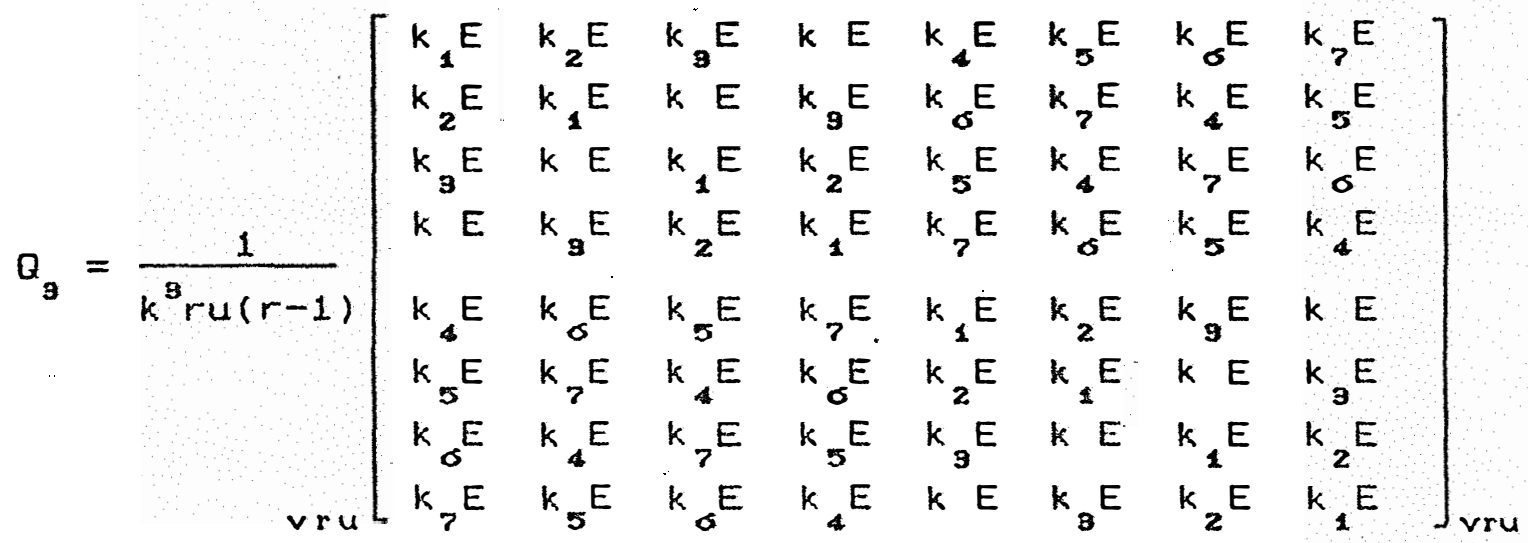

$$
\text { onde; } \begin{array}{rlrl}
k_{1} & =k(k-1)(r-1)(k+1) ; & k_{2}=-k(r-1)(k+1) ; \\
k_{9} & =k(k-1) ; & k_{4}=(k-1)^{2}[(r-1)(k+1)-1] ; \\
k_{5} & =-(k-1)[(r-1)(k+1)-1] ; & \\
k_{6} & =-(k-1)[(r-1)(k+1)-1] ; k_{7}=(r-1)(k+1)-1 ; \\
E & \text { E uma submatriz de uns, de dimensores }(u \times u) .
\end{array}
$$

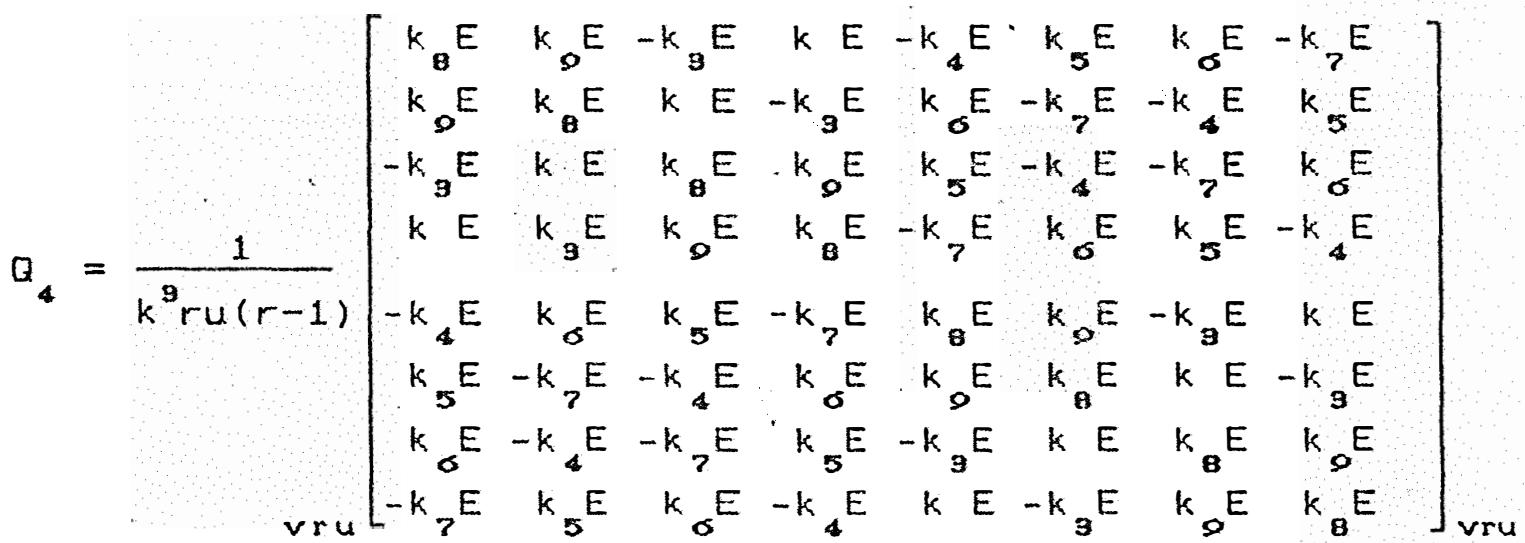

$$
\text { onde: } \begin{array}{rlrl}
k_{0} & =k(k-1)(r-1)(k r-k-1) ; & k_{0}=-k(r-1)(k r-k-1) ; \\
k_{9}=k(k-1) ; & k_{0}=(k-1)^{2}[(r-1)(k+1)-1] ; \\
k_{5}=-(k-1)[(r-1)(k+1)-1] ; & & \\
k_{0}=-(k-1)[(r-1)(k+1)-1] ; & k_{7}=(r-1)(k+1)-1 ; \\
E^{6} & \text { e uma.submatriz de uns, de dimensठ̈es }(u \times u) .
\end{array}
$$




$$
Q_{5}=\frac{1}{v r u}\left[\begin{array}{lllllllll}
u_{1} & u_{1} & u_{1} & u_{1} & u_{1} & u_{1} & u_{1} & u_{1} \\
u_{1} & u_{1} & u_{1} & u_{1} & u_{1} & u_{1} & u_{1} & u_{1} \\
u_{1} & u_{1} & u_{1} & u_{1} & u_{1} & u_{1} & u_{1} & u_{1} \\
u_{1} & u_{1} & u_{1} & u_{1} & u_{1} & u_{1} & u_{1} & u_{1} \\
u_{1} & u_{1} & u_{1} & u_{1} & u_{1} & u_{1} & u_{1} & u_{1} \\
u_{1} & u_{1} & u_{1} & u_{1} & u_{1} & u_{1} & u_{1} & u_{1} \\
u_{1} & u_{1} & u_{1} & u_{1} & u_{1} & u_{1} & u_{1} & u_{1} \\
u_{1} & u_{1} & u_{1} & u_{1} & u_{1} & u_{1} & u_{1} & u_{1}
\end{array}\right]_{v r u}
$$

onde $U_{\alpha}$ é uma submatriz uniforme, de dimensóes $(u \times u)$, com os elementos $(u-1)$ na diagonal principal e -1 fora da diagonal.

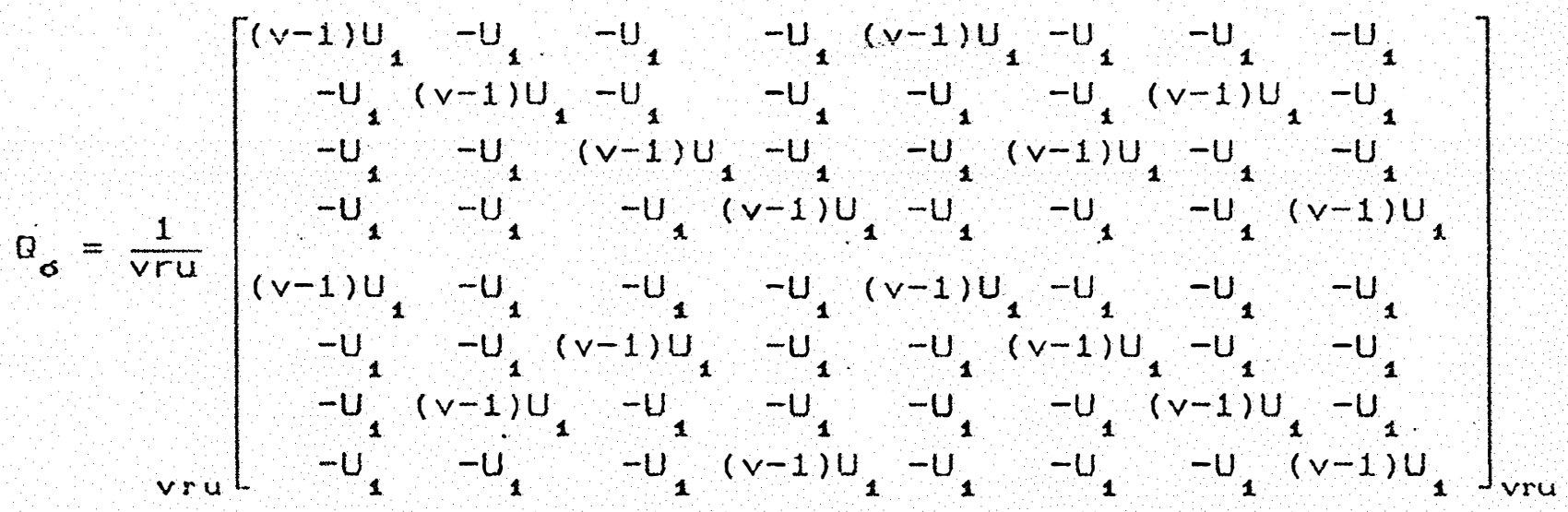

onde $U_{1}$ \& uma sub-matriz uniforme, de dimenşes $(u \times u)$, com os elementos $u-1$ na diagonal principal e -1 fora da diagonal. 
113

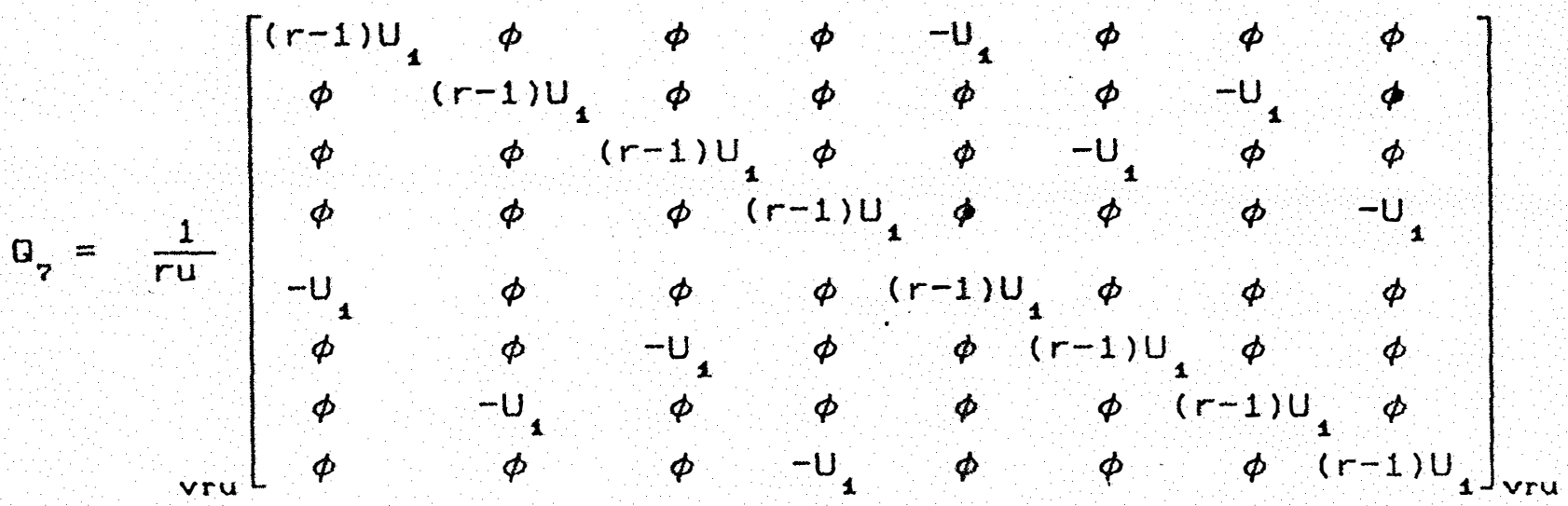

onde: $\phi$ e uma submatriz de zeros, de dimensoes $(u \times u)$;

$U_{1}$ \& uma submatriz uniforme de dimensobs $(u x u)$, com os elementos $u_{-1}$ na diagonal principal e -1 fora da diagonal.

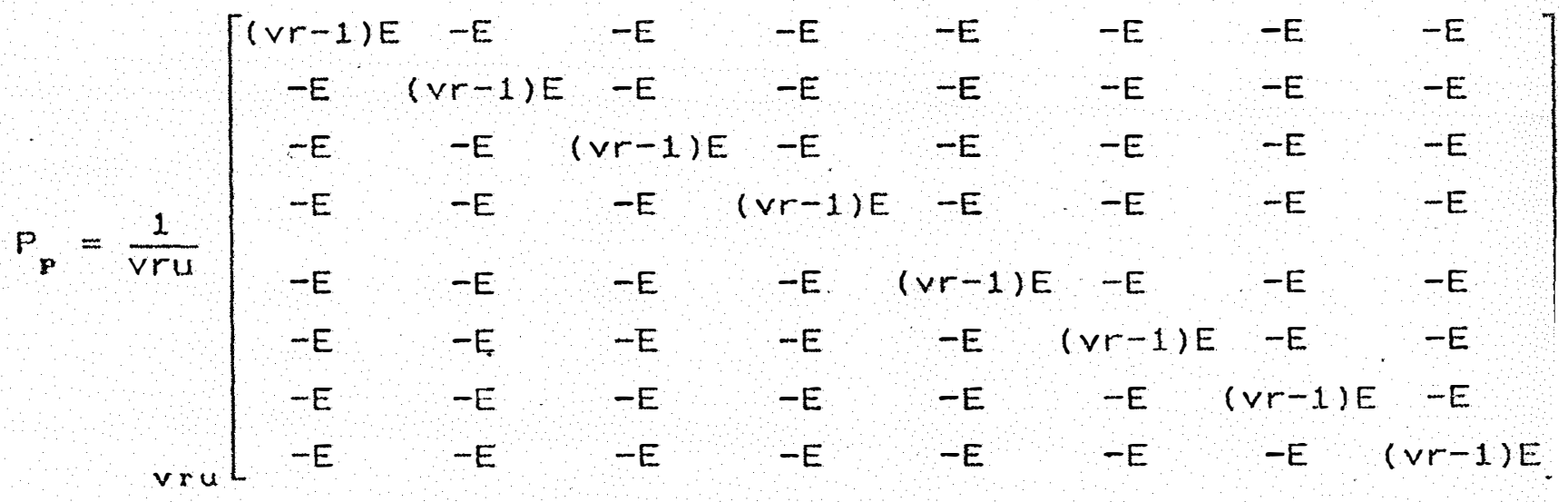

onde $E$ é uma submatriz de uns, de dimensöes ( $u \times u$ ). 
114.

APENDICE 2 - Um programa de análise

Aqui está apresentado um programa para ser utilizado no software NTIA, para analise intrablocos de um experimento em parcelas subdivididas com tratamentos principais em um PBIB. Através desse programa, pode-se fazer a análise do Exemplo 1, apresentado no item 4, a partir de um arquivo externo, editado por exemplo, pelo WS, com o nome de NOME.PRG.

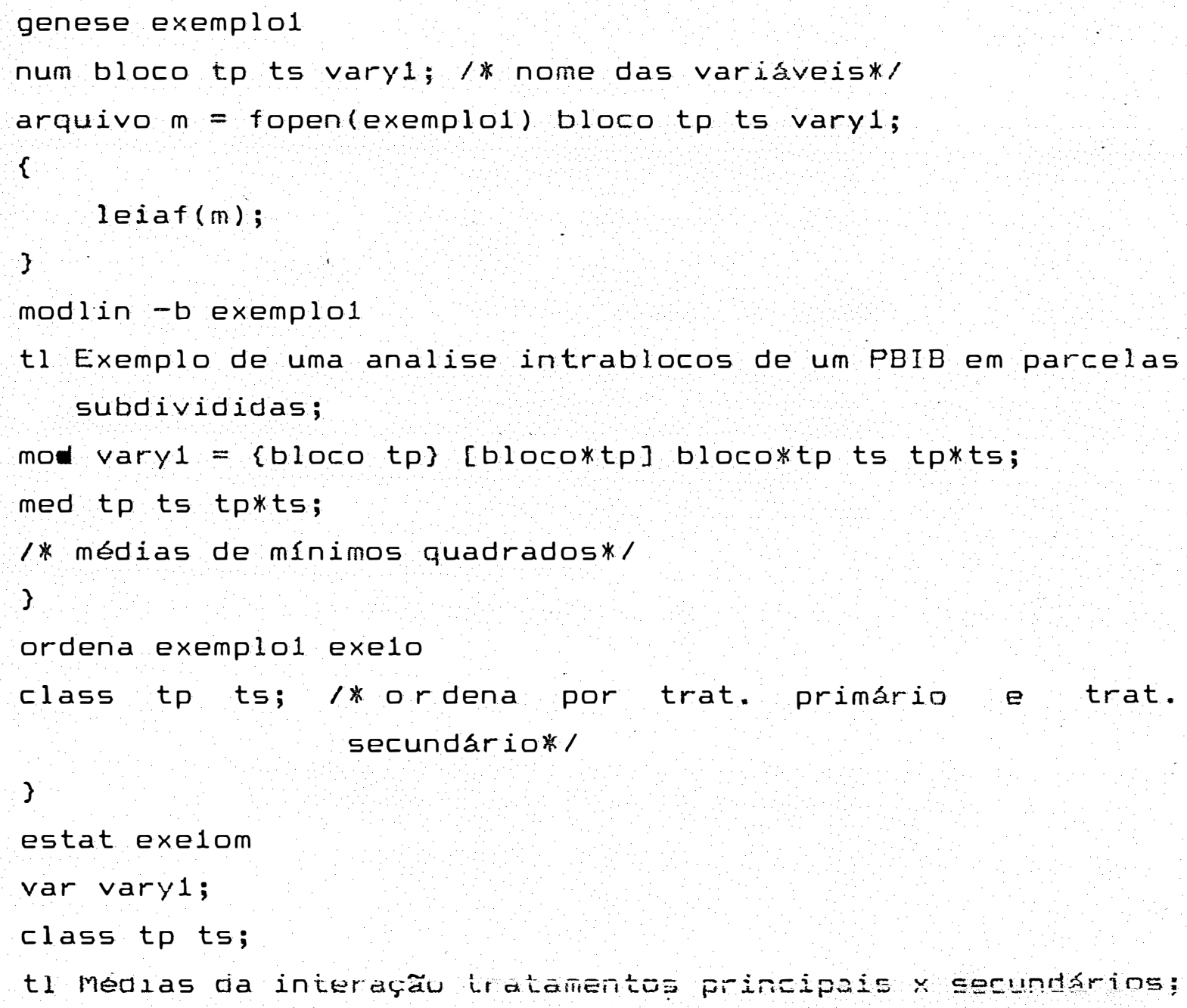




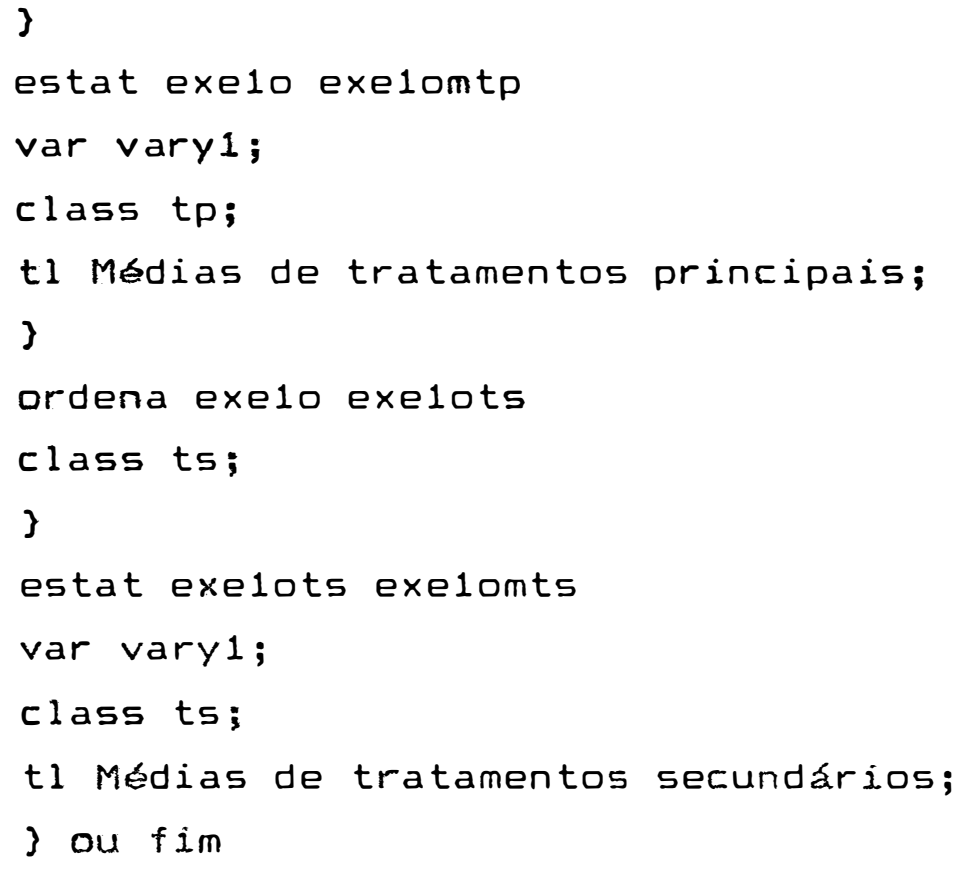

Sendo que:

(i) exemplol nome de um arquivo externo;

(ii) exe10, exelom, exelomtp, exelots, exelomts são arquivos criados pelo programa: dados ordenados, medias de interação, medias de tratamentos primários, dadas ordenados por tratamentos secundarios, medias de tratamentos secundarios, respectivamente.

Para executar o programa usa-se o modulo EXEC.

Exec nome.prg > exe1.sai 